\title{
NONPARAMETRIC UTILITY THEORY IN STRATEGIC SETTINGS: REVEALING PREFERENCES AND BELIEFS FROM PROPOSAL-RESPONSE GAMES
}

\author{
MARCO E. CASTILLO \\ Texas AEM University \\ PHILIP J. CROSS \\ AlixPartners \\ MIKHAIL FREER \\ ECARES, Université Libre de Bruxelles
}

\begin{abstract}
We explore the conditions under which behavior in a strategic setting can be rationalized as the best response to some belief about other players' behavior. We show that a restriction on preferences, which we term quasi-monotonicity, provides such a test for a family of ultimatum games. Preferences are quasi-monotone if an agent prefers an allocation that improves her payoff at least as much as that of others. In an experiment, we find that $94 \%$ of the proposers make choices that are arbitrarily close to quasi-monotone preferences and beliefs. We also find that $90 \%$ of the responders make inconsistent choices in no more than $5 \%$ of the decision problems. Subjects whose choices are consistent as proposers are also more likely to make consistent choices as responders and to believe that others act consistently. Finally, we find little support for the convexity of preferences.
\end{abstract}

JEL codes: C72, C78, C92

\section{INTRODUCTION}

Revealed preference analysis entails knowledge of the choice sets over which decisions are made. In strategic environments, the outcomes available to decision makers depend

E-mail addresses: (Castillo) marco.castillo@tamu.edu, (Cross) pcross@alixpartners.com, (Freer) mfreer@ulb.ac.be. 
on the decisions of other agents. Testing rational behavior in these contexts sometimes requires making strong assumptions about beliefs (see Sprumont 2000, Forges and Minelli 2009, Carvajal et al. 2013). In particular, these approaches test the joint hypothesis of rationality and equilibrium behavior. As Manski (2002, 2004) illustrates, decision rules cannot be identified separately from beliefs. In this paper, we show that, in a family of simple bargaining games, imposing a minimal set of restrictions on preferences and beliefs yields a test of a well-behaved preference ordering consistent with observed behavior. Our results show that properties of preferences can be identified without assuming equilibrium behavior.

In particular, we assume that the bargainers possess quasi-monotone preferences and that they believe that other bargainers also act according to preferences that are quasimonotone. The preferences of a bargainer are quasi-monotone if whenever the total surplus increases, she prefers allocations in which her payoff increases by more than other agents' payoffs. Note that this is a monotonicity notion; it does not imply that all other outcomes not included in the better-than set would be less preferred. The theory is agnostic about these alternative allocations. We also assume that the bargainers have preferences over lotteries that respect first-order stochastic dominance. Quasimonotonicity of preferences is akin to self-serving fairness. This behavior is consistent with the models of fairness in the literature (e.g., Fehr and Schmidt 1999, Bolton and Ockenfels 2000, and Charness and Rabin 2002), but these models are also consistent with other preferences. ${ }^{1}$

We study the implications of the quasi-monotonicity assumption together with optimizing behavior in simple bargaining games. In this context, a proposer's choice of an allocation is equivalent to a lottery that delivers her the chosen allocation or nothing. The setting implies that the probability of the allocation being implemented is equal to the proposer's (subjective) belief that the allocation will be accepted by the responder. We derive empirical implications when responders have quasi-monotone preferences and proposers' beliefs are consistent with this hypothesis. Our assumptions imply that, in the context of the ultimatum game, proposers must satisfy the Generalized Axiom of Revealed Preferences (GARP). The ultimatum game also provides a direct test of

\footnotetext{
${ }^{1}$ These models would imply stronger restrictions on preferences than quasi-monotonicity. We discuss this issue in Section 2. Quasi-monotonicity implies what Benjamin (2015) refers to as joint-monotonicity of preferences. Benjamin (2015) shows that joint-monotonicity of preferences is important in obtaining efficient outcomes in bilateral trade problems. Quasi-monotonicity also implies what Dufwenberg, Heidhues, Kirchsteiger, Riedel, and Sobel (2011) refer to as social monotonicity of preferences. They show that this property is necessary for obtaining Pareto optimal allocations in market economies with social preferences.
} 
the quasi-monotonicity of the preferences of responders. Responders do not face any uncertainty. Their behavior should therefore be consistent with maximization of a complete, transitive, and quasi-monotone preference ordering. Interestingly, behavior in the ultimatum game can also reveal (together with the previous assumptions) whether a responder has convex preferences. Consistency with quasi-monotonicity in both roles therefore provides a stronger test of our assumptions.

We test the theory by observing the choices of subjects in ultimatum games in laboratory experiments. Specifically, we observe subjects' bargaining behavior in a number of ultimatum games that differ in surplus size and the opportunity cost of dividing the surplus. This experimental design mimics real-world situations where buyers and sellers, each facing a different opportunity cost of money, bargain over the price of a non-divisible good. All subjects played both the proposer and the responder roles, which may have helped them to better understand the decision rules used by responders and get closer to optimal behavior.

We find that the behavior of the proposers is consistent with the quasi-monotonicity of their preferences and the belief that other agents behave as if they possess quasimonotone preferences. Sixty-nine of the 83 proposers (83\%) did not violate GARP and, of the 14 who did violate it, nine did so by only an arbitrarily small amount. ${ }^{2}$

Regarding the quasi-monotonicity of responders' preferences, we find that the behavior of 54 of the 83 subjects $(65 \%)$ is consistent with quasi-monotone preferences and that $90 \%$ of the responders make inconsistent choices in no more than $5 \%$ of the decision problems. The responders' behavior is heterogeneous: 31 subjects (37\%) accepted all offers in every game, and 41 subjects (49\%) rejected, on average, one or more offers per game. Twenty-three subjects $(28 \%)$ rejected three or more offers per game, on average. We find evidence against the convexity of responders' preferences. Fifty-two subjects (63\%) violated convexity, and 45 subjects (54\%) had at least six violations. All of the subjects who satisfied convexity as responders accepted all offers. Convexity was not common among the responders who did reject offers. Importantly, we find that subjects who satisfy responder rationality are also more likely to satisfy proposer rationality as well. However, we do not find a similar correlation between proposer rationality and convex responder rationality.

Our theory makes specific assumptions about the beliefs of the proposers. We test these assumptions using alternative belief elicitation techniques (see Section 4 and Appendix B). We find that $81 \%$ of the subjects' beliefs are consistent with responder

\footnotetext{
${ }^{2}$ That is, they have a critical cost to efficiency index (CCEI) (Afriat 1973) close to 1.
} 
rationality and $12 \%$ of the subjects' beliefs are consistent with convex responder rationality. The result is similar if we use incentivized belief elicitation techniques. In this case, we find that $75 \%$ percent of the subjects have beliefs consistent with responder rationality and $3 \%$ of the subjects have beliefs that are consistent with convex responder rationality. This result holds if we allow for a small measurement error ( 5 percentage points).

The strict convexity of preferences implies that a player in the role of a proposer behaves as if she has a preference for redistribution. Strict convexity rules out perfectly selfish preferences. Convexity, however, has additional implications for the behavior of players in the role of responders. Indeed, the convexity of preferences stands in contradiction with some models of fairness. Convexity neither implies nor is implied by models of inequality aversion, and it is therefore a logically independent hypothesis. ${ }^{3}$

In sum, we find that suitable relaxations of assumptions about preferences and beliefs can be used to derive testable implications of rational behavior in a strategic environment. Our experimental data support the assumptions we make. Moreover, we are able to replicate our results in two different populations. Our results can be extended to other proposal-response games under additional restrictions (e.g. reciprocal preferences ${ }^{4}$ ) using the same intuition as in Lemma 1 and Proposition 1 as well as to alternating-offer bargaining games.

The rest of the paper is organized as follows: Section 2 details our behavioral assumptions; Section 3 provides the testable implications of these assumptions in ultimatum games; Section 4 describes the experiment; Section 5 presents the experimental results; and Section 6 concludes the paper.

\section{THEORY}

2.1 Preferences Consider games in which players have preferences regarding their own monetary payoff and the monetary payoffs of other players. Let the vector of monetary payoffs in an $n$-player game be denoted by the $n$-vector $\mathbf{x} \equiv\left(x_{i}, \mathbf{x}_{-i}\right)$, where

\footnotetext{
${ }^{3}$ Suppose an agent in the role of responder has convex and quasi-monotone preferences. Hence, if she accepts an allocation $\left(x_{r}, x_{p}\right)=(2,1)$, she will also accept every allocation on the line that connects zero and this outcome. However, this is not necessarily true in Bolton and Ockenfels's (2000) model. Assume that the agent has the utility function $u\left(x_{r}, x_{p}\right)=x_{r}-\left|\frac{x_{r}}{x_{p}+x_{r}}-\frac{1}{2}\right|$, consistent with Bolton and Ockenfels's model. In this case, the utility of $(2,1)$ is $u(2,1)=5 / 6>0$, and the utility of $(2 / 10,1 / 10)$ is $-4 / 60<0$. That is, $\left(x_{r}, x_{p}\right)=(2 / 10,1 / 10)$, which is a convex combination of $(0,0)$ and $(2,1)$, will be rejected according to Bolton and Ockenfels's model, but it would be accepted according to convexity. Therefore, the inequality aversion model can generate data inconsistent with convex preferences.

${ }^{4}$ By reciprocal preferences we mean that responders choose allocations that are more favorable to proposers whenever the available choices allow higher payoffs to responders.
} 
$x_{i}$ is Player $i$ 's payoff and $\mathbf{x}_{-i}$ is the $n-1$ vector of payoffs for players other than Player $i$. The consumption set in an $n$-player game is $\mathbf{R}_{+}^{n}$.

The nature of the problem requires us to consider the set of binary lotteries. The lottery has two possible outcomes for $\mathbf{R}_{+}^{n}$ and the probability $q$ for the first outcome. Moreover, we fix the second outcome to be zero. Let $\mathcal{L}=\mathbf{R}_{+}^{n} \times[0,1]$ denote the set of binary lotteries, and let $(\mathbf{x}, q)=\mathbf{L} \in \mathcal{L}$ denote a binary lottery.

Let $\succeq_{i} \subseteq \mathcal{L} \times \mathcal{L}$ be the preference relation for Player $i$. Let $\succ_{i}$ denote the strict part of $\succeq_{i}$ and $\sim_{i}$ the indifferent part of $\succeq_{i}$. Throughout the analysis we maintain the following assumptions:

Completeness: For all $\mathbf{L}, \mathbf{L}^{\prime} \in \mathcal{L}$, either $\mathbf{L} \succeq_{i} \mathbf{L}^{\prime}$ or $\mathbf{L}^{\prime} \succeq_{i} \mathbf{L}$ or both.

Transitivity: For all $\mathbf{L}, \mathbf{L}^{\prime}, \mathbf{L}^{\prime \prime} \in \mathcal{L}$, if $\mathbf{L} \succeq_{i} \mathbf{L}^{\prime}$ and $\mathbf{L}^{\prime} \succeq_{i} \mathbf{L}^{\prime \prime}$, then $\mathbf{L} \succeq_{i} \mathbf{L}^{\prime \prime}$.

Stochastic Dominance Preference: For all $\left(\mathbf{x}^{\prime \prime}, 1\right) \succeq_{i}\left(\succ_{i}\right)\left(\mathbf{x}^{\prime}, 1\right)$ and $0 \leq p \leq$ $q \leq 1$,

$$
\left(\mathbf{x}^{\prime \prime}, q\right) \succeq_{i}\left(\succ_{i}\right)\left(\mathbf{x}^{\prime}, q\right) \succeq_{i}\left(\succ_{i}\right)\left(\mathbf{x}^{\prime}, p\right)
$$

Stochastic Dominance Preference has two aspects. First, Player $i$ prefers a lottery with a higher probability of a better outcome. Second, Player $i$ prefers a lottery with a better bundle (better bundles) if the probabilities are fixed.

Note that we are modeling the proposer's choice under uncertainty without the independence assumption of standard expected utility theory. ${ }^{5}$

Quasi-Monotonicity: For all $\left(x_{i}, \mathbf{x}_{-i}\right),\left(x_{i}^{\prime}, \mathbf{x}_{-i}^{\prime}\right) \in \mathbf{R}_{+}^{n}$,

$$
\left(x_{i}, \mathbf{x}_{-i}\right) \geq\left(x_{i}^{\prime}, \mathbf{x}_{-i}^{\prime}\right), \forall j \neq i x_{i}-x_{i}^{\prime} \geq x_{j}-x_{j}^{\prime} \Rightarrow\left(\left(x_{i}, \mathbf{x}_{-i}\right), q\right) \succeq_{i}\left(\left(x_{i}^{\prime}, \mathbf{x}_{-i}^{\prime}\right), q\right) .
$$

for every $q \in[0,1]$.

Strict Quasi-Monotonicity: For all $\left(x_{i}, \mathbf{x}_{-i}\right),\left(x_{i}^{\prime}, \mathbf{x}_{-i}^{\prime}\right) \in \mathbf{R}_{+}^{n}$,

$$
\left(x_{i}, \mathbf{x}_{-i}\right)>\left(x_{i}^{\prime}, \mathbf{x}_{-i}^{\prime}\right), \forall j \neq i x_{i}-x_{i}^{\prime} \geq x_{j}-x_{j}^{\prime} \Rightarrow\left(\left(x_{i}, \mathbf{x}_{-i}\right), q\right) \succ_{i}\left(\left(x_{i}^{\prime}, \mathbf{x}_{-i}^{\prime}\right), q\right) .
$$

for every $q \in[0,1]$.

Quasi-monotonicity (strict quasi-monotonicity) is a relaxation of the monotonicity (strict monotonicity) assumption from standard preference theory. That is, Player $i$

\footnotetext{
${ }^{5}$ The derivation of the expected utility property in the context of proposal-response games can be found in Gilboa and Schmeidler (2003).
} 
has quasi-monotone preferences if she prefers a bundle in which all players' payoffs are increased, but none by more than the increase in Player $i$ 's own payoff. Notice that, unlike the other properties, quasi-monotonicity is defined over the monetary outcomes and not over binary lotteries.

Convexity: For all $\mathbf{x} \in \mathbf{R}_{+}^{n}$ and $\alpha \in[0,1]$, if $\mathbf{x}^{\prime} \succsim_{i} \mathbf{x}$ and $\mathbf{x}^{\prime \prime} \succsim_{i} \mathbf{x}$, then $\alpha \boldsymbol{x}^{\prime}+(1-$ $\alpha) \mathbf{x}^{\prime \prime} \succsim_{i} \mathbf{x}$.

In the case of responders, the notion of rationality can be modified to include convexity of preferences. Convexity is not connected to the risk preferences of agents (responders) since responders face no risk. Instead, convexity refers to preferences for redistribution.

2.2 Beliefs In the context of proposal-response probability games, $q$ is endogenously determined. In particular, consider the situation in which Player $i$ offers Player $j$ the choice of implementing either bundle $\mathbf{x}$ or bundle zero. Note that, in this case, Player $j$ only has to have preferences over $\mathbf{R}_{+}^{n}$, because she does not face any uncertainty. In the notation above, this is a lottery $(\mathbf{x}, q)$ where $q$ is determined by Player $j .{ }^{6}$ The belief function $q: \mathbf{R}_{+}^{n} \rightarrow[0,1]$ is a continuous map of the proposed allocation into the proposer's subjective probability that $\mathbf{x}$ is realized. Let us state the restrictions on the belief function that we incorporate into the notion of the proposer's rationality.

Known Preference Restriction: For all $i$, Player $i$ knows that for all $j \neq i$, Player $j$ 's preferences over allocations are complete, transitive, continuous, and quasimonotone.

Belief Consistency: For all $\mathbf{x}, \mathbf{x}^{\prime}$, if for every $j \neq i \mathbf{x}^{\prime} \succeq_{j} \mathbf{x}$, then $q\left(\mathbf{x}^{\prime}\right) \geq q(\mathbf{x})$.

Belief Consistency states that if a proposer knows that bundle $\mathbf{x}^{\prime}$ is preferred to bundle $\mathbf{x}$ by all responders, ${ }^{7}$ then the proposer assigns a higher subjective probability to $\mathbf{x}^{\prime}$ rather than $\mathbf{x}$ being implemented. This is a rather weak assumption on its own and is restricted by the Known Preference Restriction. The latter implies that the proposer is guaranteed to have information about responders' preferences and expects them to act in accordance with responder rationality. Note that this does not imply that the proposer knows the entire preference relation of any responder.

\footnotetext{
${ }^{6}$ In the sense that $q$ is the probability of Player $j$ accepting allocation $\mathbf{x}$ in favor of allocation $\mathbf{x}^{\prime}$, according to Player $i$ 's belief.

${ }^{7}$ This allows generalization of some of the analysis to games with multiple responders who take actions simultaneously or sequentially.
} 
2.3 Two-player proposal-response games In what follows, we confine our attention to two-player games. If Player $i$ is the proposer, we denote $i$ as $\mathrm{p}$ and $j$ as $\mathrm{r}$, and if Player $i$ is the responder, we denote $i$ as $\mathrm{r}$ and $j$ as $\mathrm{p}$. In the ultimatum game, an allocation $\left(x_{\mathrm{p}}, x_{\mathrm{r}}\right)$ is chosen by the proposer from a given linear budget constraint, and the responder chooses either $\left(x_{\mathrm{p}}, x_{\mathrm{r}}\right)$ or $(0,0)$ as the realized allocation. For simplicity, we will refer to the lottery $\left(\left(x_{\mathrm{p}}, x_{\mathrm{r}}\right), q\left(\left(x_{\mathrm{p}}, x_{\mathrm{r}}\right)\right)\right) \in \mathcal{L}$ simply as $\left(x_{\mathrm{p}}, x_{\mathrm{r}}\right)$.

We use the term responder rationality to describe a subject with complete, transitive, and quasi-monotone preferences for allocations. We use the term proposer rationality to describe a subject with complete, transitive, continuous, strictly quasi-monotone preferences over binary lotteries who exhibits stochastic dominance and a continuous belief function that satisfies the known preference restriction and belief consistency properties. Further, we assume that every proposer exhibits proposer rationality and that every responder exhibits responder rationality. Following Debreu (1964), ${ }^{8}$ we can infer that proposer rationality implies the existence of a continuous utility function over binary lotteries $\left(U_{\mathrm{p}}\right)$ that represents the proposer's preferences.

Before proceeding with the formal results, we elaborate on the assumptions we have adopted. Proposer rationality requires non-satiation (over the space of certain outcomes). However, non-satiation in our context operates in the space of lotteries. We then require that proposers' preferences satisfy stochastic dominance and strict quasimonotonicity. This guarantees non-satiation over the space of lotteries, although we observe choices on the "projection" of the space of lotteries to the subspace defined by the belief function. In order to have empirical content, constraints on beliefs are necessary. To guarantee non-satiation of preferences (on the projection), we need to guarantee that for every allocation, there is a set of outcomes that are preferred by proposers and responders. The quasi-monotonicity of responders' preferences plays a dual role: (1) it provides information about responder preferences, and (2) it guarantees that for every allocation there are some allocations that are strictly preferred by the proposer and weakly preferred by the responder.

In the ultimatum game, ${ }^{9}$ we can obtain the following result:

Lemma 1. For any $\left(\left(x_{\mathrm{p}}, x_{\mathrm{r}}\right), q\left(x_{\mathrm{p}}, x_{\mathrm{r}}\right)\right) \in \mathcal{L}$ and any $a>0$, either $\left(\left(x_{\mathrm{p}}+a, x_{\mathrm{r}}+\right.\right.$ $\left.a), q\left(x_{\mathrm{p}}+a, x_{\mathrm{r}}+a\right)\right) \succ_{\mathrm{p}}\left(\left(x_{\mathrm{p}}, x_{\mathrm{r}}\right), q\left(x_{\mathrm{p}}, x_{\mathrm{r}}\right)\right)$ or $\left(\left(x_{\mathrm{p}}-a, x_{\mathrm{r}}-a\right), q\left(x_{\mathrm{p}}-a, x_{\mathrm{r}}-a\right)\right) \succ_{\mathrm{p}}$ $\left(\left(x_{\mathrm{p}}, x_{\mathrm{r}}\right), q\left(x_{\mathrm{p}}, x_{\mathrm{r}}\right)\right)$.

\footnotetext{
${ }^{8}$ The original result was stated in Debreu (1954), and a corrected proof is presented in Debreu (1964).

${ }^{9}$ Lemma 1 and Proposition 1 apply to $n$-player games, with one proposer and $n-1$ responders who make decisions in an arbitrary order.
} 
Proof. Recall that $\succeq_{\mathrm{p}}$ is complete. Therefore, at least one of the following assertions must be true: $(\mathbf{x}, q(\mathbf{x})) \succeq_{\mathrm{p}} 0$ or $(\mathbf{x}, q(\mathbf{x})) \preceq_{\mathrm{p}} 0$. We start by considering the first case. Note that $x_{\mathrm{r}}+a-x_{\mathrm{r}}=x_{\mathrm{p}}+a-x_{\mathrm{p}}$ and $\left(x_{\mathrm{p}}+a, x_{\mathrm{r}}+a\right)>\left(x_{\mathrm{p}}, x_{\mathrm{r}}\right)$. Then, by the quasimonotonicity of responders, $\left(\left(x_{\mathrm{p}}+a, x_{\mathrm{r}}+a\right), q\left(x_{\mathrm{p}}+a, x_{\mathrm{r}}+a\right)\right) \succeq_{\mathrm{r}}\left(\left(x_{\mathrm{p}}, x_{\mathrm{r}}\right), q\left(x_{\mathrm{p}}, x_{\mathrm{r}}\right)\right)$, and this is known by the proposer (using the known preference restriction) because it can be inferred from quasi-monotonicity alone. Then, by belief consistency, the following is true: $q\left(\left(x_{\mathrm{p}}+a, x_{\mathrm{r}}+a\right)\right) \geq q\left(\left(x_{\mathrm{p}}, x_{\mathrm{r}}\right)\right)$.

From stochastic dominance, we can infer that $\left(\left(x_{\mathrm{p}}+a, x_{\mathrm{r}}+a\right), q\left(\left(x_{\mathrm{p}}+a, x_{\mathrm{r}}+a\right)\right)\right) \succeq_{\mathrm{p}}$ $\left(\left(x_{\mathrm{p}}+a, x_{\mathrm{r}}+a\right), q\left(\left(x_{\mathrm{p}}, x_{\mathrm{r}}\right)\right)\right)$ and $\left(\left(x_{\mathrm{p}}+a, x_{\mathrm{r}}+a\right), q\left(\left(x_{\mathrm{p}}, x_{\mathrm{r}}\right)\right)\right) \succ_{\mathrm{p}}\left(\left(x_{\mathrm{p}}, x_{\mathrm{r}}\right), q\left(\left(x_{\mathrm{p}}, x_{\mathrm{r}}\right)\right)\right)$. Then, by transitivity ${ }^{10}$ and strict quasi-monotonicity, $\left(x_{\mathrm{p}}+a, x_{\mathrm{r}}+a\right) \succ_{\mathrm{p}}\left(x_{\mathrm{p}}, x_{\mathrm{r}}\right)$.

For the second case, $\left((\mathbf{x}, q(\mathbf{x})) \preceq_{\mathrm{p}} 0\right)$, similar reasoning can be used.

Lemma 1 states that the preferences of proposers exhibit non-satiation. Hence, proposers will have continuous and non-satiated utility functions.

\section{Testing the Theory}

Let $\mathbf{x}^{1}, \mathbf{x}^{2}, \ldots, \mathbf{x}^{T}$ be distinct allocations of payoffs, each lying on a linear budget constraint. Let $p^{1}, p^{2}, \ldots, p^{T}$ be the prices that define the linear budgets together with incomes $m^{1}, m^{2}, \ldots, m^{T}$. Following Varian (1992), we adopt the following two definitions: (i) $\mathbf{x}^{1}$ is directly revealed preferred to $\mathbf{x}^{2}$ if $\mathbf{x}^{2}$ is in the choice set when $\mathbf{x}^{1}$ is chosen; (ii) $\mathbf{x}^{1}$ is indirectly revealed preferred to $\mathbf{x}^{T}$ if $\mathbf{x}^{1}$ is directly revealed preferred to $\mathbf{x}^{2}$, which in turn is directly revealed preferred to $\mathbf{x}^{3}, \ldots$, which in turn is directly revealed preferred to $\mathbf{x}^{T}$; and (iii) $\mathbf{x}^{1}$ is strictly directly revealed preferred to $\mathbf{x}^{2}$ is in the interior of the choice set of $\mathbf{x}^{1}$. In the case of linear budgets, $\mathbf{x}^{1}$ is in the budget set of $\mathbf{x}^{2}$ if $p^{2} \mathbf{x}^{1} \leq p^{2} \mathbf{x}^{2}$, and $\mathbf{x}^{1}$ is in the interior of the choice set of $\mathbf{x}^{2}$ if $p^{2} \mathbf{x}^{1}<p^{2} \mathbf{x}^{2}$.

Generalized Axiom of Revealed Preference (GARP): If $\mathbf{x}$ is indirectly revealed preferred to $\mathbf{x}^{\prime}$, then $\mathbf{x}^{\prime}$ is not strictly directly revealed preferred to $\boldsymbol{x}$.

Figure 1 illustrates a violation of GARP in the case of a proposal-response game. Note that $\mathbf{x}$ is directly revealed preferred to $\mathbf{x}^{\prime}$ since it is in the budget of $(p, m)$. In addition, $\mathbf{x}$ is strictly within the budget of $\mathbf{x}^{\prime}$. Hence, there is a violation of GARP.

Theorem 1 (Afriat's (1967) Theorem). The following conditions are equivalent:

(i) There exists a non-satiated utility function that rationalizes the data.

(ii) The data satisfy GARP.

\footnotetext{
${ }^{10}$ If a preference relation is transitive and complete, then $\mathbf{x} \succeq_{i} \mathbf{x}^{\prime}$ and $\mathbf{x}^{\prime} \succ_{i} \mathbf{x}^{\prime \prime}$ imply that $\mathbf{x} \succ_{i} \mathbf{x}^{\prime \prime}$.
} 


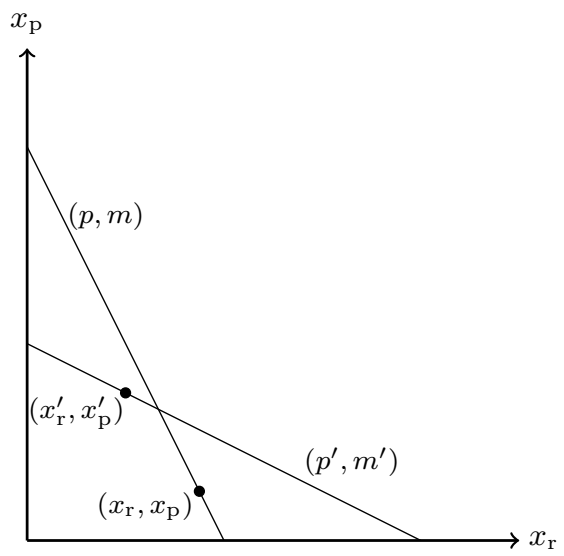

Figure 1. GARP in the Ultimatum Game

\subsection{Testing Proposer Rationality}

Proposition 1. In the ultimatum game, a proposer satisfying proposer rationality makes choices from linear budget sets that satisfy GARP. ${ }^{11}$

We prove Proposition 1 by applying Theorem 1 . However, the two statements operate in different spaces. The preference relation and the utility function in Theorem 1 are defined over $\mathbf{R}_{+}^{2}$, while the preference relation and the utility function in Proposition 1 are defined over $\mathcal{L}$. Hence, we use $R$ to denote the pseudo-preference relation such that for every $\mathbf{x}, \mathbf{x}^{\prime} \in \mathbf{R}_{+}^{2}, \mathbf{x} R \mathbf{x}^{\prime}$ if and only if $(\mathbf{x}, q(\mathbf{x})) \succeq_{\mathrm{p}}\left(\mathbf{x}^{\prime}, q\left(\mathbf{x}^{\prime}\right)\right)$. Therefore, it remains to show that $R$ is complete, transitive, continuous, and non-satiated.

Proof. The completeness, transitivity, and continuity of $R$ follow from the fact that $R$ is equivalent to the preference relation $\succeq_{\mathrm{p}}$ over a subset of $\mathcal{L}$. Hence, the completeness, transitivity, and continuity ${ }^{12}$ of $\succeq_{\mathrm{p}}$ imply the same properties for $R$.

The non-satiation of $R$ is implied by Lemma $1 .^{13}$ Therefore, $R$ is a complete, transitive, continuous, and non-satiated preference relation. Using Debreu's (1964) result, we can conclude that there is a continuous, non-satiated utility function that represents $R$. Hence, we conclude the proof by applying Theorem 1 .

\footnotetext{
${ }^{11}$ Note that the proposition can be generalized for monotone, compact, and balanced budgets, as in Forges and Minelli (2009). A balanced set is such that if $\mathbf{x} \in B$, then $\alpha x \in B$ for every $\alpha \in[0,1)$; Forges and Minelli (2009) call this property "Axiom H".

${ }^{12}$ To prove continuity, we appeal to the well-known result from general topology that a set is closed with respect to a subspace if and only if it can be represented as an intersection of some closed set with that subspace. Hence, if the upper and lower contour sets of $\succeq_{\mathrm{p}}$ are closed, then the contour sets of $R$ are also closed.

${ }^{13}$ Moreover, since every linear budget set contains the non-empty set of points that are strictly quasi-greater than zero, it can easily be shown that choices should lie on the boundary of the budget set.
} 
We make two remarks about Proposition 1. First, proposer rationality implies that the proposer's choices are consistent with GARP, but not vice versa. This happens because it is not possible to elicit (even with an infinite amount of experiments) the entire preference relation over $\mathcal{L}$. Hence, if choices over linear budgets satisfy GARP, then there is a non-satiated, continuous, complete, and transitive preference relation over a subset of $\mathcal{L}$.

Second, there are stronger assumptions than quasi-monotonicity (e.g., monotonicity) that imply the consistency of proposer behavior with GARP. ${ }^{14}$

3.2 Testing Responder Rationality In this section, we illustrate the consequences of different assumptions about preferences on responders' behavior. In proposal-response games, the responder chooses in a situation of certainty. The sole concern is the responder's preferences regarding allocations. In the ultimatum game, the responder chooses to accept or reject the proposed $\left(x_{\mathrm{p}}, x_{\mathrm{r}}\right)$ allocation. Thus, the responder's choice set is $\left\{\left(x_{\mathrm{p}}, x_{\mathrm{r}}\right),(0,0)\right\}$. A responder choosing from a sequence of distinct choice sets $\left\{\left(x_{\mathrm{p}}^{1}, x_{\mathrm{r}}^{1}\right),(0,0)\right\},\left\{\left(x_{\mathrm{p}}^{2}, x_{\mathrm{r}}^{2}\right),(0,0)\right\}, \ldots,\left\{\left(x_{\mathrm{p}}^{T}, x_{\mathrm{r}}^{T}\right),(0,0)\right\}$ can never violate GARP. The standard revealed preference axioms have no bite, although one can directly investigate the testable implications of the quasi-monotonicity assumption.

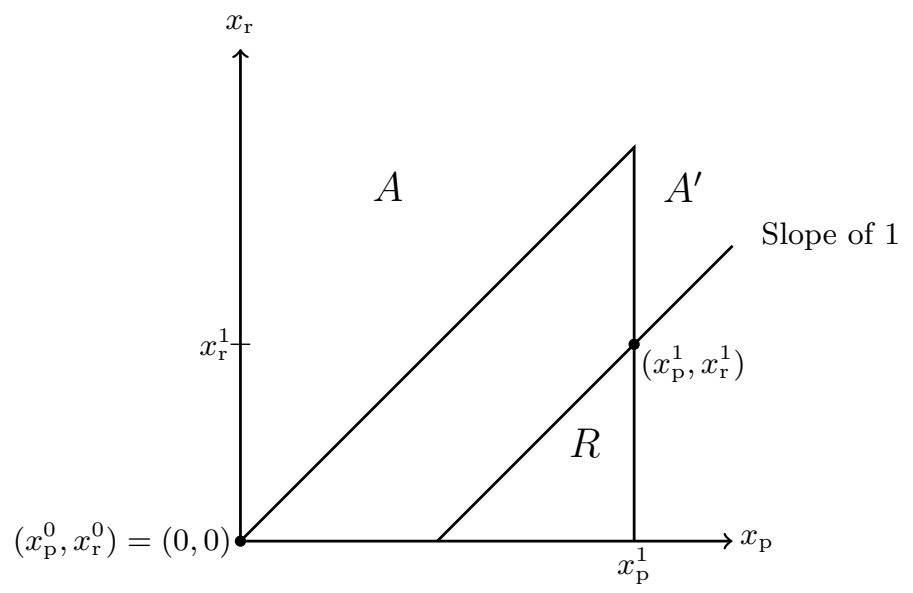

Figure 2. Testing Responder Rationality

Consider Figure 2, which illustrates a typical responder's choice set. The responder chooses either to accept the allocation $\mathrm{x}^{1}=\left(x_{\mathrm{p}}^{1}, x_{\mathrm{r}}^{1}\right)$ or to reject it in favor of the allocation $x^{0}=\left(x_{\mathrm{p}}^{0}, x_{\mathrm{r}}^{0}\right)=(0,0)$. By quasi-monotonicity, the responder should accept everything that lies above the $45^{\circ}$ line going through $(0,0)$; this set is labelled as $A$. Moreover, if the responder accepts $\mathbf{x}^{1}$, then by quasi-monotonicity, the responder would

\footnotetext{
${ }^{14}$ This is trivial, since the stronger condition would imply monotonicity and, therefore, the nonsatiation of the pseudo-preference relation $R$.
} 
also accept any proposed $\mathbf{x}$ that lies above the $45^{\circ}$ line going through $\mathbf{x}^{1}$ and is greater than $\mathbf{x}^{1}$ (the set $\left.A^{\prime}\right)$. Let $A=\left\{\mathbf{x} \geq \mathbf{0}, x_{\mathrm{r}} \geq x_{\mathrm{p}}\right\}$ denote the area above the $45^{\circ}$ line originating at zero. The acceptance area can be formally defined as follows:

$$
A^{t} \equiv\left\{\mathbf{x}: \mathbf{x}>\mathbf{x}^{t}, x_{\mathrm{r}} \geq x_{\mathrm{p}}-\left(x_{\mathrm{p}}^{t}-x_{\mathrm{r}}^{t}\right)\right\} \cup A
$$

Now suppose that the responder rejects $\mathbf{x}^{1}$, i.e., prefers $(0,0)$ to $\left(x_{\mathrm{p}}^{1}, x_{\mathrm{r}}^{1}\right)$. Note that by quasi-monotonicity, every $\mathbf{x}$ that lies in $R$ (below the $45^{\circ}$ line that goes through $\mathbf{x}^{1}$ ) is strictly less preferred than $\mathbf{x}^{1}$. Then, by transitivity, it is less preferred than $\mathbf{x}^{0}=(0,0)$. Hence, the responder should reject every bundle from the set $R$ if the responder rejects $\mathbf{x}^{1}$ :

$$
R^{t} \equiv\left\{\mathbf{x}: \mathbf{x} \leq \mathbf{x}^{t}, x_{\mathrm{r}} \leq x_{\mathrm{p}}-\left(x_{\mathrm{p}}^{t}-x_{\mathrm{r}}^{t}\right) \text { and } x_{\mathrm{p}} \leq x_{\mathrm{p}}^{t}\right\}
$$

Let $A_{x}$ denote the choice sets in which the responder accepted $\mathbf{x}^{1}$ and $R_{x}$ the choice sets in which the responder rejected $\mathrm{x}^{1}$ :

$$
\begin{gathered}
A_{x} \equiv\left\{t \in\{1, \ldots, T\}: \mathbf{x}^{t} \text { is accepted over }(0,0)\right\}, \\
R_{x} \equiv\left\{t \in\{1, \ldots, T\}: \mathbf{x}^{t} \text { is rejected in favor of }(0,0)\right\} .
\end{gathered}
$$

Proposition 2. The observed choices are made by a responder who satisfies responder rationality if and only if

$$
\left\{\mathbf{x}^{t}: t \in R_{x}\right\} \cap\left(A \cup\left(\bigcup_{t \in A_{x}} A^{t}\right)\right)=\emptyset
$$

The proof is provided in the Appendix. Note that the statement is equivalent to the existence of a complete, transitive, and quasi-monotone preference relation that generates the observed choices.

In this case, monotonicity has empirical content: if responders' preferences are monotone, then they would never reject $\mathbf{x}^{1}>(0,0)$. Note that, for responders, we can test the assumption of convexity of preferences.

Consider Figure 3, which illustrates a typical responder's choice set. Similar to the previous case, consider first the case in which the responder chooses $\mathbf{x}^{1}$ over $\mathbf{x}^{0}$. Convexity then implies that $\alpha \mathbf{x}^{1} \succeq_{\mathbf{r}} \mathbf{x}^{0}$ for any $\alpha \in[0,1]$. This, combined with quasimonotonicity, implies that the responder should accept every bundle from the set $A^{\prime \prime}$ (the area above the line that goes through $\mathrm{x}^{0}$ and $\mathrm{x}^{1}$ such that $x_{\mathrm{p}} \leq x_{\mathrm{p}}^{1}$ ) in addition to 


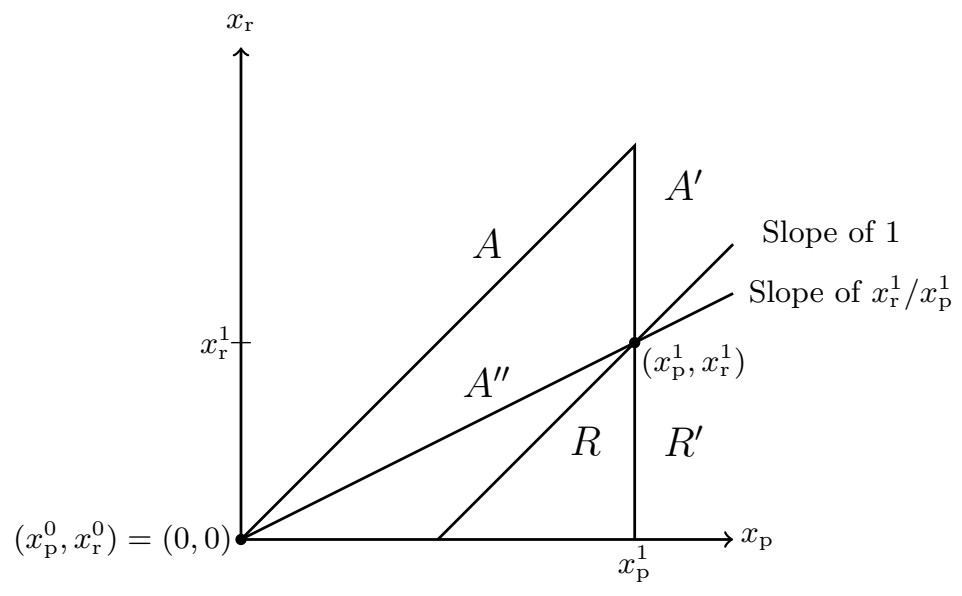

Figure 3. Testing Responder Convex Rationality

any bundle from $A$. The acceptance region under the assumption of convex responder rationality is then $A_{c}=A \cup A^{\prime} \cup A^{\prime \prime}$. Formally, $A_{c}^{t}$ can be defined as

$$
A_{c}^{t} \equiv A^{t} \cup\left\{\mathbf{x}: x_{\mathrm{p}} \leq x_{\mathrm{p}}^{t}, x_{\mathrm{r}} x_{\mathrm{p}}^{t} \geq x_{\mathrm{p}} x_{\mathrm{r}}^{t}\right\}
$$

If the responder rejects $\mathbf{x}^{1}$, then $\mathbf{x}^{0} \succ_{\mathrm{r}} \mathbf{x}^{1}$. Consider $\mathbf{x}=\gamma \mathbf{x}^{1}$ for $\gamma \in[1, \infty)$; if $\mathbf{x} \succeq_{\mathrm{r}} \mathbf{x}^{0}$, then, by convexity, $\mathbf{x}^{1} \succeq_{\mathrm{r}} \mathbf{x}^{0}$ because $\mathbf{x}^{1}$ can be represented as a convex combination of $\mathbf{x}^{0}$ and $\mathbf{x}$. This implies that every $\mathbf{x}$ from $R^{\prime}$ (the area below the line that goes through $\mathbf{x}^{0}$ and $\mathbf{x}^{1}$, such that $\mathbf{x}_{\mathrm{p}} \geq \mathbf{x}_{\mathrm{p}}^{1}$ ) should also be rejected. The rejection region under the assumption of convex responder rationality is then $R_{c}=R^{\prime} \cup R$. Formally, $R_{c}^{t}$ can be defined as

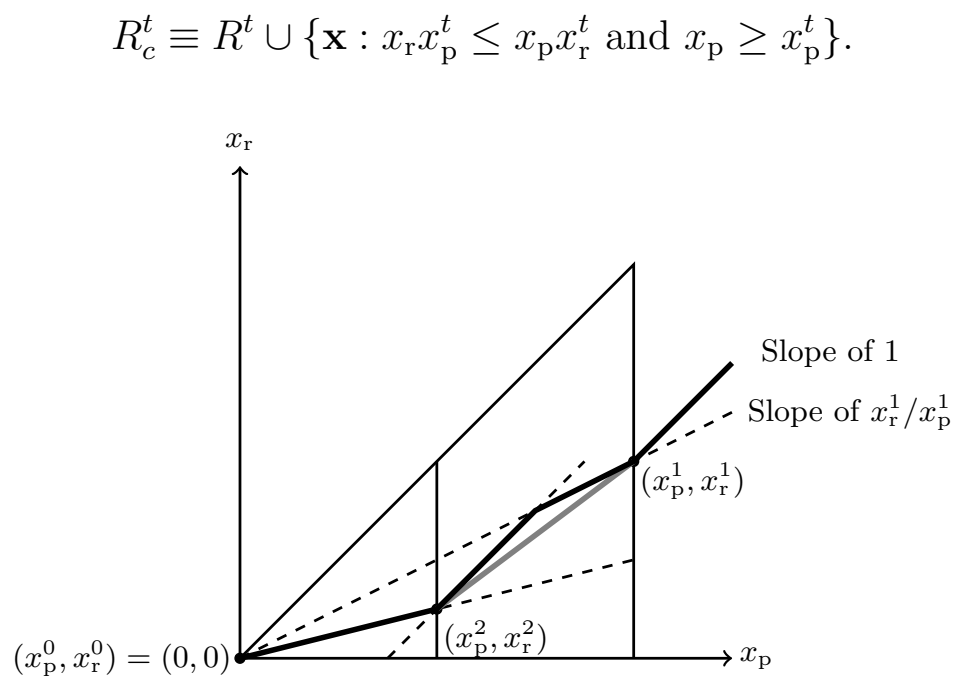

Figure 4. Necessity of Taking the Convex Hull

Note that to test responder convex rationality, it is not enough to consider only the union of acceptance areas generated by every accepted point. Figure 4 illustrates 
this point. In this case, $\mathbf{x}^{1}$ and $\mathbf{x}^{2}$ are both better than $\mathbf{x}^{0}$, and the union of the acceptance areas would just deliver the area above the thick line. Note that the union of the acceptance areas does not include the convex combinations of $\mathbf{x}^{1}$ and $\mathbf{x}^{2}$ (gray line), while by convexity we know that any convex combinations of $\mathrm{x}^{1}$ and $\mathrm{x}^{2}$ should be better than $\mathbf{x}^{0}$ and, as a result, should not be rejected. Therefore, to generate the acceptance area, we need to take the convex hull of the union of $A_{c}^{t}$. Let $C H(S)=\{x$ : $x=\sum_{y_{i} \in A \subseteq S} \alpha_{i} y_{i}$, with $\alpha_{i} \geq 0$ and $\left.\sum \alpha_{i}=1\right\}$ denote the convex hull of the set $S$.

The following refinement of Proposition 2 characterizes the empirical implications of convex responder rationality.

Proposition 3. The observed choices are made by a responder who satisfies convex responder rationality if and only if

$$
\left\{\mathrm{x}^{t}: t \in R_{x}\right\} \cap C H\left(A \cup\left(\bigcup_{t \in A_{x}} A_{c}^{t}\right)\right)=\emptyset
$$

The proof is in the Appendix.

The tests of proposer and responder rationality are different. First, they make decisions over different spaces. We can directly test the quasi-monotonicity of responders' preferences. This is not the case for proposers. For responders, we explicitly construct the sets of points that are better/worse than the choices available at each decision node. For proposers, linear budgets already include all the points that are quasi-smaller than a chosen point - this allows us to test the non-satiation/monotonicity of preferences without any additional construction.

Second, while we can determine the choice sets of proposers (by varying prices and income), we cannot determine the choice set faced by a responder. This choice set is determined by the actions of the proposer. To improve our knowledge of responders' preferences, we then use the strategy method.

\section{Experimental Design}

We implemented variations on the standard two-player ultimatum game employed by Guth et al. (1982) and Roth et al. (1991). The standard ultimatum game involves the proposer offering a division of $m$ dollars between the proposer $\left(x_{\mathrm{p}}\right)$ and the responder $\left(x_{\mathrm{r}}\right)$, so that $m=x_{\mathrm{p}}+x_{\mathrm{r}}$. The responder then accepts or rejects the offered $\left(x_{\mathrm{p}}, x_{\mathrm{r}}\right)$ allocation. If the responder accepts the offer, the responders' monetary payoff is $x_{\mathrm{r}}$ dollars and the proposer's monetary payoff is $x_{\mathrm{p}}$ dollars. If the responder rejects the offer, both players receive a monetary payoff of zero dollars.

Our experimental subjects play nine different ultimatum games with budgets $m=$ $x_{\mathrm{p}}+p x_{\mathrm{r}}$ with various endowments $(m)$ and relative prices of offers $(p)$. The subjects 
are volunteers from undergraduate economics courses. Each subject makes choices assuming both the role of the proposer and that of the responder in each of the nine games. There is a fifty-fifty chance of ultimately being assigned the role of proposer or responder, and an equal chance of each of the nine games being selected as the one whose choices determine the subjects' final payoffs. Proposers choose $x_{\mathrm{r}}$ from the linear budget constraint $m=x_{\mathrm{p}}+p x_{\mathrm{r}}$, discretized into 13 dollar allocations (almost all of which are integer values). These nine budgets are presented in Figure 5.

When assuming the responder role, subjects make their accept/reject decisions before they know which of the 13 allocations have been proposed. Consequently, for each of the 13 allocations, subjects make a choice to accept or reject it, thereby determining their response to whichever allocation is actually proposed. For example, for the ultimatum game with an endowment of $m=\$ 24$ and a relative price of giving of $p=1 / 3$, the choice sets for the proposer and the responder are

$$
\mathcal{C}=\{3,6,12,18,24,30,36,42,48,54,60,66,69\} \quad \text { and } \quad \mathcal{D}=\{0,1\}
$$

respectively. The proposer's and the responder's monetary payoffs as a function of $c \in \mathcal{C}$ and $d \in \mathcal{D}$ are $x_{\mathrm{p}}(c, d)=\left(24-\frac{1}{3} c\right) d$ and $x_{\mathrm{r}}(c, d)=c d$, respectively. The other eight versions of the ultimatum games are similarly defined.

For brevity, we summarize these games by convex, linear budget constraints (such as $\left.\$ 24=x_{\mathrm{p}}+\frac{1}{3} x_{\mathrm{r}}\right)$ rather than the actual discretized choice set $\mathcal{C}$. To make the choice sets more transparent, subjects were presented with the final dollar allocations rather than with budget constraints and endowments. ${ }^{15}$ Eighty-eight participants were recruited from undergraduate economics courses at Georgetown University. There were two experimental sessions, one with 43 participants and one with 45 participants. One participant in each session was chosen at random to be a monitor. The monitor made no decisions but verified to the other participants that the experimenters correctly followed the procedures described in the instructions. Once the participants were assembled, the instructions were read out loud, with participants reading along on their own copies. The subjects solved several preparatory exercises to familiarize themselves with the games, and the experimenter subsequently reviewed the correct answers. The subjects then proceeded to fill out the experimental decision forms, placing their completed decisions in a plain envelope. The nine games were randomly ordered on each subject's decision forms. However, the proposer and responder roles were presented systematically for each game, with the proposer decision always presented first.

\footnotetext{
${ }^{15}$ Appendix $\mathrm{C}$ displays the decision sheets that were used.
} 


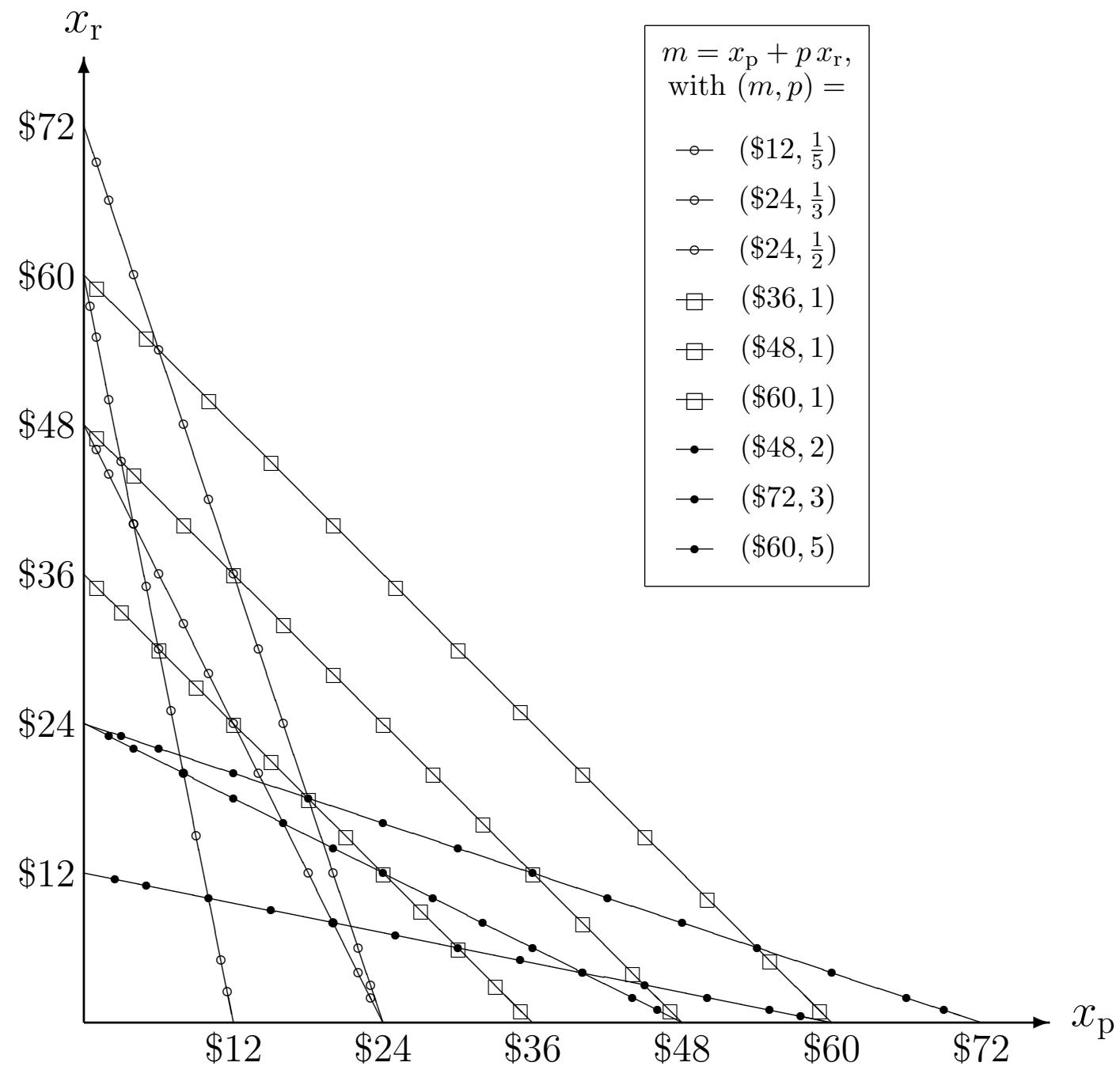

Figure 5. Budget Constraints Faced by Proposers

In plain view, these envelopes were collected, shuffled, and randomly separated into two equal-sized piles, one for proposers and one for responders. Once the proposerresponder pairs were formed, the forms were taken to a nearby room to calculate the payments. One of the nine games was chosen at random for each pair and implemented. The payments, along with an $\$ 8$ attendance reimbursement, were placed in a private envelope with only the subject's identification number on the outside. Another experimenter who was not involved in the calculation of payments handed out the envelopes to the participants, who were then escorted from the room. While payments were being calculated, subjects filled out a post-experiment questionnaire that elicited their understanding of the games, some expectation data, and some demographic covariates. The experiment lasted less than an hour, and the participants earned an average of $\$ 23.08$ (s.e. $\$ 1.70)$. Of the 86 participants, 55 were male and 31 were female. In addition to the two monitors, three subjects did not completely fill out their decision sheets. The analysis excludes them, leaving an experimental population of 83 subjects. 


\section{Results}

The observed choices of proposers and responders for each of the nine budgets are summarized in Table 1. Columns 2 and 3 give the means and standard deviations of the proposed $x_{\mathrm{r}}$ values. Column 4 shows the fraction of proposers who are generous, meaning their proposed $x_{\mathrm{r}}$ for a particular budget exceeded the minimum, and column 5 shows the mean proposals among the generous. The next four columns show the behavior of responders who adhered to a cutoff rule, meaning that for each budget there was a cutoff below which all proposed $x_{\mathrm{r}}$ values were rejected, and above which all proposed $x_{\mathrm{r}}$ values were accepted. Columns 6 and 7 show the mean and standard deviation of the highest rejected $x_{\mathrm{r}}$ values for each budget. Column 8 shows the fraction of rejectors - responders who rejected at least the minimum $x_{\mathrm{r}}$-and column 9 shows the mean of the highest rejected $x_{\mathrm{r}}$ values for the rejectors. The final column shows the number of responders who did not adhere to a cutoff rule for each budget. ${ }^{16}$

\begin{tabular}{|c|c|c|c|c|c|c|c|c|c|}
\hline \multirow{3}{*}{$\begin{array}{l}m=x_{\mathrm{p}}+p x_{\mathrm{r}} \\
\text { with }(m, p)=\end{array}$} & \multicolumn{4}{|c|}{ Proposed $x_{\mathrm{r}}$} & \multicolumn{4}{|c|}{ Highest rejected $x_{\mathrm{r}}$} & \multirow{3}{*}{$\begin{array}{l}\text { No cut- } \\
\frac{\text { off rule }}{\underline{n}}\end{array}$} \\
\hline & \multicolumn{2}{|c|}{ All proposers } & \multicolumn{2}{|c|}{ Generous } & \multicolumn{2}{|c|}{ All responders } & \multicolumn{2}{|c|}{ Rejectors } & \\
\hline & Mean & $\underline{\text { St.Dev }}$ & $\underline{\% \text {-age }}$ & $\underline{\text { Mean }}$ & Mean & $\underline{\text { St.Dev }}$ & $\underline{\% \text {-age }}$ & $\underline{\text { Mean }}$ & \\
\hline$\left(\$ 12, \frac{1}{5}\right)$ & $\$ 10.63$ & $\$ 9.27$ & $80.7 \%$ & $\$ 12.57$ & $\$ 4.00$ & $\$ 10.66$ & $47.0 \%$ & $\$ 8.53$ & 3 \\
\hline$\left(\$ 24, \frac{1}{3}\right)$ & $\$ 13.88$ & $\$ 9.55$ & $80.7 \%$ & $\$ 16.48$ & $\$ 5.10$ & $\$ 12.96$ & $45.8 \%$ & $\$ 11.13$ & 3 \\
\hline$\left(\$ 24, \frac{1}{2}\right)$ & $\$ 10.72$ & $\$ 6.31$ & $81.9 \%$ & $\$ 12.65$ & $\$ 4.51$ & $\$ 9.05$ & $44.6 \%$ & $\$ 10.11$ & 3 \\
\hline$(\$ 36,1)$ & $\$ 10.75$ & $\$ 6.87$ & $83.1 \%$ & $\$ 12.72$ & $\$ 4.25$ & $\$ 6.86$ & $51.8 \%$ & $\$ 8.21$ & 2 \\
\hline$(\$ 48,1)$ & $\$ 13.23$ & $\$ 8.72$ & $83.1 \%$ & $\$ 15.71$ & $\$ 5.23$ & $\$ 9.72$ & $51.8 \%$ & $\$ 10.09$ & 3 \\
\hline$(\$ 60,1)$ & $\$ 16.17$ & $\$ 11.37$ & $79.5 \%$ & $\$ 20.08$ & $\$ 6.39$ & $\$ 12.36$ & $56.6 \%$ & $\$ 11.28$ & 4 \\
\hline$(\$ 48,2)$ & $\$ 8.61$ & $\$ 5.84$ & $79.5 \%$ & $\$ 10.58$ & $\$ 4.00$ & $\$ 5.88$ & $53.0 \%$ & $\$ 7.54$ & 3 \\
\hline$(\$ 72,3)$ & $\$ 9.05$ & $\$ 6.23$ & $77.1 \%$ & $\$ 11.44$ & $\$ 4.02$ & $\$ 5.77$ & $50.6 \%$ & $\$ 7.95$ & 2 \\
\hline$(\$ 60,5)$ & $\$ 5.14$ & $\$ 3.63$ & $80.7 \%$ & $\$ 6.25$ & $\$ 2.81$ & $\$ 3.36$ & $59.0 \%$ & $\$ 4.76$ & 3 \\
\hline All 9 budgets & $\$ 10.91$ & $\$ 8.39$ & $80.7 \%$ & $\$ 13.18$ & $\$ 4.47$ & $\$ 9.05$ & $51.1 \%$ & $\$ 8.76$ & 5 \\
\hline
\end{tabular}

TABle 1. Summary of Proposer and Responder Behavior

The middle three rows of Table 1 show subjects' behavior in ultimatum games with budgets having a price of one and an income that increases from $\$ 36$ to $\$ 48$ to $\$ 60$. Examination of these three ultimatum games clearly revealed positive income effects. Mean proposals and the variance of proposals increased with income, as did the mean proposal among the generous. The mean and the variance of the highest rejected $x_{\mathrm{r}}$

\footnotetext{
${ }^{16}$ Table 1 shows that five responders made decisions from at least one budget that did not conform to a cutoff rule. The number of cutoff rule violations was nine for Subject 346, eight for Subject 416, seven for Subject 421 and one each for Subject 305 and Subject 443 .
} 
also increased with income, as did the mean highest rejected $x_{\mathrm{r}}$ among the rejectors. Compared to previous studies of unitary-price ultimatum games (Roth 1995; Camerer 2003), subjects in the present study made slightly smaller proposals on average.

Ultimatum games with $p \neq 1$ have previously been studied by Kagel, Kim, and Moser (1996) and Castillo and Cross (2008). Both of these studies collected data on ultimatum games with relative offer prices of $\frac{1}{3}$ and 3. In Kagel et al.'s (1995) study, the subjects played ten rounds assigned to either the proposer role or the responder role. Proposers offered $63.7 \%$ of their endowment from a $p=3$ budget and $24.2 \%$ from a $p=\frac{1}{3}$ budget, considerably higher than the corresponding shares in the one-shot ultimatum games studied here.

5.1 Proposer Rationality Were the revealed preference axioms violated by proposers, and if so, how severely? A useful measure of the severity of violations is Afriat's (1973) Critical Cost Efficiency Index ${ }^{17}$ (CCEI) (see Varian 1992). The CCEI is a relative measure - with the range $[0,1]$ - of how much one would have to relax each budget constraint to eliminate violations. The closer the CCEI is to one, the milder the relaxations of any budgets that are necessary to eliminate violations. A CCEI of 1 indicates no violations, and small violations are indicated by a CCEI of $1-\varepsilon{ }^{18}$ We refer to small violations as $\varepsilon$ violations and other violations as large violations. The upper panel of Table 2 shows the size distribution of the CCEI across proposers. Column 2 shows that 69 of the 83 proposers (83.1\%) did not violate GARP, and of the 14 violators, nine were $\varepsilon$ violators. No proposer had a CCEI of 0.80 or less.

How effective is GARP as a test of the hypothesis that proposers possess wellbehaved, quasi-monotonic preferences and believe responders' preferences to be likewise? Bronars' (1987) popular test compares this null hypothesis to the alternative that subjects make uniformly random choices from each budget - that is, $(a)$ the choice from each budget is the realization of a draw from a uniform distribution supported by that budget line, and $(b)$ choices from separate budgets are independent. The lower panel of Table 2 reports the power of Bronars' test from a simulation of 50,000 pseudosubjects. This power of $90 \%$ compares favorably to that computed in other studies (see Famulari 1995, Cox 1997, Sippel 1997, Harbaugh et al. 2001, and Andreoni and

\footnotetext{
${ }^{17}$ The first time analog of Critical Cost Efficiency for production analysis, called P-efficiency, was introduced by Afriat (1972) .

${ }^{18}$ That is, CCEI $>1-0.00001$. In some cases, the violations of GARP are arbitrarily close to one. This is the case if one of the chosen points lies within the intersection of the budget sets. Any $\varepsilon>0$ will eliminate the violation. Note that this type of violation can appear as a consequence of measurement error due to the discretization of the decision problem.
} 


\begin{tabular}{ccc}
\hline \hline $\begin{array}{c}\text { CCEI (Critical Cost } \\
\text { Efficiency Index) }\end{array}$ & Number of Subjects & Violations per Subject \\
\hline $1^{*}$ & 69 & 0 \\
$1-\varepsilon^{*}$ & 9 & 1.33 \\
{$\left[0.9,1-\varepsilon^{*}\right)$} & 3 & 2.67 \\
{$[0.8,0.9)$} & 2 & 2.50 \\
{$[0,0.8)$} & 0 & n.a. \\
\hline \hline
\end{tabular}

Power Analysis

Test

Test Power

Average Number

of Violation

\begin{tabular}{ccc}
\hline Bronars' Test & 0.9012 & 10.63 \\
e.d.f. test & 0.8136 & 7.42 \\
\hline \hline
\end{tabular}

* CCEI's of 1 and $1-\varepsilon$ denote no violations and small violations respectively

TABLE 2. Violations of Testable Implications for Proposers

Miller 2002). Indeed, we designed the experiment specifically to have a high Bronars' power - this is possible because Bronars' test is an ex ante test of rationality.

Alternatively, one can consider an ex post test of rationality where the alternative hypothesis supposes choices are independent draws from the empirical distribution function (e.d.f.) supported by each budget line - that is, the actual distribution of proposals observed in the experiment. Note that the power of this e.d.f. test is tied to observed behavior, and certain patterns of observed behavior can lead to the power being quite low. Consider the extreme case where no proposers ever make a generous offer; this yields an e.d.f. test with zero power. However, the pattern of proposals that was actually observed did not lead to an e.d.f. test with particularly low power. Column 2 in the lower panel of Table 2 shows that the e.d.f. test performed solidly in our experiment, having only a nine percentage point loss of power compared to Bronars' test.

5.2 Responder Rationality Table 3 presents the results of testing responder rationality and convex responder rationality using the empirical implications from Propositions 2 and 3. Column 3 shows that $65 \%$ of the subjects satisfied responder rationality and $90 \%$ of the subjects made no more than five violations. The benchmark of five mistakes is important because, formally, every subject faced 117 decision problems (13 options under nine different budget sets). Therefore, if the number of violations is no more than 5 , the subject makes mistakes in no more than $5 \%$ of the decision-making 
situations. Column 5 shows that only $37 \%$ of the subjects satisfied convex responder rationality and that $54 \%$ of the subjects made more than five mistakes, i.e., made mistakes frequently.

\begin{tabular}{ccccc}
\hline \hline \multirow{2}{*}{ Number of Violations } & \multicolumn{2}{c}{ Responder Rationality } & \multicolumn{2}{c}{ Convex Responder Rationality } \\
\cline { 2 - 5 } & Number of Subjects & Percent of Subjects & Number of Subjects & Percent of Subjects \\
\hline 0 & 54 & $65 \%$ & 31 & $37 \%$ \\
1 & 6 & $7 \%$ & 1 & $1 \%$ \\
2 & 8 & $10 \%$ & 3 & $4 \%$ \\
3 & 2 & $2 \%$ & 1 & $1 \%$ \\
4 & 4 & $5 \%$ & 1 & $1 \%$ \\
5 & 1 & $1 \%$ & 1 & $1 \%$ \\
$\geq 6$ & 8 & $10 \%$ & 45 & $54 \%$ \\
\hline \hline
\end{tabular}

\begin{tabular}{ccccc}
\hline \hline Power of Test & \multicolumn{2}{c}{ Proposer Rationality } & \multicolumn{2}{c}{ Convex Proposer Rationality } \\
\cline { 2 - 5 } Test & Power of Test & $\begin{array}{c}\text { Average Number } \\
\text { of Violations (std) }\end{array}$ & Power of Test & $\begin{array}{c}\text { Average Number } \\
\text { of Violations (std) }\end{array}$ \\
\cline { 2 - 5 } Random & 1.0000 & $47.0850(4.8914)$ & 1.0000 & $58.6110(5.3513)$ \\
Random Cutoff & 1.0000 & $34.1910(9.8979)$ & 1.0000 & $62.5970(11.1140)$ \\
e.d.f. & 1.0000 & $7.1360(2.4243)$ & 1.0000 & $16.3290(3.0731)$ \\
e.d.f. Cutoff & 0.9960 & $8.1660(3.8373)$ & 1.0000 & $24.0310(6.1289)$ \\
\hline \hline
\end{tabular}

TABLE 3. Violations of Testable Implications for Responders

It is worth noting that all 31 non-violators of convexity are among the 54 non-violators of responder rationality. We note that while responder rationality does not formally require a cutoff rule, convex responder rationality does. Furthemorer, these 31 nonviolators are subjects that accepted all offers in every game. Research by Andreoni, Castillo, and Petrie (2003) using the discrete and convex version of the ultimatum game showed that convexity for a fixed price and income is common. Our experiments were consistent with this finding as well. We found that only five of the 83 subjects violated a within-game cutoff rule. Hence, violations of convexity were not due to inconsistent responder behavior within a game, but rather inconsistent behavior across games.

To determine the power of the test, we generated 50,000 pseudo-subjects who followed one of the following rules. The first simple rule is an analog of Bronars' test in which a pseudo-subject is equally likely to accept or reject any given alternative. Second, we considered adding a cutoff rule to Bronars' test-each pseudo-subject followed a cutoff rule that was chosen at random separately for each game. The third test was an e.d.f. test in which every pseudo-subject accepted an offer according to the empirical distribution of acceptances for such an offer. Finally, we randomly assigned cutoff rules according to their empirical distribution. Note that the power of all tests was almost 1, i.e., none of the pseudo-subjects showed behavior that was consistent with the notions 
of rationality, and the mean number of violations was significantly higher than the mean number of violations for the real subjects (2.37 for responder rationality and 16.08 for convex responder rationality). This enabled us to conclude that the test we conducted has enough power to guarantee that our subjects' choices are actually consistent with the notions of rationality and that the observed results are not false positives.

\begin{tabular}{ccc}
\hline \hline & $\begin{array}{c}\text { Consistent with } \\
\text { Responder Rationality }\end{array}$ & $\begin{array}{c}\text { Inconsistent with } \\
\text { Responder Rationality }\end{array}$ \\
\cline { 2 - 3 } Consistent with Proposer Rationality $(C C E I=1)$ & $48(58 \%)$ & $21(25 \%)$ \\
Inconsistent with Proposer Rationality $(C C E I \neq 1)$ & $6(7 \%)$ & $8(10 \%)$ \\
\hline \hline
\end{tabular}

TABLE 4. Cross Table: Proposer Rationality and Responder Rationality

Table 4 compares subjects' consistency with quasi-monotonicity as proposers and responders. The majority of the subjects (58\%) satisfied quasi-monotonicity as proposers and responders. Quasi-monotone proposers were more likely to be quasi-monotone responders than non-quasi-monotone proposers (70\% v. 43\%, Fisher's exact test p-value $=$ 0.070). Note, however, that a sizable proportion of the subjects (25\%) satisfied quasimonotonicity as proposers, but not as responders. Table 4 provides support for the Known Preference Restriction assumption. Only 11\% (6 out of 54 subjects) of the subjects that were quasi-monotone as responders failed quasi-monotonicity of preferences and beliefs as proposers.

\begin{tabular}{ccc}
\hline \hline & $\begin{array}{c}\text { Consistent with Convex } \\
\text { Responder Rationality }\end{array}$ & $\begin{array}{c}\text { Inconsistent with Convex } \\
\text { Responder Rationality }\end{array}$ \\
\cline { 2 - 3 } Consistent with Proposer Rationality $(C C E I=1)$ & $30(36 \%)$ & $39(47 \%)$ \\
Inconsistent with Proposer Rationality $(C C E I \neq 1)$ & $1(1 \%)$ & $13(16 \%)$ \\
\hline \hline
\end{tabular}

TABle 5. Cross Table: Proposer Rationality and Convex Responder Rationality

Table 5 compares subjects' consistency with quasi-monotonicity as proposers and convexity as responders. The choices of the majority of the subjects $(63 \%)$ were not consistent with convexity of preferences. Quasi-monotone proposers were more likely to satisfy convexity as responders than non-quasi-monotone proposers $(45 \%$ v. $7 \%$, Fisher's exact test p-value $=0.013$ ). Note, however, that the only subjects whose choices as responders satisfied convexity were subjects who never rejected offers. This calls into doubt the assumption of the convexity of preferences in models of responders' behavior.

5.3 Beliefs We collected subjects' expectations after the experiment was completed, while payments were being prepared. Subjects were asked to provide an estimate of the 
probability that a particular offer would be rejected had it been offered by a responder. In particular, subjects were asked to answer questions of the form:

What do you think is the percent chance that Proposal Rule a would be Rejected by the Responder? $\bigcirc 0 \% \bigcirc 1 \%-30 \% \bigcirc 31 \%-70 \% \bigcirc 71 \%-99 \% \bigcirc 100 \%$.

This procedure is suggested by Manski (2004), and Manski and Neri (2013) implemented an incentivized version to elicit second-order beliefs in strategic games. A distinct advantage of this procedure is that it allows subjects to express uncertainty about their beliefs. Most of the literature on belief elicitation is devoted to the elicitation of point probabilistic beliefs (see Schotter and Treviño (2014) for a thorough discussion on the elicitation of beliefs). A potential drawback is that the elicitation is not incentivized. However, we show in Appendix B that use of Hossain and Okui's (2013) incentivized belief elicitation task produces similar results. Just as important, Appendix B also provides a replication of our original choice experimental results.

Table 6 reports the distribution of answers for all of the allocation rules we asked. We observe that subjects reported that allocations that are less favorable to responders are more likely to be rejected.

Table 7 reports whether the elicited beliefs are consistent with our assumptions of Known Preference Restrictions and Belief Consistency. These hypotheses cannot be tested separately; therefore, we evaluate them jointly. ${ }^{19}$

We now describe how we tested for belief consistency. Belief consistency implies that if allocation $\mathbf{x}$ is preferred to $\mathbf{x}^{\prime}$ by all proposers, then the probability that $\mathbf{x}$ is rejected should be no greater than the probability of rejecting $\mathbf{x}^{\prime}$. Additional restrictions are implied by the Known Preference Restriction. Let $b^{t}$ denote the belief that $\mathbf{x}^{t}$ is rejected. If beliefs are consistent with responder rationality, then if outcome $\mathbf{x}^{t}$ is greater than $\mathbf{x}^{s}$ according to quasi-monotonicity, the probability of rejecting $\mathbf{x}^{s}$ should be greater than the probability of rejecting $\mathbf{x}^{t}$.

Corollary 1. Let $A=\left\{\mathbf{x} \geq \mathbf{0}, x_{\mathrm{r}} \geq x_{\mathrm{p}}\right\}$ and $A^{t} \equiv\left\{\mathbf{x}: \mathbf{x}>\mathbf{x}^{t}, x_{\mathrm{r}} \geq x_{\mathrm{p}}-\left(x_{\mathrm{p}}^{t}-x_{\mathrm{r}}^{t}\right)\right\} \cup A$. $A$ set of beliefs $b^{1}, \ldots, b^{T}$ is consistent with responder rationality if and only if for every $\mathbf{x}^{s} \in A^{t} \backslash A, b^{s} \leq b^{t}$.

We now present a test of whether beliefs are consistent with (convex) responder rationality. Recall that subjects are randomly matched to a member of the population

\footnotetext{
${ }^{19}$ Each assumption taken alone does not have empirical content. If a player knows that other players are rational, but does not update beliefs correspondingly, then she still satisfies the Known Preference Restriction assumption. If a player has beliefs consistent with alternative preferences/notions of rationality, these beliefs do not have to be consistent with the tests we propose.
} 


\begin{tabular}{cccccc}
\hline \hline Allocation & \multicolumn{5}{c}{ Probability $q(x)$ that offer $x$ will be rejected: } \\
\cline { 2 - 6 }$\left(x_{\mathrm{p}}, x_{\mathrm{r}}\right)$ & $=0$ & $\in[1,30]$ & $\in[31,70]$ & $\in[71,99]$ & $=100$ \\
\hline$(23,3)$ & 9.6 & 26.5 & 21.7 & 36.1 & 6.0 \\
$(22,6)$ & 12.0 & 36.1 & 32.5 & 19.3 & 0.0 \\
$(20,12)$ & 30.1 & 55.4 & 12.0 & 2.4 & 0.0 \\
$(18,18)$ & 72.3 & 26.5 & 0.0 & 1.2 & 0.0 \\
\hline$(59,1)$ & 9.6 & 19.3 & 14.5 & 43.4 & 13.3 \\
$(50,10)$ & 20.5 & 28.9 & 28.9 & 19.3 & 2.4 \\
$(40,20)$ & 27.7 & 47.0 & 21.7 & 3.6 & 0.0 \\
$(30,30)$ & 72.3 & 26.5 & 1.2 & 0.0 & 0.0 \\
\hline$(60,4)$ & 9.6 & 22.9 & 16.9 & 44.6 & 6.0 \\
$(40,8)$ & 14.5 & 28.9 & 32.5 & 22.9 & 1.2 \\
$(36,12)$ & 27.7 & 42.2 & 22.9 & 7.2 & 0.0 \\
$(18,18)$ & 71.1 & 28.9 & 0.0 & 0.0 & 0.0 \\
\hline$(55,1)$ & 9.6 & 20.5 & 10.8 & 42.2 & 16.9 \\
$(40,4)$ & 12.0 & 27.7 & 21.7 & 34.9 & 3.6 \\
$(25,7)$ & 20.5 & 39.8 & 24.1 & 15.7 & 0.0 \\
$(10,10)$ & 68.7 & 30.1 & 1.2 & 0.0 & 0.0 \\
\hline \hline
\end{tabular}

TABLE 6. Distribution of Elicited Beliefs

of responders. Beliefs are said to be consistent with (convex) responder rationality if there is a population of (convex) responder rational players whose probability of rejecting the outcome $\mathbf{x}^{t}$ is equal to the proposer's belief. Recall that the convex acceptance region is defined as $A_{c}^{t} \equiv A^{t} \cup\left\{\mathbf{x}: x_{\mathrm{p}} \leq x_{\mathrm{p}}^{t}, x_{\mathrm{r}} x_{\mathrm{p}}^{t} \geq x_{\mathrm{p}} x_{\mathrm{r}}^{t}\right\}$ (see Figure 3). That is, if $\mathbf{x}^{t}$ is accepted, then every convex combination of zero and $\mathbf{x}^{t}$ is better than zero and therefore should be accepted. Hence, every allocation on the line that connects zero and $\mathbf{x}^{t}$ should be accepted. Moreover, quasi-monotonicity implies that every point above this line should also be accepted. Furthermore, if we consider a population of players with convex preferences, then the probability of rejection of every point in the convex acceptance region of $\mathbf{x}^{t}$ should be no greater than the probability of rejection of $\mathbf{x}^{t}$. However, the convex acceptance region guarantees that for every point, the upper contour set is convex if that point was the only accepted one. If more than one point was accepted, then the union of the convex acceptance regions may not itself be convex (see Figure 4). Therefore, it is necessary to take the convex hull of the union of the convex acceptance regions. Let $C=\left\{\mathbf{x}^{t}: t \in\{1, \ldots, T\}\right\}$ denote the set of all points over which beliefs are elicited, and let $2^{C}$ denote the power set of $C$. 
Corollary 2. A set of beliefs $b^{1}, \ldots, b^{T}$ is consistent with convex responder rationality if and only if $x^{s} \in C H\left(A \cup\left(\bigcup_{x^{t} \in S} A_{c}^{t}\right)\right)$ implies $b^{s} \leq \max _{x^{t} \in S}\left\{b^{t}\right\}$ for every $S \in 2^{X \backslash\left\{x^{s}\right\}} .^{20}$

Corollaries 1 and 2 are statements about point beliefs, while the beliefs we elicited are interval beliefs. We therefore test for the existence of point beliefs that lie within the elicited range provided to subjects and that satisfy the belief consistency criteria.

\begin{tabular}{|c|c|c|}
\hline & $\begin{array}{l}\text { Beliefs Consistent with } \\
\text { Responder Rationality }\end{array}$ & $\begin{array}{c}\text { Beliefs Consistent with } \\
\text { Convex Responder Rationality }\end{array}$ \\
\hline All Subjects & $67(83)$ & $10(83)$ \\
\hline Satisfy Proposer Rationality & $61(69)$ & $10(69)$ \\
\hline $\begin{array}{l}\text { Satisfy Proposer and } \\
\text { Responder Rationality }\end{array}$ & $43(48)$ & $9(48)$ \\
\hline $\begin{array}{c}\text { Satisfy Proposer and } \\
\text { Convex Responder Rationality }\end{array}$ & $25(30)$ & $9(30)$ \\
\hline \multicolumn{3}{|c|}{ * Total number of subjects falling in the corresponding category given in parenthesis } \\
\hline \multicolumn{3}{|l|}{ Power of Test } \\
\hline Random & 0.9970 & 1.0000 \\
\hline e.d.f & 0.9100 & 1.0000 \\
\hline
\end{tabular}

TABLE 7. Consistency of Beliefs

Table 7 reports the degree of consistency of individual beliefs with the assumptions of this paper. The first column includes subjects who believe that responders have quasi-monotone preferences, and the second column includes subjects who believe that responders have convex preferences. Each row in Table 7 presents results according to the characteristic of the subject reporting the beliefs. We can see that $67(81 \%)$ of all subjects have beliefs that are consistent with responder rationality. If we consider only subjects whose beliefs are consistent with proposer rationality, we observe that 61 $(88 \%)$ of these subjects have beliefs consistent with responder rationality. Regarding beliefs that are consistent with responders having convex preferences, we see that only $10(12 \%)$ of all the subjects have beliefs consistent with the notion of convex responder rationality. Moreover, five of these subjects assigned the same belief to every allocation.

To test the robustness of these results, we performed power tests for the consistency of the beliefs with responder rationality. We found that at least $91 \%$ of the random subjects had beliefs that fail the test, and $100 \%$ of the random subjects had beliefs that are not consistent with the notion of convex responder rationality. In sum, the elicited

\footnotetext{
${ }^{20}$ Although the precise test is NP-hard, the number of sets one should consider is $2^{T-1}$ for every $T$ observations. Moreover, at every step, it is necessary to take a convex hull of the set of points.
} 
beliefs were consistent with the assumptions we made and the analysis of the choice data we provided.

In our experimental design, we asked every subject to complete three tasks: to make decisions as proposers and as responders and to report beliefs. This allows us to identify which assumptions of proposer rationality failed. We observe that $43(52 \%)$ subjects passed all three tests, 5 (6\%) failed only consistency of beliefs, and 2 (2\%) failed only proposer rationality (i.e., violated stochastic dominance). In addition, 21 of the subjects' beliefs are consistent with proposer rationality but are not consistent with responder rationality, and 18 of the subjects passed the belief consistency test. These subjects seem to have consistent beliefs and behave rationally, but fail to have quasi-monotone preferences themselves. $^{21}$ The empirical analysis of beliefs presented so far relies on hypothetical questions. A more robust test of our assumptions would require obtaining similar results using incentivized elicitation of beliefs. We therefore collected additional experimental data on choices and beliefs using incentivized methods (Hossain and Okui, 2013). Table B.6 presents the results on consistency of beliefs using this new data set, and Table B.1 in the appendix summarizes the choice data. Tables B.2 and B.3 show that the new experimental sessions mimic the results in Tables 2 and 3 in the paper. Further, Table B.6 shows that our results regarding beliefs mimic those using nonincentivized methods. The similarities are even clearer if we relax the test of consistency to allow for a 5 percentage point measurement error. This provides further support for our assumptions and original results.

\section{Conclusions}

Samuelson (1938) revealed that a preference approach provides an intuitive and powerful way to test the empirical content of microeconomic theory. The usefulness of this approach has been apparent in the many applications and extensions over the years. ${ }^{22}$

\footnotetext{
${ }^{21}$ Formally, various monotonicity assumptions imply behavior consistent with GARP. For instance, subjects might prefer outcomes that increase proposers' and responders' payoffs equally. This, together with the quasi-monotonicity of responders' preferences, would imply behavior consistent with GARP, but it would lead to a rejection of responder rationality.

${ }^{22}$ Revealed preference analyses have been used to study preferences for giving and social preferences (Andreoni and Miller 2002; Fisman, Kariv and Markovits 2007), psychiatric patients (Battalio et al. 1973), children (Harbaugh, Krause, and Berry 2001), rats, pigeons, and monkeys (Kagel, Battalio, and Green 1995; Chen, Lakshminarayanan, and Santos 2006), risk preferences (Choi et al. 2007; Andreoni and Harbaugh 2009), characteristic models (Blow, Browning, and Crawford 2008), household bargaining (Cherchye, De Rock, and Vermeulen 2007), rational expectations (Browing 1989), habits (Crawford 2010), market equilibriums (Brown and Matzkin 1996), decisions on nonlinear budget sets (Matzkin 1991; Forges and Minelli 2009; Chavas and Cox 1993), and games (Sprumont 2000).
} 
This paper investigates the revealed preference approach in strategic environments. We show that a completely nonparametric analysis of a simple game is informative. In doing so, we have identified basic restrictions that must be placed on behavior to be consistent with this approach. We observed the behavior of bargainers in a number of ultimatum games and found that the behavior strongly supports the assumption of quasi-monotone preferences. This implies that, even absent parametric assumptions about preferences or collecting data on beliefs, we can extrapolate behavior to counterfactual games. Importantly, though, the measures of belief are strongly consistent with our assumptions.

Quasi-monotonicity is consistent with many models of fairness (Fehr and Schmidt 1999; Bolton and Ockenfels 2000; and Charness and Rabin 2002). ${ }^{23}$ Interestingly, our approach also suggests that further assumptions in models of fairness, such as homotheticity or quasi-linearity, are testable nonparametrically. In this context, we also find evidence against the convexity of preferences.

Additional assumptions beyond quasi-monotonicity are needed to rationalize behavior in other games (e.g., the investment game, as shown in Berg, Dickhaut, and McCabe 1995). Our study illustrates that the revealed preference approach provides a framework to systematically study these assumptions.

\footnotetext{
${ }^{23}$ Agents with quasi-monotone preferences will never reject offers that are favorable to them, while some models of fairness might allow this to occur.
} 


\section{Appendix A: Proofs}

To prove the propositions, we need to introduce additional notation. First, we use set-theoretic notation for the preference relations. A set $R \subseteq X \times X$ is said to be a preference relation. We denote the set of all preference relations on $X$ as $\mathcal{R}$. We denote the reverse preference relation as $R^{-1}=\{(x, y) \mid(y, x) \in R\}\left(P^{-1}(R)=\right.$ $\{(x, y) \mid(y, x) \in P(R)\})$. The symmetric part of $R$ is $I(R)=R \cap R^{-1}$, and the asymmetric part is $P(R)=R \backslash I(R)$. We denote the non-comparable part of $R$ as $N(R)=X \times X \backslash$ $\left(R \cup R^{-1}\right)$. We say that relation $R^{\prime}$ is an extension of $R$, denoted as $R \preceq R^{\prime}$, if $R \subseteq R^{\prime}$ and $P(R) \subseteq P\left(R^{\prime}\right)$. Note that for every $R \subseteq R^{\prime}, R^{\prime}$ is an extension of $R$ if and only if $P^{-1}(R) \cap R=\emptyset$ (for the proof see Demuynck (2009)). Hereafter, we will refer to this property as consistency with $R^{\prime}$.

Next, we formally define the revealed preference relation. Let $\left(x^{t}, B^{t}\right)_{t=1 \ldots T}$ be a finite consumption experiment in which $x^{t}$ are the chosen points and $B^{t}$ are budgets. We assume that all budgets are compact and monotone. ${ }^{24}$ Let $R_{E}$ denote the revealed preference relation. $\left(x^{t}, y\right) \in R_{E}$ if $y \in B^{t},\left(x^{t}, x^{t}\right) \in I\left(R_{E}\right)$, and $\left(x^{t}, y\right) \in P\left(R_{E}\right)$ for any $y \in B^{t} \backslash\left\{x^{t}\right\}$. Recall that in the case of the responder choice problem, $B^{t}=$ $\left\{\left(x_{\mathrm{p}}, x_{\mathrm{r}}\right),(0,0)\right\}$. To simplify notation, let $x \unrhd_{i} y$ mean that $x \geq y$ and for every $j \neq i$, $x_{i}-y_{i} \geq x_{j}-y_{j}$. With this additional nomenclature we can define quasi-monotonicity as follows:

Observation A.1. $R$ is quasi-monotone if and only if $\unrhd_{\mathrm{r}} \subseteq R$.

\section{Proof of Proposition 2.}

Definition A.1. Let $T: \mathcal{R} \rightarrow \mathcal{R}$ denote a transitive closure. Then $(x, y) \in T(R)$ if there is a sequence of elements $S=s_{1}, \ldots, s_{n}$ such that for every $j=1, \ldots, n-1$, $\left(s_{j}, s_{j+1}\right) \in R$.

Demuynck (2009) shows that there is a complete and transitive extension of a preference relation $R$ if and only if $R \preceq T(R) .{ }^{25}$ Observation A.1 implies that $R \preceq T\left(R \cup \unrhd_{\mathrm{r}}\right)$ if and only if there is a complete, transitive, and quasi-monotone extension of $R$. In the context we consider, $x$ is chosen if and only if it is strictly better than $y$, and therefore, there is a complete, transitive, and quasi-monotone relation that generates the observed choices if and only if there is a complete, transitive, and quasi-monotone extension of the revealed preference relation. This implies the following result:

\footnotetext{
${ }^{24}$ If $x \in B^{t}$, then any $y \leq x$ is also in $B^{t}$. Moreover, since we work on $\mathbf{R}^{n}$, the budget set will also include elements with negative coordinates.

${ }^{25}$ A similar result can be found in earlier papers by Szpilrajn (1930) and Richter (1966).
} 
Lemma A.1. There is a complete and weakly quasi-monotone preference relation $R$ that generates the observed choices if and only if $R_{E} \preceq T\left(R_{E} \cup \unrhd_{\mathrm{r}}\right)$.

Hence, it only remains to prove that the proposed test is equivalent to $T\left(R_{E} \cup \unrhd_{\mathrm{r}}\right)$ being an extension of $R_{E}$.

Lemma A.2. $R_{E} \preceq T\left(R_{E} \cup \unrhd_{\mathrm{r}}\right)$ if and only if

$$
\left\{x^{t}: t \in R_{x}\right\} \cap\left(A \cup\left(\bigcup_{t \in A_{x}} A^{t}\right)\right)=\emptyset
$$

Before we start the proof, note that for any $(x, y) \in T\left(R \cup \unrhd_{\mathrm{r}}\right)$, there is a shortest sequence that adds $(x, y)$ to $T\left(R \cup \unrhd_{\mathrm{r}}\right)$. By the transitivity of $\unrhd_{\mathrm{r}}$, the shortest sequence cannot contain more than one pair $s_{j}, s_{j+1}$ such that $s_{j} \unrhd_{\mathrm{r}} s_{j+1}$. Moreover, since every element is directly compared with only $x^{0}=(0,0)$, the shortest sequence cannot contain more than one pair $s_{j}, s_{j+1}$ such that $\left(s_{j}, s_{j+1}\right) \in R_{E}$. Hence, the following observation is true:

Observation A.2. If $(x, y) \in T\left(R_{E} \cup \unrhd_{\mathrm{r}}\right)$ and $x \neq y$, then the length of the shortest sequence that adds $(x, y)$ is at most three. Moreover, the sequence cannot contain more than one pair $s_{j}, s_{j+1}$ such that $\left(s_{j}, s_{j+1}\right) \in R_{E}$ or more than one pair $s_{j}, s_{j+1}$ such that $s_{j} \unrhd_{\mathrm{r}} s_{j+1}$

Given this observation, we will only refer to sequences of no more than three elements in what follows. Further, we will use the equivalent definition of being an extension, that is, $P^{-1}\left(R_{E}\right) \cap T\left(R_{E} \cup \unrhd_{\mathrm{r}}\right)=\emptyset$.

Proof of Lemma A.2. $(\Rightarrow)$ We first show that if $R_{E} \preceq T\left(R_{E} \cup \unrhd_{\mathrm{r}}\right)$, then there is no violation of the test. Assume, to the contrary, that at least one of the conditions is violated. We will show that any of these violations causes a violation of consistency with $T\left(R_{E} \cup \unrhd_{\mathrm{r}}\right)$.

Assume there exists an $x^{t}$ such that $x^{t} \in \bigcup_{t \in A_{x}} A^{t}$ and $t \in R_{x}$. The first part implies that there is a $x^{k}$ such that $\left(x^{k}, x^{0}\right) \in R_{E}$ and $x^{t} \unrhd_{\mathrm{r}} x^{k}$. Then $\left(x^{t}, x^{0}\right) \in Q M\left(R_{E}\right)$. The second part implies that $x^{t}$ was rejected: $\left(x^{0}, x^{t}\right) \in P\left(R_{E}\right)$. Therefore, $\left(x^{t}, x^{0}\right) \in$ $T\left(R_{E} \cup \unrhd_{\mathrm{r}}\right) \cap P^{-1}\left(R_{E}\right) \neq \emptyset$, i.e., $R_{E}$ is not consistent with $T\left(R_{E} \cup \unrhd_{\mathrm{r}}\right)$.

$(\Leftarrow)$ We now show that if the data pass the test, then $R_{E}$ is consistent with $T\left(R_{E} \cup \unrhd_{\mathrm{r}}\right)$ . Assume, to the contrary, that there exists some $(x, y) \in T\left(R_{E} \cup \unrhd_{\mathrm{r}}\right) \cap P^{-1}\left(R_{E}\right) \neq \emptyset$. Hence $(x, y) \in T\left(R_{E} \cup \unrhd_{\mathrm{r}}\right)$ and $(y, x) \in P\left(R_{E}\right)$. Note that by the nature of the data (binary choice between $x^{t}$ and $x^{0}$ ), either $x=x^{0}$ or $y=x^{0}$. We will show that either of 
these cases will result in failing the test.

Case 1: Assume that $x=x^{0}$, and let $y=x^{t}$. Then there is a shortest sequence $S=s_{1}, s_{2}, s_{3}$ such that $s_{1}=x^{0}$ and $s_{3}=x^{t}$. Then $\left(x^{0}, s_{2}\right) \in R_{E}$ since there is no element $x \in \mathbf{R}_{+}^{2}$ such that $\unrhd_{\mathrm{r}}$ in $\mathbf{R}_{+}^{2}$. This implies that $s_{2}=x^{s}$, i.e., a chosen point. Therefore, $\left(x^{s}, x^{t}\right) \in \unrhd_{\mathrm{r}}$, that is, $x^{s} \in A^{t}$ at the same time that $\left(x^{0}, x^{s}\right) \in R_{E}$ implies that $s \in R_{x}$. This immediately implies a contradiction.

Case 2: Assume that $x=x^{t}$, and let $y=x^{0}$. Then there is a shortest sequence $S=s_{1}, s_{2}, s_{3}$ such that $s_{1}=x^{t}$ and $s_{n}=x^{0}$. Then $\left(s_{2}, x^{0}\right) \in R_{E}$, that is, $s_{2}=x^{s}$ and it is an accepted point. ${ }^{26}$ Moreover, $x^{t} \unrhd_{\mathrm{r}} x^{s}$, that is, $x^{t} \in A^{s}$ and $x^{t}$ is a rejected point. This immediately implies a contradiction.

Proof of Proposition 3. Let us start with the proof of necessity.

Proof of Necessity. Assume, to the contrary, that the choices satisfy convex responder rationality - there is a complete, transitive, quasi-monotone, and convex preference relation $R$ that is an extension of $R_{E}$. Consider the two following cases.

Let $x^{t} \in\left\{x^{t}: t \in R_{x}\right\} \cap C H\left(A \cup\left(\bigcup_{t \in A_{x}} A_{c}^{t}\right)\right)$. This implies that $\left(x^{0}, x^{t}\right) \in$ $P\left(R_{E}\right)$, because $x^{t}$ is rejected. Considering the second part, let us assume that $x^{t} \in$ $\left(A \cup\left(\bigcup_{t \in A_{x}} A_{c}^{t}\right)\right)$, that is, there is an $x^{k}$ that is accepted: $\left(x^{k}, x^{0}\right) \in P(R)$, such that (i) $x^{t}>0$ and $x_{\mathrm{r}}^{t} \geq x_{\mathrm{p}}^{t}$, (ii) $x^{t} \geq x^{k}$ and $x_{\mathrm{r}}^{t}-x_{\mathrm{r}}^{k} \geq x_{\mathrm{p}}^{t}-x_{\mathrm{p}}^{k}$, and (iii) $x_{\mathrm{p}}^{t} \leq x_{\mathrm{p}}^{k}, x_{\mathrm{r}}^{t} x_{\mathrm{p}}^{k} \geq x_{\mathrm{p}}^{t} x_{\mathrm{r}}^{k}$. Quasi-monotonicity and (i) imply that $\left(x^{t}, x^{0}\right) \in R$. Quasi-monotonicity and (ii) imply that $\left(x^{t}, x^{k}\right) \in R$, and by transitivity, $\left(x^{t}, x^{0}\right) \in P(R)$. Convexity, quasimonotonicity, transitivity, and (iii) $\operatorname{imply}^{27}$ that $\left(x^{t}, x^{0}\right) \in R$. Another possibility is that $x^{t} \in C H\left(A \cup\left(\bigcup_{t \in A_{x}} A_{c}^{t}\right)\right) \backslash\left(A \cup\left(\bigcup_{t \in A_{x}} A_{c}^{t}\right)\right)$. Therefore, there exist $x^{k_{1}}, \ldots, x^{k_{n}}$ that are accepted and $x^{t}=\sum_{i=1}^{n} \alpha_{i} x^{k_{i}}$ such that $\alpha_{i} \geq 0$ and $\sum_{i=1}^{n} \alpha_{i}=1$; therefore, by convexity, $\left(x^{t}, x^{0}\right) \in R$.

The proof of sufficiency is analogous to the proof of Proposition 1. We use the function that induces convexity and transitivity, which also satisfies the conditions in Demuynck (2009). Therefore, the analog of Lemma A.2 is satisfied. Finally, we show that $R_{E}$ is consistent with its convex and transitive extension if and only if the convex responder rationality test is passed.

\footnotetext{
${ }^{26}$ The alternative case is $s_{2} \unrhd_{\mathrm{r}} x^{0}$, which immediately implies that $x^{t} \unrhd_{\mathrm{r}} s_{2} \unrhd_{\mathrm{r}} x^{0}$, i.e., $x^{t} \in A$ and is a rejected point.

${ }^{27}$ There exists $x=\alpha x^{0}+(1-\alpha) x^{k}$, and hence $\left(x, x^{0}\right) \in R$. Moreover, $x^{t} \geq x$ and $x_{\mathrm{r}}^{t}-x_{\mathrm{r}} \geq x_{\mathrm{p}}^{t}-x_{\mathrm{p}}$, and hence $\left(x^{t}, x\right) \in R$.
} 
Let $U_{R}(x)=\{y:(y, x) \in R\}$ denote the upper contour set of $x$. Let $L_{R}(x)=\{y$ : $(x, y) \in R\}$ denote the lower contour set of $x$. We will say that a preference relation $R$ is convex if for every $x \in X, U_{R}(x)$ is convex.

For any finite set $A \subseteq X$, let $V(A)$ denote the interior of the convex hull spanned by elements of $A$ :

$$
V(A)=\left\{x \in X: x=\sum_{y_{i} \in A} \alpha_{i} y_{i}\right\},
$$

where for all $i, \alpha_{i}>0$, and $\sum_{i} \alpha_{i}=1$.

We introduce the function $C(R)$ that Demuynck (2009) used to prove the existence of complete, transitive, and convex extensions of preferences. Consider a finite number of sequences $S^{1}, \ldots, S^{m}$. For an element $s_{j}^{i}<n_{S^{i}}$, we say that set $A$ is compatible with $s_{j}^{i}$ if

- $A \subseteq\left\{s_{v}^{k}: k \in\{1, \ldots, m\}, v \in\left\{1, \ldots, n_{S^{k}}\right\}\right\}$, and

- $s_{j+1}^{i} \in A$.

Let the sequence $S^{1}, \ldots, S^{m}$ be given. We use $\mathcal{A}\left(s_{j}^{i} ; S^{1}, \ldots, S^{m}\right)$ to denote the collection of all sets $A$ that are compatible with $s_{j}^{i}$. Set $A$ 's being compatible with an element $s_{j}^{i}$ means that there is a set of points (taken from the family of sequences) that are no worse than $s_{j}^{i}$, including $s_{j}^{i}$ and $s_{j+1}^{i}$. This allows us to represent $s_{j}^{i}$ as a convex combination of the points that are no worse than $s_{j+1}^{i}$. By convexity, this would imply that $s_{j}^{i}$ is no worse than $s_{j+1}^{i}$.

Definition A.2. Let $C(R)$ denote the convex and transitive closure of $R$. Then $(x, y) \in C(R)$ if there is a family of sequences $S^{1}, \ldots, S^{m}$ such that for all $i=1, \ldots, m$ : $s_{1}^{i}=x, s_{n^{i}}^{i}=y$, and for all $i=1, \ldots, m$ and $j=1, \ldots, n_{S^{i}}-1$ :

either $\left(s_{j}^{i}, s_{j+1}^{i}\right) \in R$, or

there is a set $A \in \mathcal{A}\left(s_{j}^{i} ; S^{1}, \ldots, S^{m}\right)$ such that $s_{j}^{i} \in V(A)$.

The following result can be immediately deduced from the results in Demuynck (2009) and Observation A.1:

Lemma A.3. There exists a complete, convex, and weakly quasi-monotone preference relation that generates the observed choices if and only if $R_{E} \preceq C\left(R_{E} \cup \unrhd_{\mathrm{r}}\right)$.

Therefore, we need to prove that consistency with $C$ is equivalent to the test of convex responder rationality. Demuynck (2009) shows that $C(R)=R$ if and only if $R$ is transitive and convex. Therefore, the following corollary follows from Lemma A.3:

Corollary A.1. If $R$ is convex and transitive, then it is consistent with $C(R)$. 
We now construct a convex, transitive, and quasi-monotone extension of $R_{E}$ and show that if the test of convex responder rationality is satisfied, then $R_{E}$ is consistent with it. This allows us to complete the proof by applying Corollary A.1. We construct a convex, transitive, and quasi-monotone extension of $R_{E}$ in two steps. First, we construct $Q_{E}$, which is quasi-monotone and convex, but not yet transitive. Second, we construct $T C\left(Q_{E}\right)$, which is convex, transitive, and quasi-monotone.

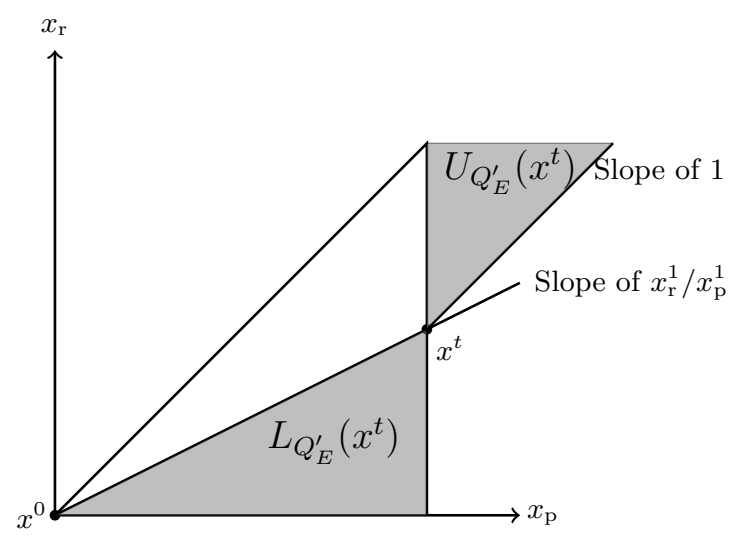

(a) $x^{t}$ is accepted

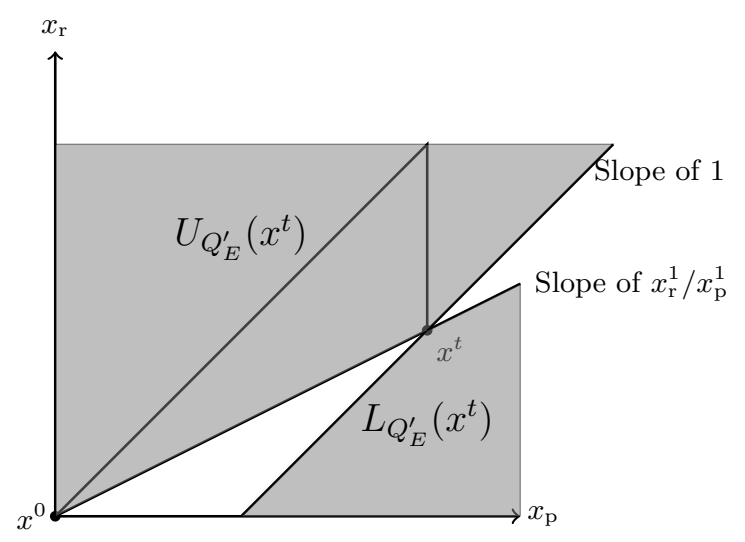

(b) $x^{t}$ is rejected

Figure A.1. Constructing $Q_{E}^{\prime}$

To construct the relation $Q_{E}$, which is a convex and quasi-monotone extension of $R_{E}$, we need to construct the intermediate relation $Q_{E}^{\prime}$, which is a substantial subrelation of $Q_{E}$. If allocation $x^{t} \in C H\left(A \cup\left(\bigcup_{t \in A_{x}} A_{c}^{t}\right)\right)$, then $L_{Q_{E}^{\prime}}\left(x^{t}\right)=\left\{x: x_{\mathrm{p}} \leq x_{\mathrm{p}}^{t}, x_{\mathrm{r}} x_{\mathrm{p}}^{t} \leq x_{\mathrm{p}} x_{\mathrm{r}}^{t}\right\}$ and $U_{Q_{E}^{\prime}}\left(x^{t}\right)=\left\{x: x \unrhd_{\mathrm{r}} x^{t}\right\}$. If allocation $x^{t} \in \bigcup_{t \in R_{x}} R_{c}^{t}$, then $L_{Q_{E}^{\prime}}\left(x^{t}\right)=\left\{x: x^{t} \unrhd_{\mathrm{r}} x\right\} \cup\{x:$ $\left.x_{\mathrm{p}} \geq x_{\mathrm{p}}^{t}, x_{\mathrm{r}} x_{\mathrm{p}}^{t} \geq x_{\mathrm{p}} x_{\mathrm{r}}^{t}\right\}$ and $U_{Q_{E}^{\prime}}\left(x^{t}\right)=\left\{x: x_{\mathrm{p}} \leq x_{\mathrm{p}}^{t}, x_{\mathrm{r}} x_{\mathrm{p}}^{t} \leq x_{\mathrm{p}} x_{\mathrm{r}}^{t}\right\} \cup\left\{x: x \unrhd_{\mathrm{r}} x^{t}\right\}$. In addition, $L_{Q_{E}^{\prime}}\left(x^{0}\right)=\left(\bigcup_{t \in R_{x}} R_{c}^{t}\right)$ and $U_{Q_{E}^{\prime}}\left(x^{0}\right)=C H\left(A \cup\left(\bigcup_{t \in A_{x}} A_{c}^{t}\right)\right)$. Figure A.1 illustrates the construction of $Q_{E}^{\prime}$; the upper and lower contour sets are the shadowed areas. $Q_{E}^{\prime}$ is an intermediate relation that is the most important addition to $R_{E}$ (the revealed preference relation). We then construct $Q_{E}$ as the union of $Q_{E}^{\prime}, R_{E}$, and the quasi-monotone relation, i.e., $Q_{E}=Q_{E}^{\prime} \cup \unrhd_{\mathrm{r}} \cup R_{E}$.

Note that $\unrhd_{\mathrm{r}}$ is also a binary relation, so that this union is well-defined. This guarantees that $Q_{E}$, as well as every preference relation that extends $Q_{E}$, is quasi-monotone. Moreover, it can easily be seen that $Q_{E}$ is convex, since all the upper contour sets are convex by construction.

To define the convex and transitive extension, we use the following inductive procedure. Let $C_{0}=T(R)$, and let $C_{i}=T\left(C_{i-1}(R) \cup\left[\bigcup_{x \in \Theta\left(C_{i-1}(R)\right)}\left\{(x, y): x \in C H\left(U_{C_{i-1}}(y)\right)\right\}\right]\right)$, where $\Theta(R)=\left\{x: L_{R}(x) \neq \emptyset\right.$ and $\left.L_{R}(x) \neq\{x\}\right\}$. Then $T C(R)=T\left(\bigcup_{i \in \mathbf{N}} C_{i}(R)\right)$. This procedure simply takes, at every step, the transitive closure of the convexification of the previous relation $C_{i-1}(R)$. Convexification is simply taking the convex hull of 
every upper contour set. We next show that $T C(R)$ is convex and transitive, and hence consistent with $C$.

Lemma A.4. $T C(R)$ is convex and transitive.

Proof. By construction, $T C(R)$ is transitive, since it is defined as a transitive closure of the union of preference relations. Hence, it remains to show that $T C(R)$ is convex. Assume, to the contrary, that there is an $x \in X$ such that there are $y_{1}, \ldots, y_{n} \in$ $U_{T C(R)}(x)$ and there is a $y=\sum_{i} \alpha_{i} y_{i}, a_{i} \geq 0, \sum_{i} \alpha_{i}=1$ such that $y \notin U_{T C(R)}(x)$.

First, we show that $T C(R) \backslash\left(\bigcup_{i \in \mathbf{N}} C_{i}(R)\right)=\emptyset$. Assume, to the contrary, that there is a $\left(y_{i}, x\right) \in T C(R) \backslash\left(\bigcup_{i \in \mathbf{N}} C_{i}(R)\right)$. By the definition of transitive closure, this means that there is a sequence with $S=s_{1}, \ldots, s_{n}, s_{1}=y_{i}$ and $s_{n}=x$ such that for every $j=1, \ldots, n-1,\left(s_{j}, s_{j+1}\right) \in \bigcup_{i \in \mathbf{N}} C_{i}(R)$. By the construction of $\bigcup_{i \in \mathbf{N}} C_{i}(R)$, for every $j=1, \ldots, n-1$, there is a $k_{j}$ such that $\left(s_{j}, s_{j+1}\right) \in C_{k_{j}}(R)$. Let $m$ be the maximum integer such that $m \geq k_{j}$ for every $j{ }^{28}$ Therefore, $\left(y_{i}, x\right) \in C_{m+1}(R)$.

Second, we show that if $y_{1}, \ldots, y_{n} \in U_{T C(R)}(x)$, then $y \in U_{T C(R)}(x)$ for all $y=\sum_{i} \alpha_{i} y_{i}$ such that $\alpha_{i}>0$ and $\sum_{i} \alpha_{i}=1$. Assume, to the contrary, that there is $y=\sum_{i} \alpha_{i} y_{i}$ such that $\alpha_{i}>0, \sum_{i} \alpha_{i}=1$, and $y \notin U_{T C(R)}(x)$. Note that by the construction of $T C(R)$, for every $i=1, \ldots, n-1$, there is $k_{i}$ such that $y_{i} \in U_{C_{k_{i}}}(R)(x)$. Let $m$ be the maximum integer such that $m \geq k_{i}$ for every $i$. Therefore, $y \in U_{C_{m+1}(R)}(x)$, since the upper contour set of $C_{m+1}(R)$ is obtained by taking a convex hull of all upper contour sets of $C_{m}(R)$.

Therefore, $T C\left(Q_{E}\right)$ is a convex, transitive, and quasi-monotone relation (see Lemma A.4 and Observation A.1). Hence, it remains to show that $R_{E} \preceq T C\left(Q_{E}\right)$. Let us start with a supplementary result that shows that $R_{E} \preceq Q_{E}$.

Lemma A.5. If

$$
\left\{x^{t}: t \in R_{x}\right\} \cap C H\left(A \cup\left(\bigcup_{t \in A_{x}} A_{c}^{t}\right)\right)=\emptyset
$$

then $R_{E} \preceq Q_{E}$.

Proof. Assume, to the contrary, that there are $(y, x) \in P\left(R_{E}\right)$ and $(x, y) \in Q_{E}$ and consider the following cases.

Case 1: $y=x^{t}$ and $x=x^{0}$. If $\left(x^{0}, x^{t}\right) \in Q_{E}$, then $x^{0} \in U_{Q_{E}}\left(x^{t}\right)$. But by the construction of $Q_{E}$ there are only three possibilities for $x^{0} \in U_{Q_{E}}\left(x^{t}\right)$ : (i) $\left(x^{0}, x^{t}\right) \in R_{E}-$ this

\footnotetext{
${ }^{28} m$ exists because all the sequences are finite.
} 
is impossible because $\left(x^{t}, x^{0}\right) \in P\left(R_{E}\right)$; (ii) $\left(x^{0}, x^{t}\right) \in Q_{E}^{\prime}$ - this is impossible because it implies that $x^{t}$ is rejected; and (iii) $\left(x^{0}, x^{t}\right) \in \unrhd_{\mathrm{r}}-$ this is impossible, because we have $x^{t}>x^{0}$.

Case 2: $y=x^{0}$ and $x=x^{t}$. If $\left(x^{t}, x^{0}\right) \in Q_{E}$, then $x^{t} \in U_{Q_{E}}\left(x^{0}\right)$. But by the construction of $Q_{E}$ there are only three possibilities: (i) $\left(x^{t}, x^{0}\right) \in R_{E}$ - this is impossible because $\left(x^{0}, x^{t}\right) \in P\left(R_{E}\right)$; (ii) $\left(x^{t}, x^{0}\right) \in Q_{E}^{\prime}$ - this is impossible because $x^{t}$ is a rejected point; and (iii) $\left(x^{t}, x^{0}\right) \in \unrhd_{\mathrm{r}}-$ this is impossible because it implies that $x^{t} \in A$.

Lemma A.6. If

$$
\left\{x^{t}: t \in R_{x}\right\} \cap C H\left(A \cup\left(\bigcup_{t \in A_{x}} A_{c}^{t}\right)\right)=\emptyset
$$

then $R_{E} \preceq T C\left(Q_{E}\right)$.

Before proceeding with the proof, we make two observations.

Observation A.3. If

$$
\left\{x^{t}: t \in R_{x}\right\} \cap C H\left(A \cup\left(\bigcup_{t \in A_{x}} A_{c}^{t}\right)\right)=\emptyset
$$

then for every $t \in A_{x}, U_{Q_{E}}\left(x^{t}\right)=U_{T C\left(Q_{E}\right)}\left(x^{t}\right)$.

Observation A.3 says that if the data are consistent with the test, then the upper contour sets of the accepted points remain unaffected by taking the convex closure. This follows from the construction of $Q_{E}$. Moreover, the only points that can be better than $x^{t}$ are other accepted points. Hence, if the data are consistent with the test, then the only points that can be preferred to the accepted point are $y \unrhd_{\mathrm{r}} x^{t}$.

Observation A.4. If

$$
\left\{x^{t}: t \in R_{x}\right\} \cap C H\left(A \cup\left(\bigcup_{t \in A_{x}} A_{c}^{t}\right)\right)=\emptyset
$$

then $U_{Q_{E}}\left(x^{0}\right)=U_{C\left(Q_{E}\right)}\left(x^{0}\right)$.

Observation A.4 says that if the data are consistent with the test, then the upper contour set of $x^{0}$ remains unaffected by taking the convex closure. This also follows from the construction of $Q_{E}$. If the data are consistent with the test, then the only points that are better than $x^{0}$ are the accepted points, points that are better than the accepted points, and $y \unrhd_{\mathrm{r}} x^{0}$; however, $U_{Q_{E}}\left(x^{0}\right)$ already includes the convex hull of these points. 
Therefore, since the upper contour sets of accepted points remain unchanged, the upper contour set of zero remains unchanged as well. ${ }^{29}$

Proof of Lemma A.6. Assume, to the contrary, that $(y, x) \in P\left(R_{E}\right)$ and $(x, y) \in T C\left(Q_{E}\right)$. Note that $(x, y) \notin Q_{E}$, since $R_{E} \preceq Q_{E}$.

Case 1: $y=x^{t}$ and $x=x^{0}$. Note that $\left(x^{0}, x^{t}\right) \in T C\left(Q_{E}\right)$ implies that $x^{0} \in$ $U_{T C\left(Q_{E}\right)}\left(x^{t}\right)$. However, $x^{t}$ is an accepted point, and therefore Observation A.3 implies that $U_{T C\left(Q_{E}\right)}\left(x^{t}\right)=U_{Q_{E}}\left(x^{t}\right)$. Hence $\left(x^{0}, x^{t}\right) \in Q_{E}$, which is a contradiction.

Case 2: $y=x^{0}$ and $x=x^{t}$. Note that $\left(x^{t}, x^{0}\right) \in T C\left(Q_{E}\right)$ implies that $x^{t} \in$ $U_{T C\left(Q_{E}\right)}\left(x^{0}\right)$. However, $x^{t}$ is an accepted point, and therefore, Observation A.4 implies that $U_{T C\left(Q_{E}\right)}\left(x^{0}\right)=U_{Q_{E}}\left(x^{0}\right)$. Hence $\left(x^{t}, x^{0}\right) \in Q_{E}$, which is a contradiction.

We can complete the proof by applying Lemma A.6 and Lemma A.3.

Proof of Corollary 1. Without loss of generality, assume that all the beliefs can be expressed as integers between 0 and 100. While we assume a finite population size to avoid complicating the notation, all of the results hold for a continuous population. We then construct an incomplete preference relation that is consistent with $T\left(R \cup \unrhd_{\mathrm{r}}\right)$ and apply Lemma A.1, which guarantees the existence of a complete transitive and quasimonotone extension of $R$. The construction is done in a way that guarantees that the frequency of an outcome being accepted corresponds to the elicited beliefs. Let $\mathcal{P}\left(x^{t}\right)$, with typical element $a$, denote the set of agents who prefer $x^{t}$ to zero. We assume that if $x^{t}$ is an element on the $45^{\circ}$ line and $a \notin \mathcal{P}\left(x^{t}\right)$, then $a$ is indifferent between zero and $x^{t}$. This guarantees that she will reject $x^{t}$ but keeps her preference relation consistent with $\unrhd_{\mathrm{r}}$. Since the set of beliefs is well-ordered, we can reorder its elements such that $1-b^{t}<1-b^{s}$ if $t<s$. Let $\mathcal{P}\left(\mathbf{x}^{1}\right)$ be such that $\left|\mathcal{P}\left(\mathbf{x}^{1}\right)\right|=1-b^{1}$, which can be done by taking a random set of $1-b^{1}$ subjects from the population. $\mathcal{P}\left(\mathbf{x}^{t}\right)$ can be constructed using the following recursive rule: (1) $\left|\mathcal{P}\left(\mathbf{x}^{t}\right)\right|=1-b^{t} ;(2) \mathcal{P}\left(\mathbf{x}^{s}\right) \subseteq \mathcal{P}\left(\mathbf{x}^{t}\right)$ for all $s<t$; (3) if $1-b^{t}=1-b^{s}$, then $\mathcal{P}\left(\mathbf{x}^{s}\right)=\mathcal{P}\left(\mathbf{x}^{t}\right)$.

Lemma A.7. For every player a in the population, the constructed preference relation is consistent with $T\left(R \cap \unrhd_{\mathrm{r}}\right)$.

Proof. We prove this Lemma using Lemma A.2. Assume, to the contrary, that there are $\mathbf{x}^{t}$ and $\mathbf{x}^{s}$ such that $\mathbf{x}^{s} \unrhd_{\mathrm{r}} \mathbf{x}^{t}, a \notin \mathcal{P}\left(\mathbf{x}^{s}\right)$, and $a \in \mathcal{P}\left(x^{t}\right)$. The last two conditions imply that $\mathbf{x}^{t}$ is preferred to zero, and zero is preferred to $\mathbf{x}^{s}$ by the construction of

\footnotetext{
${ }^{29} \mathrm{An}$ additional observation is implicit in this explanation, namely, the upper contour sets of $y \unrhd_{\mathrm{r}} x^{0}$ also remain unchanged.
} 
the preference relation. However, the conditions also imply that $\mathcal{P}\left(\mathbf{x}^{t}\right) \subseteq \mathcal{P}\left(\mathbf{x}^{s}\right)$, and therefore, $a \in \mathcal{P}\left(\mathbf{x}^{s}\right)$, which is a contradiction.

Hence, the proof can be completed using Lemma A.1.

Proof of Corollary 2. We first establish the necessity of the conditions. Assume that there is a population of convex responder rational agents such that the frequency of choices coincides with the elicited beliefs. Let $A_{c}(S)$ denote $C H\left(A \cup\left(\bigcup_{x^{t} \in S} A_{c}^{t}\right)\right)$, and let $\partial A_{c}(S)$ denote the southwest border of the convex hull. Then $\bigcap_{x^{s} \in S \cap \partial A_{c}(S)} \mathcal{P}\left(x^{s}\right)$ is the set of people who prefer $x^{s}$ to zero for every point that lies within the boundary of the convex hull. Then the convexity of preferences implies that $\mathcal{P}\left(x^{t}\right) \subset \bigcap_{x^{s} \in S \cap \partial A_{c}(S)} \mathcal{P}\left(x^{s}\right)$ for every $x^{t} \in A_{c}(S) \backslash \partial A_{c}(S)$. Therefore, $b^{t} \leq \max _{x^{s} \in S \cap \partial A_{c}(S)}\left\{b^{s}\right\}$ for every $x^{t} \in A_{c}(S)$. In particular, this implies that $\max _{x^{s} \in S \cap \partial A_{c}(S)}\left\{b^{s}\right\}=\max _{x^{s} \in S}\left\{b^{s}\right\}$. Therefore, we can conclude that $b^{t} \leq \max _{x^{s} \in S}\left\{b^{s}\right\}$ for every $x^{t} \in A_{c}(S)$.

We now establish the sufficiency of the conditions. Since the set of beliefs is wellordered, we can reorder its elements such that $1-b^{t}<1-b^{s}$ if $t<s$. Let $\mathcal{P}\left(x^{1}\right)$ be such that $\left|\mathcal{P}\left(x^{1}\right)\right|=1-b^{1}$, which can be done by taking a random set of $1-b^{1}$ subjects from the population. $\mathcal{P}\left(x^{t}\right)$ can be constructed using the following recursive rule: (1) $\left|\mathcal{P}\left(x^{t}\right)\right|=1-b^{t}$; (2) $\mathcal{P}\left(x^{s}\right) \subseteq \mathcal{P}\left(x^{t}\right)$ for all $s<t$; (3) if $1-b^{t}=1-b^{s}$, then $\mathcal{P}\left(x^{s}\right)=\mathcal{P}\left(x^{t}\right)$.

Lemma A.8. For every player a in the population, the constructed preference relation is consistent with convex responder rationality.

Proof. Assume, to the contrary, that there is a violation, i.e., $x^{s} \in S \in 2^{C \backslash x^{t}}$ and $x^{t} \in A_{c}(S)$. That is, $a \in \mathcal{P}\left(x^{s}\right)$ for every $x^{s} \in S$ and $a \notin \mathcal{P}\left(x^{t}\right)$. These conditions imply that $b^{t} \leq \max _{x^{s} \in S}\left\{b^{s}\right\}=b^{r}$. Therefore, $\mathcal{P}\left(x^{r}\right) \subseteq \mathcal{P}\left(x^{t}\right)$ by the construction. This is a contradiction.

Hence, we can complete the proof by applying Proposition 3.

\section{Appendix B: Additional Data}

We conducted two additional experimental sessions at Texas A\&M University with a total of 40 subjects. In addition to the ultimatum games, we conducted incentivized point belief elicitation using the binarized scoring procedure from Hossain and Okui (2013).

Table B.1 reproduces Table 1. Recall that a proposer is called generous if she offers more than the minimum possible allocation. A responder is called a rejector if she rejects the minimum allocation. Table B.1 shows that subjects at Texas A\&M offer more 


\begin{tabular}{|c|c|c|c|c|c|c|c|c|c|}
\hline \multirow{3}{*}{$\begin{array}{l}m=x_{\mathrm{p}}+p x_{\mathrm{r}} \\
\text { with }(m, p)= \\
\end{array}$} & \multicolumn{4}{|c|}{ Proposed $x_{\mathrm{r}}$} & \multicolumn{4}{|c|}{ Highest rejected $x_{\mathrm{r}}$} & \multirow{3}{*}{$\begin{array}{l}\text { No cut- } \\
\frac{\text { off rule }}{\underline{n}}\end{array}$} \\
\hline & \multicolumn{2}{|c|}{ All proposers } & \multicolumn{2}{|c|}{ Generous } & \multicolumn{2}{|c|}{ All responders } & \multicolumn{2}{|c|}{ Rejectors } & \\
\hline & Mean & $\underline{\text { St.Dev }}$ & $\underline{\% \text {-age }}$ & Mean & Mean & $\underline{\text { St.Dev }}$ & $\underline{\% \text {-age }}$ & Mean & \\
\hline$\left(\$ 12, \frac{1}{5}\right)$ & $\$ 16.44$ & $\$ 16.21$ & $97.5 \%$ & $\$ 16.80$ & $\$ 3.90$ & $\$ 1.78$ & $100.0 \%$ & $\$ 3.90$ & 4 \\
\hline$\left(\$ 24, \frac{1}{3}\right)$ & $\$ 20.85$ & $\$ 12.65$ & $100.0 \%$ & $\$ 20.85$ & $\$ 7.30$ & $\$ 2.98$ & $100.9 \%$ & $\$ 7.30$ & 5 \\
\hline$\left(\$ 24, \frac{1}{2}\right)$ & $\$ 16.35$ & $\$ 8.36$ & $97.5 \%$ & $\$ 16.72$ & $\$ 6.72$ & $\$ 3.16$ & $100.0 \%$ & $\$ 6.72$ & 6 \\
\hline$(\$ 36,1)$ & $\$ 15.83$ & $\$ 4.95$ & $97.5 \%$ & $\$ 16.20$ & $\$ 8.24$ & $\$ 4.04$ & $100.0 \%$ & $\$ 8.24$ & 6 \\
\hline$(\$ 48,1)$ & $\$ 20.40$ & $\$ 7.99$ & $97.5 \%$ & $\$ 20.90$ & $\$ 8.81$ & $\$ 4.00$ & $100.0 \%$ & $\$ 8.81$ & 6 \\
\hline$(\$ 60,1)$ & $\$ 24.68$ & $\$ 8.12$ & $95.0 \%$ & $\$ 25.92$ & $\$ 10.31$ & $\$ 6.37$ & $100.0 \%$ & $\$ 10.31$ & 6 \\
\hline$(\$ 48,2)$ & $\$ 12.55$ & $\$ 5.03$ & $90.0 \%$ & $\$ 13.83$ & $\$ 7.79$ & $\$ 3.61$ & $100.0 \%$ & $\$ 7.79$ & 5 \\
\hline$(\$ 72,3)$ & $\$ 13.85$ & $\$ 5.92$ & $90.0 \%$ & $\$ 15.28$ & $\$ 9.00$ & $\$ 4.20$ & $100.0 \%$ & $\$ 9.00$ & 5 \\
\hline$(\$ 60,5)$ & $\$ 7.84$ & $\$ 3.08$ & $92.5 \%$ & $\$ 8.43$ & $\$ 6.44$ & $\$ 2.02$ & $100.0 \%$ & $\$ 6.44$ & 7 \\
\hline All 9 budgets & $\$ 16.53$ & $\$ 10.00$ & $95.28 \%$ & $\$ 17.30$ & $\$ 7.65$ & $\$ 4.28$ & $100.0 \%$ & $\$ 7.65$ & 7 \\
\hline
\end{tabular}

TABLE B.1. Summary of Proposer and Responder Behavior

money as proposers and reject higher offers as responders. Hence, the two populations have different preferences. In what follows, we analyze the consistency of proposers' and responders' choices and beliefs.

\begin{tabular}{cc|cc}
\hline \hline CCEI & Number of Subjects & Bronars' power & e.d.f. power \\
\hline 1 & 34 & .9012 & .7870 \\
{$[1-\varepsilon, 1)$} & 4 & .8352 & .4893 \\
{$[.9,1-\varepsilon)$} & 1 & .5882 & .3368 \\
{$[.8, .9)$} & 0 & .3018 & .1196 \\
{$[0, .8)$} & 1 & & \\
\hline \hline
\end{tabular}

TABLE B.2. Proposer Rationality

Table B.2 shows the pass rates for consistency with proposer rationality. The pass rate is $85 \%$ and increases to $95 \%$ if we allow for small violations. The pass rates are not significantly different from those obtained in the original data. ${ }^{30}$

Table B.3 presents the results on responder rationality and convex responder rationality for the new data. The results are qualitatively similar to the original sample: subjects' beliefs are consistent with responder rationality but not with convex responder rationality. Forty-five percent of the subjects's beliefs are consistent with responder rationality, and this number goes up to $78 \%$ if we allow for no more than five mistakes.

\footnotetext{
${ }^{30}$ Further, we compare pass rates using a t-test, the Wilcoxon rank sum test, and estimated confidence intervals based on the Clopper-Pearson procedure.
} 


\begin{tabular}{ccccc}
\hline \multirow{2}{*}{ Number of Violations } & \multicolumn{2}{c}{ Responder Rationality } & \multicolumn{2}{c}{ Convex Responder Rationality } \\
\cline { 2 - 5 } & Number of Subjects & Percent of Subjects & Number of Subjects & Percent of Subject \\
\hline 0 & 18 & $45 \%$ & 8 & $20 \%$ \\
$\leq 5$ & 31 & $78 \%$ & 8 & $20 \%$ \\
$\geq 6$ & 9 & $22 \%$ & 32 & $80 \%$ \\
\hline
\end{tabular}

Power of Test

\begin{tabular}{ccccc} 
& \multicolumn{2}{c}{ Proposer Rationality } & \multicolumn{2}{c}{ Convex Proposer Rationality } \\
\cline { 2 - 4 } Test & Power of Test & $\begin{array}{c}\text { Average Number } \\
\text { of Violations (std) }\end{array}$ & $\begin{array}{c}\text { Power of Test } \\
\text { Average Number } \\
\text { of Violations (std) }\end{array}$ \\
\hline Random & 1.0000 & $47.0850(4.8914)$ & 1.0000 & $58.7460(5.6078)$ \\
Random Cutoff & 1.0000 & $34.1910(9.8979)$ & 1.0000 & $62.8880(10.8994)$ \\
e.d.f. & 0.9990 & $15.4810(3.3961)$ & 1.0000 & $32.4580(3.7548)$ \\
e.d.f. Cutoff & 0.9180 & $11.4410(4.5483)$ & 1.0000 & $38.0280(6.1267)$ \\
\hline
\end{tabular}

TABLE B.3. Responder Rationality

At the same time, $80 \%$ make six or more mistakes according to the convex responder rationality. The original sample contains a larger share of subjects whose beliefs are consistent with responder rationality, and a larger share of its population have no more than five violations of responder rationality. However, neither of the differences is statistically significant. There is no significant difference for consistency with convex responder rationality if we compare exact pass rates, while the difference is significant if we relax the notion of rationality and allow for no more than five violations.

\begin{tabular}{ccc}
\hline \hline & $\begin{array}{c}\text { Consistent with } \\
\text { Responder Rationality }\end{array}$ & $\begin{array}{c}\text { Inconsistent with } \\
\text { Responder Rationality }\end{array}$ \\
\cline { 2 - 3 } Consistent with Proposer Rationality $(C C E I=1)$ & $17(42 \%)$ & $21(52 \%)$ \\
Inconsistent with Proposer Rationality $(C C E I \neq 1)$ & $1(3 \%)$ & $1(3 \%)$ \\
\hline \hline
\end{tabular}

TABLE B.4. Cross Table: Proposer Rationality and Responder Rationality

\begin{tabular}{ccc}
\hline \hline & $\begin{array}{c}\text { Consistent with } \\
\text { Responder Rationality }\end{array}$ & $\begin{array}{c}\text { Inconsistent with } \\
\text { Responder Rationality }\end{array}$ \\
\cline { 2 - 3 } Consistent with Proposer Rationality $(C C E I \geq 1-\varepsilon)$ & $29(73 \%)$ & $9(22 \%)$ \\
Inconsistent with Proposer Rationality $(C C E I<1-\varepsilon)$ & $2(5 \%)$ & $0(0 \%)$ \\
\hline \hline
\end{tabular}

TABLE B.5. Cross Table: Weak Proposer Rationality $(C C E I \geq 1-\varepsilon)$ and Responder Rationality (number of violations $\leq 5$ )

Tables B.4 and B.5 present results on the interdependence between proposer and responder rationality. The results are similar to those obtained in the original data.

Figure B.1 shows the mean and the 95\% confidence intervals for the elicited beliefs. Recall that we elicited beliefs regarding the probability that an offer is rejected. 


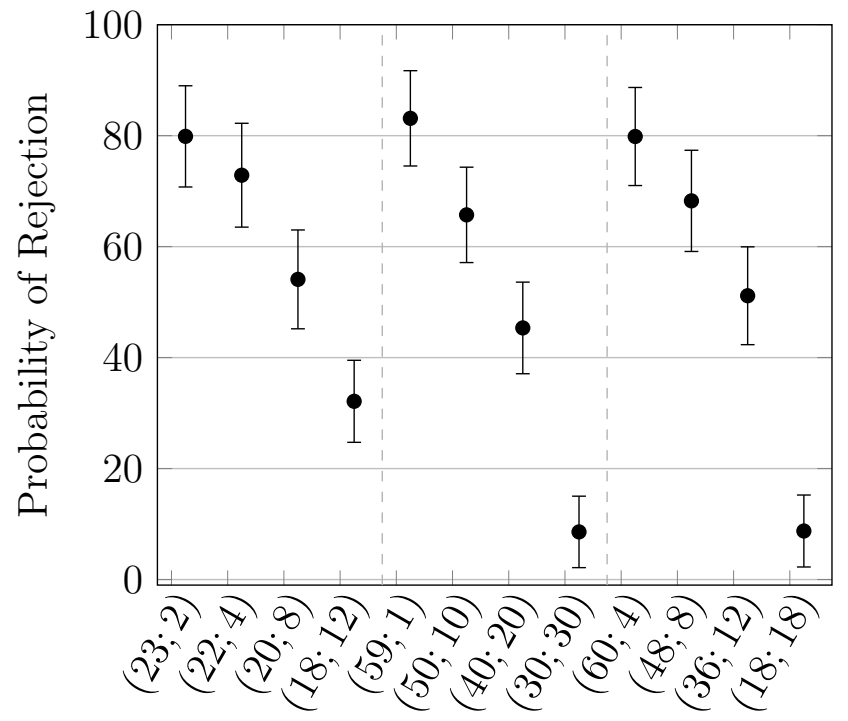

Figure B.1. Average Beliefs and Confidence Intervals

Therefore, higher numbers imply a higher probability of rejection. The wide confidence intervals imply a high degree of heterogeneity of beliefs. At the same time, the beliefs are monotone within a menu (blocks of four) but not between the menus. In particular, beliefs not only depend on the responder's payoff, but also on the proposer's payoff- the probability of rejecting is higher for $(36,12)$ than for $(18,12)$. However, this is not always the case - the probabilities of accepting equal split outcomes $(18,18)$ and $(30,30)$ are the same, regardless of the different price regimes ( $p=1$ in the first case and $p=3$ in the second case). Finally, we found that the average beliefs are consistent with both responder rationality and convexity. ${ }^{31}$

We now turn to the analysis of beliefs at the individual level. To make this comparable to the original experiment, which elicited beliefs in intervals, we introduce a slack variable $e$ or measurement error term for beliefs. Without a measurement error, the probability of rejecting offer $x(q(x))$ is higher than the probability of rejecting offer $x^{\prime}$ $\left(q\left(x^{\prime}\right)\right)$ if and only if $q(x)>q\left(x^{\prime}\right)$. Given the slack variable, the probability of rejecting offer $x(q(x))$ is higher than the probability of rejecting offer $x^{\prime}\left(q\left(x^{\prime}\right)\right)$ if and only if $q(x)>q\left(x^{\prime}\right)+e$ (we denote this as $\left.q(x)>_{e} q\left(x^{\prime}\right)\right)$. We consider three levels of slack:

\footnotetext{
${ }^{31} \mathrm{An}$ average belief $b_{i}$ is greater than belief $b_{j}$ if it is greater at the $5 \%$ significance level. For instance, the probability of rejecting $(23,2)$ is significantly greater than the probability of rejecting $(20,8)$, while it is not significantly greater than the probability of rejecting $(22,4)$. If we do not account for confidence intervals, then average beliefs are not consistent with responder rationality. In addition, we conduct power estimates using Bronars' and the e.d.f. tests, using repeated sampling of 40 observations out of the populations of 10000 simulated observations. All power tests return 1.0000-there is zero chance that e.d.f. or uniform random subjects would generate beliefs that would be on average consistent.
} 
no slack, five percentage points, and thirty percentage points. A slack of 5 percentage points reflects the observed variation in the experiment. A slack of 30 percentage points reflects the span of intervals in the original experiment.

\begin{tabular}{|c|c|c|}
\hline Slack & $\begin{array}{l}\text { Beliefs Consistent with } \\
\text { Responder Rationality }\end{array}$ & $\begin{array}{c}\text { Beliefs Consistent with } \\
\text { Convex Responder Rationality }\end{array}$ \\
\hline$e=0$ & $22(40 \%)$ & $1(2.5 \%)$ \\
\hline$e \leq 5$ & $30(75 \%)$ & $1(2.5 \%)$ \\
\hline$e \leq 30$ & $37(93 \%)$ & $3(8.5 \%)$ \\
\hline \multicolumn{3}{|l|}{ Power Analysis } \\
\hline Test & Responder Ratonality & Convex Responder Rationality \\
\hline Bronars \& $e=0$ & 0.9900 & 1.0000 \\
\hline Bronars \& $e \leq 5$ & 0.9830 & 1.0000 \\
\hline Bronars \& $e \leq 30$ & 0.8610 & 0.8610 \\
\hline e.d.f. $\& e=0$ & 0.8822 & 1.0000 \\
\hline e.d.f. \& $e \leq 5$ & 0.8214 & 1.0000 \\
\hline e.d.f. $\& e \leq 30$ & 0.4982 & 1.0000 \\
\hline
\end{tabular}

TABLE B.6. Belief Consistency

Table B.6 presents the results on belief consistency. Only $40 \%$ of the subjects have consistent beliefs unless we allow for measurement error. This proportion is statistically different from the proportion of subjects with consistent beliefs in the original experiment (81\%). At the same time, $75 \%$ of the subjects have consistent beliefs given the slack of 5 percentage points. This difference is not statistically different from the proportion of subjects with consistent beliefs in the original data. The hypothesis that the beliefs are consistent with convex responder rationality is rejected decisively. Furthermore, with a slack of 30 percentage points, only 3 out of 40 subjects have beliefs that are consistent with convex responder rationality. The difference in pass rates for convex responder rationality is not statistically significantly different from the original data for any of the levels of slack presented. Power analysis conducted using Bronars' and e.d.f. power tests shows that the tests can successfully distinguish between real consistent behavior and the randomly consistent behavior.

Finally, we investigate which assumptions of proposer rationality are more likely to fail by looking at the patterns of behavior in the three experimental tasks. We find that $23(58 \%)$ of the subjects passed all three tests, 6 (15\%) failed only consistency of beliefs, and $2(5 \%)$ failed only proposer rationality (violate stochastic dominance preferences). In addition, 9 subjects' beliefs are consistent with proposer rationality but 
are not consistent with responder rationality. Five of these 9 subjects passed the belief consistency test. These subjects seem to have consistent beliefs and behave rationally, but according to a weaker notion of monotonicity. 


\section{References}

Afriat, S. 1967. "The Construction of a Utility Function From Expenditure Data," International Economic Review, 8, 67-77.

Afriat, S. 1972. "Efficiency estimation of production functions," International Economic Review, 14(2) 568-98.

Afriat, S. 1973. "On a System of Inequalities in Demand Analysis: An Extension of the Classical Method," International Economic Review, 13(3) 460-72.

Andreoni, J., M. Castillo and R. Petrie. 2003. "What do Bargainers' Preferences Look Like? Exploring a Convex Ultimatum Game" American Economic Review, v. 93(3), $672-685$.

Andreoni, J., Miller, J., 2002. Giving According to GARP: An Experimental Test of the Consistency of Preference for Altruism. Econometrica, 70(3), 737-753.

Andreoni, J. and W. Harbaugh. 2009. "Unexpected Utility: Experimental Tests of Five Key Questions about Preferences over Risk." UCSD mimeo.

Battalio, R., J. Kagel, R. Winkler, E. Fisher, R. Basmann and L. Krasner. 1973. “A Test of Consumer Demand Theory Using Observations of Individual Consumer Purchases." Western Economic Journal, 11(4), 411-28.

Benjamin, D. 2015. "Distributional Preferences, Reciprocity-Like Behavior, and Efficiency in Bilateral Exchange," American Economic Journal: Microeconomics, 7(1), 70-98.

Berg, J., J. Dickhaut, and K.McCabe. 1995. "Trust, Reciprocity, and Social History," Games and Economic Behavior, Vol. 10, No. 1, pp. 122-42.

Bolton, G. and A. Ockenfels. 2000. "ERC: A Theory of Equity, Reciprocity, and Competition," American Economic Review, Vol. 90, No. 1, pp. 166-193.

Blow, L., M. Browning, and I. Crawford. 2008. "Revealed Preference Analysis of Characteristics Models." Review of Economic Studies, 75(2), 371-89.

Bronars, S. 1987. "The Power of Nonparametric Tests of Preference Maximization", Econometrica, 55, 693-698.

Brown, D. and R. Matzkin. 1996. "Testable Restrictions on the Equilibrium Manifold," Econometrica, 64, 1249-62. 
Camerer, C., 2003. Social Preferences in Dictator, Ultimatum, and Trust Games. In: Behavioral Game Theory, Princeton University Press, Princeton, NJ.

Carvajal, A., Deb, R., Fenske, J., and Quah, J. K. H. 2013. "Revealed preference tests of the Cournot model," Econometrica, 81(6), 2351-2379.

Castillo, M. and P. Cross. 2008. "Of Mice and Men: Within Gender Variation in Strategic Behavior," Games and Economic Behavior, vol. 64, no. 2, pp. 421-32.

Charness, G. and M. Rabin. 2002. "Understanding Social Preferences with Simple Tests," Quarterly Journal of Economics, vol. 117, no. 3, pp. 817-69.

Chavas, J.-P. and T. Cox. 1993. "On Generalized Revealed Preference Analysis," Quarterly Journal of Economics, 108(2), 493-506.

Chen, M., V. Lakshminarayanan, and L. Santos. 2006. "How Basic Are Behavioral Biases? Evidence from Capuchin Monkey Trading Behavior." Journal of Political Economy, 114(3), 517-37.

Cherchye, L., B. De Rock, and F. Vermeulen. 2007. "The Collective Model of Household Consumption: A Nonparametric Characterization," Econometrica, 75(2), 553-74.

Choi, S., R. Fisman, D. Gale, and S. Kariv. 2007. "Consistency and Heterogeneity of Individual Behavior under Uncertainty," American Economic Review, 97(5), 1921-38.

Cox, J., 1997. "On Testing the Utility Hypothesis," The Economic Journal, 107(443), pp. $1054-1078$.

Crawford, I. 2010. "Habits Revealed," Review of Economic Studies, 77(4), 1382-1402.

Debreu, G. 1954. "Representation of a preference ordering by a numerical function." In Decision Prfocesses, ed. R. Thrall, C. Coombes and R. Davis. New York: Wiley

Debreu, G. 1964. "Continuity properties of Paretian utility," International Economic Review, 5(3), 285-293.

Demuynck T. 2009. "A general extension result with applications to convexity, homotheticity and monotonicity," Mathematical Social Sciences, 57(1), 96-109.

Dufwenberg, M., P. Heidhues, G. Kirchsteiger, F. Ridel, and J. Sobel. 2011. "OtherRegarding Preferences in General Equilibrium," Review of Economic Studies, 78, 613639. 
Famulari, M. 1995. "A Household-Based, Nonparametric Test of Demand Theory," Review of Economics and Statistics, 77(2), 372-82.

Fehr, E. and K. Schmidt. 1999. "A Theory of Fairness, Competition, and Cooperation," Quarterly Journal of Economics, Vol. 114, No. 3, pp. 817-868.

Fisman, R., S. Kariv, D. Markovits. 2007. "Individual Preferences for Giving," American Economic Review, vol. 97, no. 5, pp. 1858-76.

Forges, F. and E. Minelli. 2009. "Afriat's Theorem for General Budget Sets," Journal of Economic Theory, 144(1), 135-45.

Gilboa, I. and D. Schmeidler. 2003. "A Derivation of Expected Utility Maximization in the Context of a Game," Games and Economic Behavior, 44(1), pp. 184-194.

Guth, W., R. Schmittberger and B. Schwartz. 1982. "An Experimental Analysis of Ultimatum Bargaining." Journal of Games and Economic Behavior, 3(4), pp. 367-88.

Harbaugh, W., K. Krause, and T. Berry. 2001. "Garp for Kids: On the Development of Rational Choice Behavior," American Economic Review, 91(5), 1539-45.

Hossain, T., and Okui, R. 2013. "The binarized scoring rule," Review of Economic Studies, 80(3), 984-1001.

Kagel, J., R. Battalio, and L. Green. 1995. Economic choice theory: An experimental analysis of animal behavior, Cambridge; New York and Melbourne: Cambridge University Press.

Kagel, J., C. Kim and D. Moser. 1996. "Fairness in Ultimatum Games with Asymmetric Information and Asymmetric Payoffs," Games and Economic Behavior, vol. 13, no. 1, pp. 100-110.

Manski, C.F. 2002. "Identification of decision rules in experiments on simple games of proposal and response," European Economic Review, 46(4-5), 880-91.

Manski, C.F. 2004. "Measuring expectations," Econometrica, 72(5), 1329-76.

Manski, C.F. and C. Neri. 2013. "First- and second-order subjective expectations in strategic decision-making: Experimental evidence," Games and Economic Behavior, 81, pp.232-54.

Matzkin, R. 1991. "Axioms of Revealed Preference for Nonlinear Choice Sets," Econometrica, 59(6), 1779-86. 
Richter, M. K. 1966. "Revealed preference theory," Econometrica, 34(3), 635.

Roth, A. 1995. Bargaining Experiments. In: Kagel, J., Roth, A. (Eds.), Handbook of Experimental Economics, Princeton, NJ, Princeton University Press.

Roth, A., Prasnikar V., Okunofujiwara, M., S. Zamir, S. 1991. "Bargaining and Market Behavior in Jerusalem, Ljubljana, Pittsburgh, and Tokyo: An Experimental Study," American Economic Review, 81, 1068-1095.

Samuelson, P., 1938. "The Empirical Implications of Utility Analysis," Econometrica, vol. 6, no. 4, pp. 344-356.

Schotter, A. and I. Treviño. 2014. "Belief Elicitation in the Laboratory," Annual Review of Economics, Vol. 6, pp. 103-28.

Sippel, R. 1997. "An Experiment on the Pure Theory of Consumer's Behaviour," Economic Journal, 107(444): 1431-44.

Sprumont, Y. 2000. "On the Testable Implications of Collective Choice Theories," Journal of Economic Theory, 93(2), 205-32.

Szpilrajn, E. 1930. "Sur l'extension de l'ordre partiel," Fundamenta mathematicae 1.16: 386-389.

Varian, H. 1992.Microeconomic Analysis, 3rd ed., New York: Norton. 
Appendix C (For Online Publication Only): Experimental Instructions

\section{C.1: Decision Task}

\section{INSTRUCTIONS}

\section{Welcome}

This is an experiment about decision making. You will be paid for your participation. The amount of money you earn depends on your decisions and the decisions of others. If you make good decisions, you could earn a considerable amount of money. The experiment should take about an hour. At the end of the experiment you will be paid privately and in cash for your decisions. A research foundation has provided the funds for this experiment.

\section{Thanks For Showing Up}

Just for being willing to participate, you will automatically earn $\$ 8$. Whatever you earn in the rest of the session will be in addition to this $\$ 8$.

\section{Your Identity}

You will never be asked to reveal your identity to anyone during the course of the experiment. Your name will never be recorded. Neither the experimenters nor anyone else in the room will be able to link you to any of your decisions. In order to keep your decisions private, please do not reveal your choices to any other participant.

\section{Claim Check}

At the top of this page is a number on a yellow piece of paper. This is your Claim Check. Each participant has a different number. Please verify that the number on your Claim Check is the same as the Claim Check Number on the top of page X.

You will present your Claim Check to an assistant at the end of the experiment to receive your cash payment.

Please remove your claim check now and put it an a safe place.

\section{THIS EXPERIMENT}

You and the other person will be paired randomly and anonymously. No one will know the identity of the other person in your pair. In this experiment you are asked to make a series of choices about how to allocate a sum of money between yourself and one other person in the room.

The task of each pair is to divide from $\$ 0$ to up to $\$ 70$ between the two of you. How much money you end up with at the end of the experiment depends on the decisions both people in 
the pair make.

In each pair, one person will be the Proposer and the other will be the Responder. Your role will be determined at the end of the session, hence you must understand both roles to make good choices. In each of the series of choices the Proposer chooses a Proposal Rule. A Proposal Rule determines how much money will go to the Proposer and how much will go to the Responder. Given the Proposal Rule the Proposer chooses, the Responder responds by choosing whether to Accept or Reject the proposal. If the Responder responds with Accept, then the Proposer and the Responder receive the sums of money determined by the chosen Proposal Rule. If the Responder responds with Reject, then the Proposer and the Responder both receive nothing.

IMPORTANT: The Proposer chooses the Proposal Rule without knowing whether the Responder will respond by Accepting or Rejecting the Proposal Rule.

The Proposal Rules must be chosen from a table like this:

\begin{tabular}{|c|l|ll|}
\hline $\begin{array}{c}\text { Proposer chooses Proposal Rule } \\
\text { by circling one letter in this column }\end{array}$ & \multicolumn{4}{|c|}{ Proposal Rules to choose from: } \\
\hline a & Proposer gets $\$ 59$ and Responder gets & $\$ 1$ \\
\hline b & Proposer gets $\$ 55$ and Responder gets & $\$ 5$ \\
\hline c & Proposer gets $\$ 50$ and Responder gets & $\$ 10$ \\
\hline d & Proposer gets $\$ 45$ and Responder gets & $\$ 15$ \\
\hline e & Proposer gets $\$ 40$ and Responder gets & $\$ 20$ \\
\hline f & Proposer gets $\$ 35$ and Responder gets & $\$ 25$ \\
\hline g & Proposer gets $\$ 30$ and Responder gets & $\$ 30$ \\
\hline h & Proposer gets $\$ 25$ and Responder gets & $\$ 35$ \\
\hline i & Proposer gets $\$ 20$ and Responder gets & $\$ 40$ \\
\hline j & Proposer gets $\$ 15$ and Responder gets & $\$ 45$ \\
\hline k & Proposer gets $\$ 10$ and Responder gets & $\$ 50$ \\
\hline l & Proposer gets & $\$ 5$ and Responder gets & $\$ 55$ \\
\hline m & Proposer gets $\$ 1$ and Responder gets & $\$ 59$ \\
\hline
\end{tabular}

So, out of the thirteen Proposal Rules in the table, the Proposer must choose only one of them.

Given that the Proposer has selected a Proposal Rule, then the Responder responds by Accepting or Rejecting the proposal. 
However, the Responder must respond before finding out the Proposal Rule chosen by the Proposer. So, for all possible Proposal Rules, the Responder must decide whether to Accept or Reject.

The Responder will make the thirteen choices from a table like this:

\begin{tabular}{|c|c|c|c|c|c|c|}
\hline & \multicolumn{4}{|c|}{$\begin{array}{l}\text { If you are the Responder and the } \\
\text { Proposer chooses Proposal Rule.. }\end{array}$} & \multicolumn{2}{|c|}{$\begin{array}{c}\text {...then I choose to } \\
\text { (circle one for Proposal Rule): }\end{array}$} \\
\hline $\mathbf{a}$ & Proposer gets & $\$ 59$ & and Responder gets & $\$ 1$ & Accept & Reject \\
\hline b & Proposer gets & $\$ 55$ & and Responder gets & $\$ 5$ & Accept & Reject \\
\hline c & Proposer gets & $\$ 50$ & and Responder gets & $\$ 10$ & Accept & Reject \\
\hline d & Proposer gets & $\$ 45$ & and Responder gets & $\$ 15$ & Accept & Reject \\
\hline $\mathbf{e}$ & Proposer gets & $\$ 40$ & and Responder gets & $\$ 20$ & Accept & Reject \\
\hline f & Proposer gets & $\$ 35$ & and Responder gets & $\$ 25$ & Accept & Reject \\
\hline g & Proposer gets & $\$ 30$ & and Responder gets & $\$ 30$ & Accept & Reject \\
\hline $\mathbf{h}$ & Proposer gets & $\$ 25$ & and Responder gets & $\$ 35$ & Accept & Reject \\
\hline $\mathbf{i}$ & Proposer gets & $\$ 20$ & and Responder gets & $\$ 40$ & Accept & Reject \\
\hline $\mathbf{j}$ & Proposer gets & $\$ 15$ & and Responder gets & $\$ 45$ & Accept & Reject \\
\hline k & Proposer gets & $\$ 10$ & and Responder gets & $\$ 50$ & Accept & Reject \\
\hline 1 & Proposer gets & $\$ 5$ & and Responder gets & $\$ 55$ & Accept & Reject \\
\hline $\mathbf{m}$ & Proposer gets & $\$ 1$ & and Responder gets & $\$ 60$ & Accept & Reject \\
\hline
\end{tabular}

\section{EXAMPLES}

We now consider some examples.

Example One: Suppose the Proposer circles Proposal Rule k: "Proposer gets $\$ 10$ and Responder gets \$50." Suppose also that the Responder circles Accept on line k. Then, since the Responder chose to Accept, the Proposer receives $\$ 10$ and the Responder receives $\$ 50$.

Example Two: Suppose the Proposer circles Proposal Rule d: "Proposer gets $\$ 45$ and Responder gets $\$ 15$." Suppose also that the Responder circles Accept on line d. Then, since the Responder chose to Accept, the Proposer receives $\$ 45$ and the Responder receives $\$ 15$.

Example Three: Suppose the Proposer circles Proposal Rule g: "Proposer gets $\$ 30$ and Responder gets $\$ 30$." Suppose also that the Responder circles Reject on line g. Then, since the Responder chose to Reject, the Proposer receives $\$ 0$ and the Responder receive $\$ 0$. 


\section{EXERCISES}

While calculating payoffs seems easy, it is important that everyone understand how to calculate payoffs of both the Proposer and the Responder. So, below we ask you to calculate the payoffs of both players for some specific examples. After you have finished, we will go over the correct answers together.

CASE OnE: Suppose the Proposer chooses:

f 1 Proposer gets $\$ 35$ and Responder gets $\$ 25$

and for Proposal rule $f$ the Responder chooses:

\begin{tabular}{|l|lll|ll|}
\hline f & Proposer gets $\$ 35$ and Responder gets $\$ 25$ & Accept Reject \\
\hline
\end{tabular}

Payoff for the Proposer is $\$_{-----.}$Payoff for the Responder is $\$$

CASE Two: Suppose the Proposer chooses:

e $\quad$ Proposer gets $\$ 10$ and Responder gets $\$ 28$

and for Proposal rule $f$ the Responder chooses:

\begin{tabular}{|l|lll|ll|}
\hline e & Proposer gets $\$ 10$ and Responder gets $\$ 28$ & Accept Reject \\
\hline
\end{tabular}

Payoff for the Proposer is $\$_{-----}$. Payoff for the Responder is $\$_{-----}$.

Case Three: Suppose the Proposer chooses:

\begin{tabular}{|l|lll}
\hline b & Proposer gets $\$ 44$ and Responder gets $\$ 2$ \\
\hline
\end{tabular}

and for Proposal rule $f$ the Responder chooses:

\begin{tabular}{|l|lll|ll|}
\hline $\mathbf{b}$ & Proposer gets $\$ 44$ and Responder gets $\$ 2$ & Accept & Reject \\
\hline
\end{tabular}

Payoff for the Proposer is $\$_{-----.}$Payoff for the Responder is $\$_{-----}$. 


\section{YOUR ROLE}

You will be randomly assigned either to the role of the Proposer or to the role of the Responder. After you are assigned a role, you will be randomly matched with another person in the room, and your decision for only the role assigned to you will be carried out. One of your decisions will be chosen randomly and carried out.

\section{IMPORTANT}

You must make all your decisions before you know to which role you will be assigned.

Since you won't know to which role you will be assigned until the very end, you must make decisions for both roles. After all decisions are made, there is a $50 \%$ chance you will be assigned the Proposer role and a 50\% chance you will be assigned the Responder role.

\section{HOW THE PAIRINGS ARE MADE}

Attached are decision forms for the Proposer and the Responder. Complete all forms, imagining as being chosen for each role. Place the completed forms and instructions back on the envelope.

After you have finished making your decisions, you will put the completed forms in your envelope. We will collect the envelopes, shuffle them, and separate them into two piles of equal size; Pile 1 and Pile 2.

If, by chance, your envelope is in Pile 1, you will be a Proposer - we will use only your decisions in the Proposer role, and ignore your decisions in the Responder role.

If, by chance, your envelope is in Pile 2, you will be a Responder - we will use only your decisions in the Responder role, and ignore your decisions in the Proposer role.

After shuffling the envelopes in each pile again, each envelope in Pile 1, the Proposers, will be matched with an envelope in Pile 2, the Responders. This is the way each of you will be randomly paired with another person in the room. 


\section{RECEIVING YOUR PAYMENT}

After all pairings are made, we will randomly select one of the 9 possible decisions for each pairing. We will use the alternatives chosen by the Proposer and the Responder to determine the payoffs for that pair. Your payoff from the pairing will be placed in your earnings envelope with your Claim Check Number written on the outside of the envelope. You will present your Claim Check, and we will hand you your earnings envelope.

To further guard your anonymity, an assistant who was not involved in determining the payoffs, and thus has no idea what is in each envelope, will hand you your earnings envelope.

Finally, to verify that the procedures we describe are followed, a monitor, who was chosen at the beginning of the experiment, will be present during the determination of payments.

Preparing your payments will take about 15 minutes.

\section{SUMMARY}

Step 1: You will eventually assume the role of either Proposer or Responder. However, before you are randomly assigned a role, you must make choices for both the Proposer role and the Responder role.

Step 2: After your choices are made, we will randomly assign you the role of either Proposer or Responder.

Step 3: You will be randomly paired with another person in the room, who has been assigned the other role. Your choice only for the role assigned to you will be carried out with the other person in your pairing.

Step 4: For each pair, one of the 8 decisions will be chosen at random and both of your decisions will be carried out.

Step 5: Everyone will receive cash payments in private envelopes at the end of the experiment.

\section{You can begin making your decisions Good luck!}


Suppose you are the Proposer. Choose one and only one alternative from the following table. Please circle the letter of your choice from the first column.

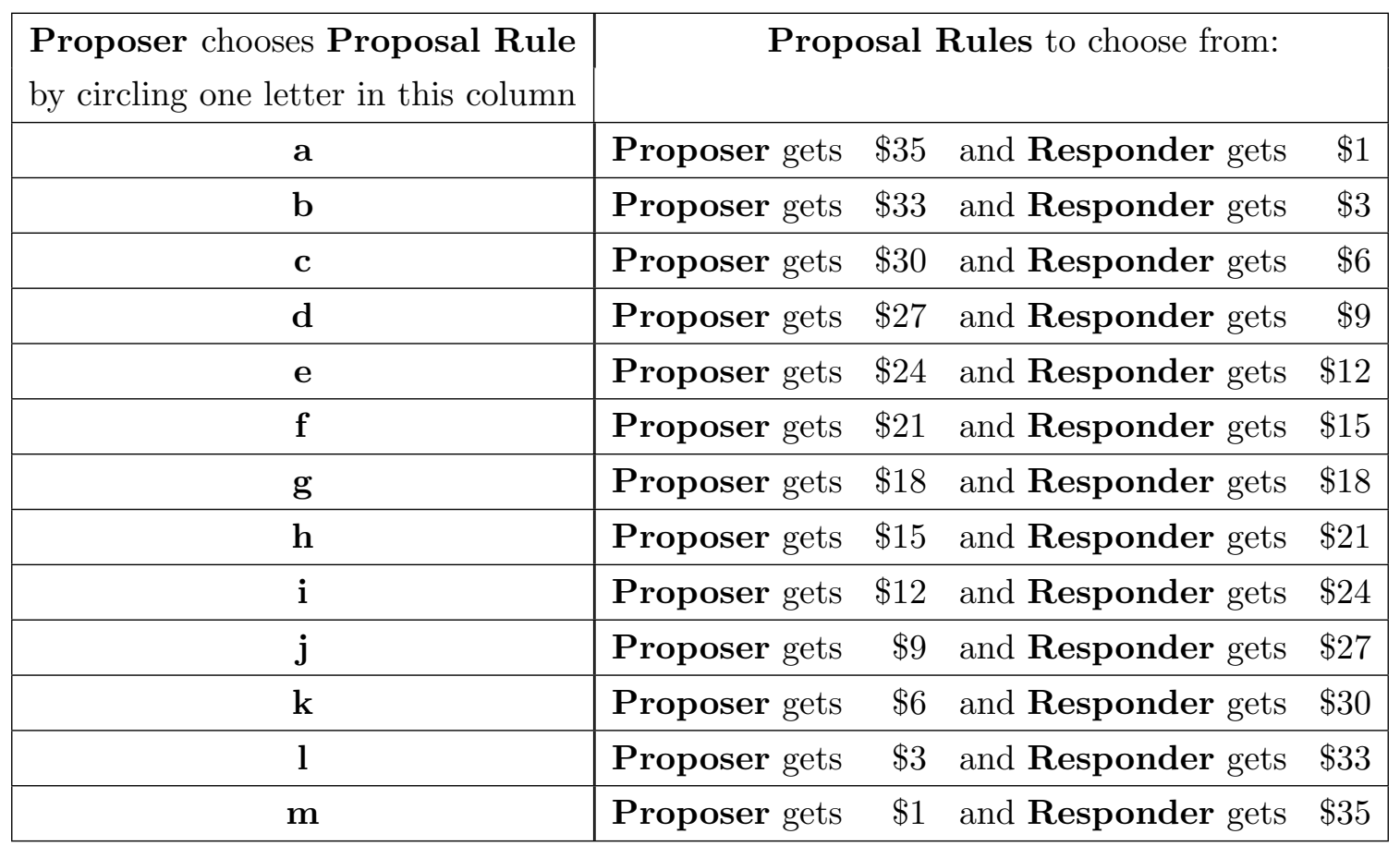




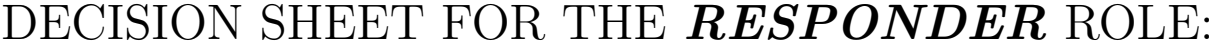

Suppose you are the Responder. Circle either Accept or Reject in the last column. Please complete the table for each possible alternative.

\begin{tabular}{|l|llll|cc|}
\hline & \multicolumn{2}{|c|}{$\begin{array}{c}\text { If you are the Responder and the } \\
\text { Proposer chooses Proposal Rule.. }\end{array}$} & \multicolumn{2}{c|}{$\begin{array}{c}\text {..then I choose to } \\
\text { (circle one for Proposal Rule): }\end{array}$} \\
\hline $\mathbf{a}$ & Proposer gets & $\$ 35$ & and Responder gets & $\$ 1$ & Accept & Reject \\
\hline b & Proposer gets & $\$ 33$ & and Responder gets & $\$ 3$ & Accept & Reject \\
\hline $\mathbf{c}$ & Proposer gets & $\$ 30$ & and Responder gets & $\$ 6$ & Accept & Reject \\
\hline $\mathbf{d}$ & Proposer gets & $\$ 27$ & and Responder gets & $\$ 9$ & Accept & Reject \\
\hline $\mathbf{e}$ & Proposer gets & $\$ 24$ & and Responder gets & $\$ 12$ & Accept & Reject \\
\hline $\mathbf{f}$ & Proposer gets & $\$ 21$ & and Responder gets & $\$ 15$ & Accept & Reject \\
\hline $\mathbf{g}$ & Proposer gets & $\$ 18$ & and Responder gets & $\$ 18$ & Accept & Reject \\
\hline $\mathbf{h}$ & Proposer gets & $\$ 15$ & and Responder gets & $\$ 21$ & Accept & Reject \\
\hline $\mathbf{i}$ & Proposer gets & $\$ 12$ & and Responder gets & $\$ 24$ & Accept & Reject \\
\hline $\mathbf{j}$ & Proposer gets & $\$ 9$ & and Responder gets & $\$ 27$ & Accept & Reject \\
\hline $\mathbf{k}$ & Proposer gets & $\$ 6$ & and Responder gets & $\$ 30$ & Accept & Reject \\
\hline $\mathbf{l}$ & Proposer gets & $\$ 3$ & and Responder gets & $\$ 33$ & Accept & Reject \\
\hline $\mathbf{m}$ & Proposer gets & $\$ 1$ & and Responder gets & $\$ 35$ & Accept & Reject \\
\hline
\end{tabular}




\section{DECISION SHEET FOR THE PROPOSER ROLE:}

Suppose you are the Proposer. Choose one and only one alternative from the following table. Please circle the letter of your choice from the first column.

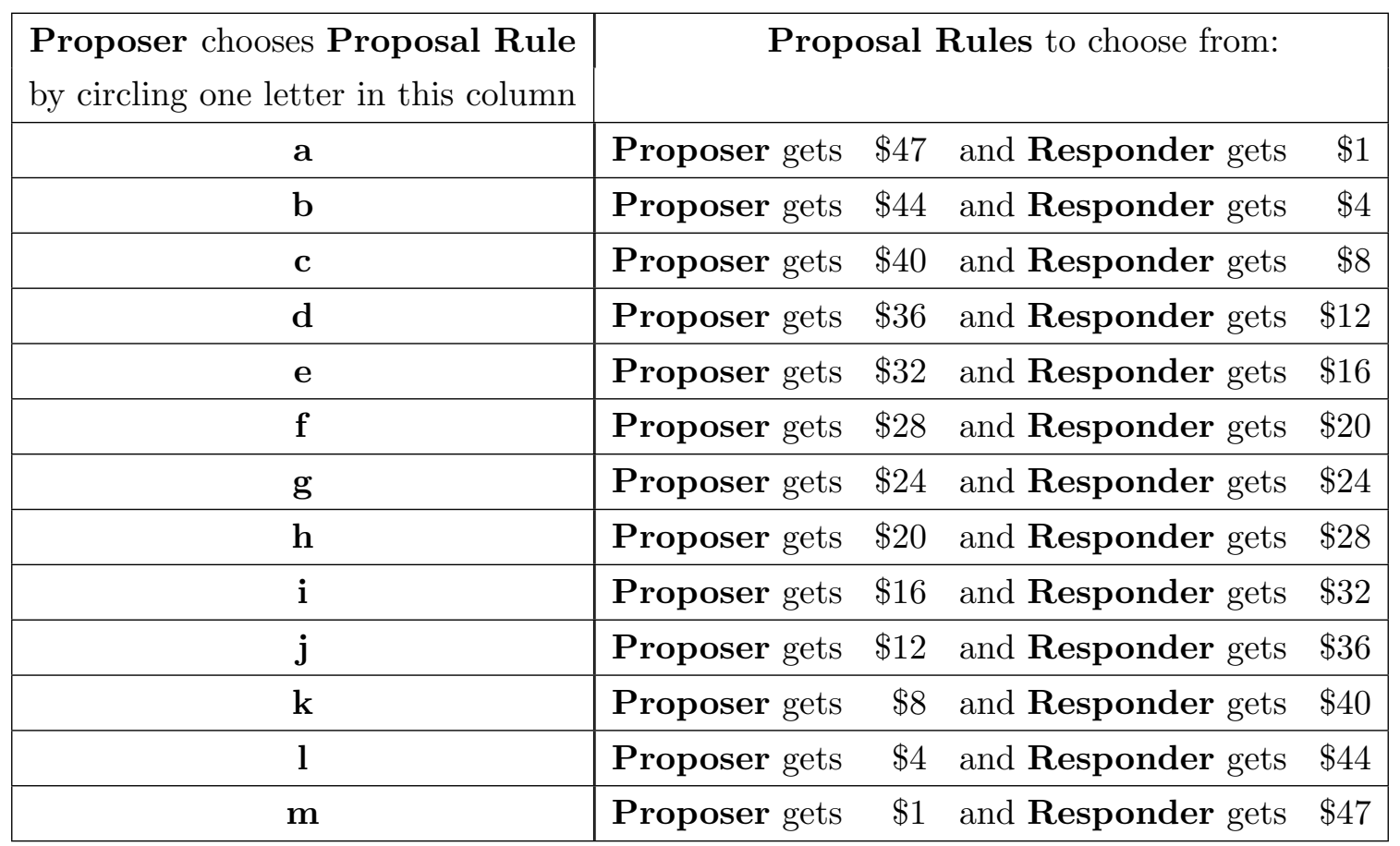




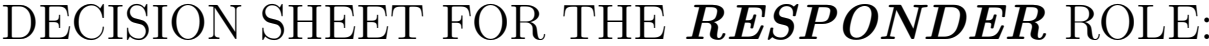

Suppose you are the Responder. Circle either Accept or Reject in the last column. Please complete the table for each possible alternative.

\begin{tabular}{|l|llll|cc|}
\hline & \multicolumn{2}{|c|}{$\begin{array}{c}\text { If you are the Responder and the } \\
\text { Proposer chooses Proposal Rule.. }\end{array}$} & \multicolumn{2}{c|}{$\begin{array}{c}\text {..then I choose to } \\
\text { (circle one for Proposal Rule): }\end{array}$} \\
\hline $\mathbf{a}$ & Proposer gets & $\$ 47$ & and Responder gets & $\$ 1$ & Accept & Reject \\
\hline b & Proposer gets & $\$ 44$ & and Responder gets & $\$ 4$ & Accept & Reject \\
\hline $\mathbf{c}$ & Proposer gets & $\$ 40$ & and Responder gets & $\$ 8$ & Accept & Reject \\
\hline $\mathbf{d}$ & Proposer gets & $\$ 36$ & and Responder gets & $\$ 12$ & Accept & Reject \\
\hline $\mathbf{e}$ & Proposer gets & $\$ 32$ & and Responder gets & $\$ 16$ & Accept & Reject \\
\hline $\mathbf{f}$ & Proposer gets & $\$ 28$ & and Responder gets & $\$ 20$ & Accept & Reject \\
\hline $\mathbf{g}$ & Proposer gets & $\$ 24$ & and Responder gets & $\$ 24$ & Accept & Reject \\
\hline $\mathbf{h}$ & Proposer gets & $\$ 20$ & and Responder gets & $\$ 28$ & Accept & Reject \\
\hline $\mathbf{i}$ & Proposer gets & $\$ 16$ & and Responder gets & $\$ 32$ & Accept & Reject \\
\hline $\mathbf{j}$ & Proposer gets & $\$ 12$ & and Responder gets & $\$ 36$ & Accept & Reject \\
\hline $\mathbf{k}$ & Proposer gets & $\$ 8$ & and Responder gets & $\$ 40$ & Accept & Reject \\
\hline $\mathbf{l}$ & Proposer gets & $\$ 4$ & and Responder gets & $\$ 44$ & Accept & Reject \\
\hline $\mathbf{m}$ & Proposer gets & $\$ 1$ & and Responder gets & $\$ 47$ & Accept & Reject \\
\hline
\end{tabular}


Suppose you are the Proposer. Choose one and only one alternative from the following table. Please circle the letter of your choice from the first column.

\begin{tabular}{|c|c|c|c|c|}
\hline Proposer chooses Proposal Rule & Prop & osal & Rules to choose from: & \\
\hline $\mathbf{a}$ & Proposer gets & $\$ 59$ & and Responder gets & $\$ 1$ \\
\hline b & Proposer gets & $\$ 55$ & and Responder gets & $\$ 5$ \\
\hline c & Proposer gets & $\$ 50$ & and Responder gets & $\$ 10$ \\
\hline d & Proposer gets & $\$ 45$ & and Responder gets & $\$ 15$ \\
\hline e & Proposer gets & $\$ 40$ & and Responder gets & $\$ 20$ \\
\hline f & Proposer gets & $\$ 35$ & and Responder gets & $\$ 25$ \\
\hline g & Proposer gets & $\$ 30$ & and Responder gets & $\$ 30$ \\
\hline $\mathbf{h}$ & Proposer gets & $\$ 25$ & and Responder gets & $\$ 35$ \\
\hline i & Proposer gets & $\$ 20$ & and Responder gets & $\$ 40$ \\
\hline $\mathbf{j}$ & Proposer gets & $\$ 15$ & and Responder gets & $\$ 45$ \\
\hline $\mathrm{k}$ & Proposer gets & $\$ 10$ & and Responder gets & $\$ 50$ \\
\hline 1 & Proposer gets & $\$ 5$ & and Responder gets & $\$ 55$ \\
\hline $\mathbf{m}$ & Proposer gets & $\$ 1$ & and Responder gets & $\$ 59$ \\
\hline
\end{tabular}




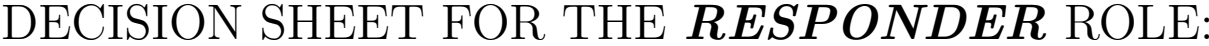

Suppose you are the Responder. Circle either Accept or Reject in the last column. Please complete the table for each possible alternative.

\begin{tabular}{|c|c|c|c|c|c|}
\hline & \multicolumn{3}{|c|}{$\begin{array}{l}\text { If you are the Responder and the } \\
\text { Proposer chooses Proposal Rule.. }\end{array}$} & \multicolumn{2}{|c|}{$\begin{array}{c}\text {...then I choose to } \\
\text { (circle one for Proposal Rule): }\end{array}$} \\
\hline $\mathbf{a}$ & Proposer gets $\$ 59$ & and Responder gets & $\$ 1$ & Accept & Reject \\
\hline $\mathbf{b}$ & Proposer gets $\$ 55$ & and Responder gets & $\$ 5$ & Accept & Reject \\
\hline $\mathbf{c}$ & Proposer gets $\$ 50$ & and Responder gets & $\$ 10$ & Accept & Reject \\
\hline$d$ & Proposer gets $\$ 45$ & and Responder gets & $\$ 15$ & Accept & Reject \\
\hline e & Proposer gets $\$ 40$ & and Responder gets & $\$ 20$ & Accept & Reject \\
\hline $\mathrm{f}$ & Proposer gets $\$ 35$ & and Responder gets & $\$ 25$ & Accept & Reject \\
\hline $\mathrm{g}$ & Proposer gets $\$ 30$ & and Responder gets & $\$ 30$ & Accept & Reject \\
\hline $\mathbf{h}$ & Proposer gets $\$ 25$ & and Responder gets & $\$ 35$ & Accept & Reject \\
\hline i & Proposer gets $\$ 20$ & and Responder gets & $\$ 40$ & Accept & Reject \\
\hline j & Proposer gets $\$ 15$ & and Responder gets & $\$ 45$ & Accept & Reject \\
\hline $\mathrm{k}$ & Proposer gets $\$ 10$ & and Responder gets & $\$ 50$ & Accept & Reject \\
\hline 1 & Proposer gets & and Responder gets & $\$ 55$ & Accept & Reject \\
\hline $\mathbf{m}$ & Proposer gets & and Responder gets & $\$ 59$ & Accept & Reject \\
\hline
\end{tabular}


Suppose you are the Proposer. Choose one and only one alternative from the following table. Please circle the letter of your choice from the first column.

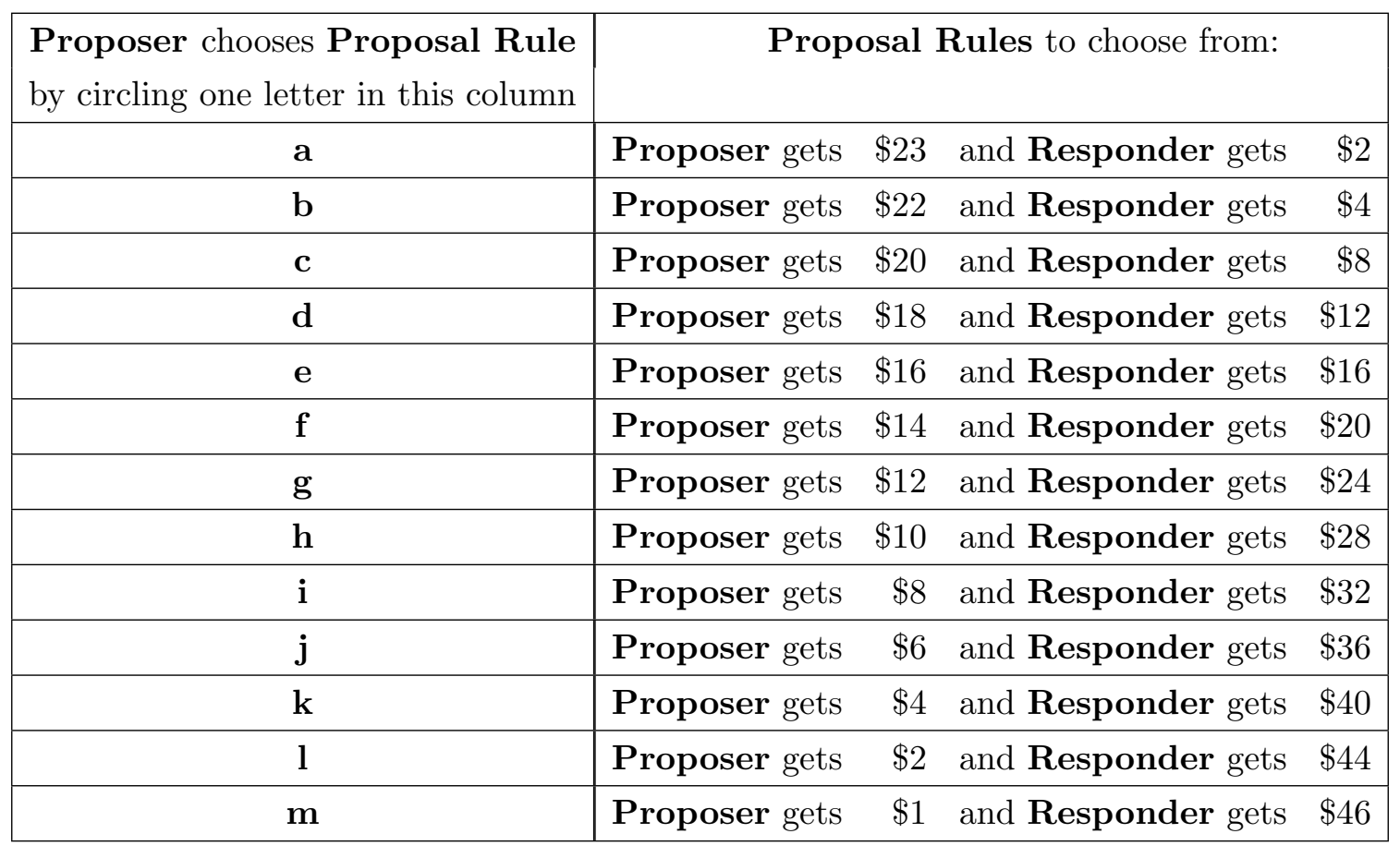




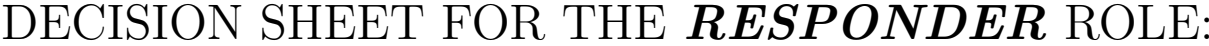

Suppose you are the Responder. Circle either Accept or Reject in the last column. Please complete the table for each possible alternative.

\begin{tabular}{|l|llll|cc|}
\hline & \multicolumn{2}{|c|}{$\begin{array}{c}\text { If you are the Responder and the } \\
\text { Proposer chooses Proposal Rule.. }\end{array}$} & \multicolumn{2}{c|}{$\begin{array}{c}\text {..then I choose to } \\
\text { (circle one for Proposal Rule): }\end{array}$} \\
\hline $\mathbf{a}$ & Proposer gets & $\$ 23$ and Responder gets & $\$ 2$ & Accept & Reject \\
\hline b & Proposer gets & $\$ 22$ & and Responder gets & $\$ 4$ & Accept & Reject \\
\hline $\mathbf{c}$ & Proposer gets & $\$ 20$ & and Responder gets & $\$ 8$ & Accept & Reject \\
\hline $\mathbf{d}$ & Proposer gets & $\$ 18$ & and Responder gets & $\$ 12$ & Accept & Reject \\
\hline $\mathbf{e}$ & Proposer gets & $\$ 16$ & and Responder gets & $\$ 16$ & Accept & Reject \\
\hline $\mathbf{f}$ & Proposer gets & $\$ 14$ & and Responder gets & $\$ 20$ & Accept & Reject \\
\hline $\mathbf{g}$ & Proposer gets & $\$ 12$ & and Responder gets & $\$ 24$ & Accept & Reject \\
\hline $\mathbf{h}$ & Proposer gets & $\$ 10$ & and Responder gets & $\$ 28$ & Accept & Reject \\
\hline $\mathbf{i}$ & Proposer gets & $\$ 8$ & and Responder gets & $\$ 32$ & Accept & Reject \\
\hline $\mathbf{j}$ & Proposer gets & $\$ 6$ & and Responder gets & $\$ 36$ & Accept & Reject \\
\hline $\mathbf{k}$ & Proposer gets & $\$ 4$ & and Responder gets & $\$ 40$ & Accept & Reject \\
\hline $\mathbf{l}$ & Proposer gets & $\$ 2$ & and Responder gets & $\$ 44$ & Accept & Reject \\
\hline $\mathbf{m}$ & Proposer gets & $\$ 1$ & and Responder gets & $\$ 46$ & Accept & Reject \\
\hline
\end{tabular}


Suppose you are the Proposer. Choose one and only one alternative from the following table. Please circle the letter of your choice from the first column.

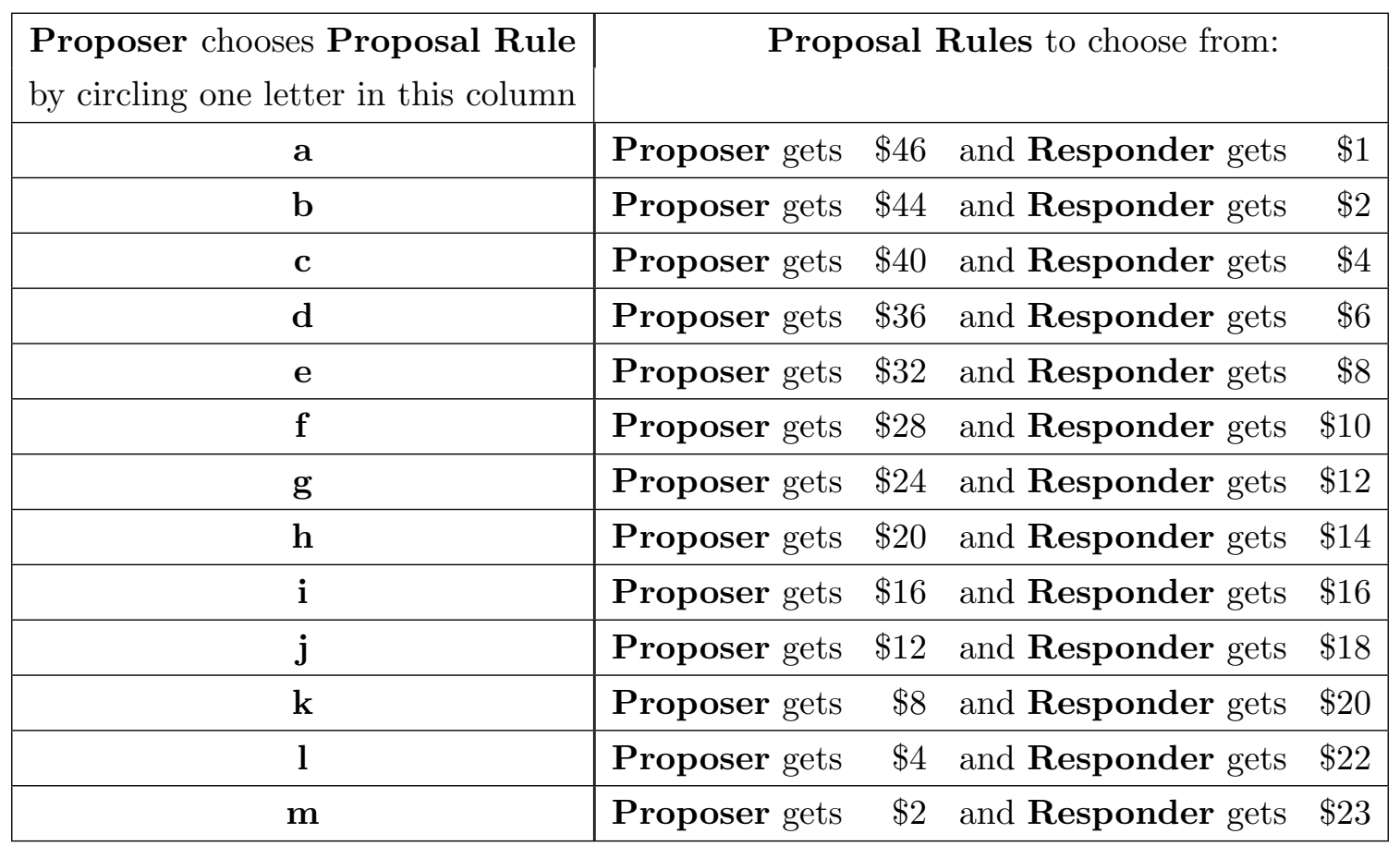




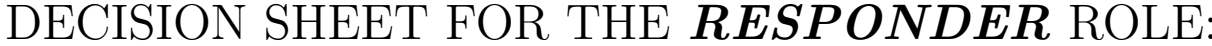

Suppose you are the Responder. Circle either Accept or Reject in the last column. Please complete the table for each possible alternative.

\begin{tabular}{|l|llll|cc|}
\hline & \multicolumn{2}{|c|}{$\begin{array}{c}\text { If you are the Responder and the } \\
\text { Proposer chooses Proposal Rule.. }\end{array}$} & \multicolumn{2}{c|}{$\begin{array}{c}\text {..then I choose to } \\
\text { (circle one for Proposal Rule): }\end{array}$} \\
\hline $\mathbf{a}$ & Proposer gets & $\$ 46$ & and Responder gets & $\$ 1$ & Accept & Reject \\
\hline b & Proposer gets & $\$ 44$ & and Responder gets & $\$ 2$ & Accept & Reject \\
\hline $\mathbf{c}$ & Proposer gets & $\$ 40$ & and Responder gets & $\$ 4$ & Accept & Reject \\
\hline $\mathbf{d}$ & Proposer gets & $\$ 36$ & and Responder gets & $\$ 6$ & Accept & Reject \\
\hline $\mathbf{e}$ & Proposer gets & $\$ 32$ & and Responder gets & $\$ 8$ & Accept & Reject \\
\hline $\mathbf{f}$ & Proposer gets & $\$ 28$ & and Responder gets & $\$ 10$ & Accept & Reject \\
\hline $\mathbf{g}$ & Proposer gets & $\$ 24$ & and Responder gets & $\$ 12$ & Accept & Reject \\
\hline $\mathbf{h}$ & Proposer gets & $\$ 20$ & and Responder gets & $\$ 14$ & Accept & Reject \\
\hline $\mathbf{i}$ & Proposer gets & $\$ 16$ & and Responder gets & $\$ 16$ & Accept & Reject \\
\hline $\mathbf{j}$ & Proposer gets & $\$ 12$ & and Responder gets & $\$ 18$ & Accept & Reject \\
\hline $\mathbf{k}$ & Proposer gets & $\$ 8$ & and Responder gets & $\$ 20$ & Accept & Reject \\
\hline $\mathbf{l}$ & Proposer gets & $\$ 4$ & and Responder gets & $\$ 22$ & Accept & Reject \\
\hline $\mathbf{m}$ & Proposer gets & $\$ 2$ & and Responder gets & $\$ 23$ & Accept & Reject \\
\hline
\end{tabular}


Suppose you are the Proposer. Choose one and only one alternative from the following table. Please circle the letter of your choice from the first column.

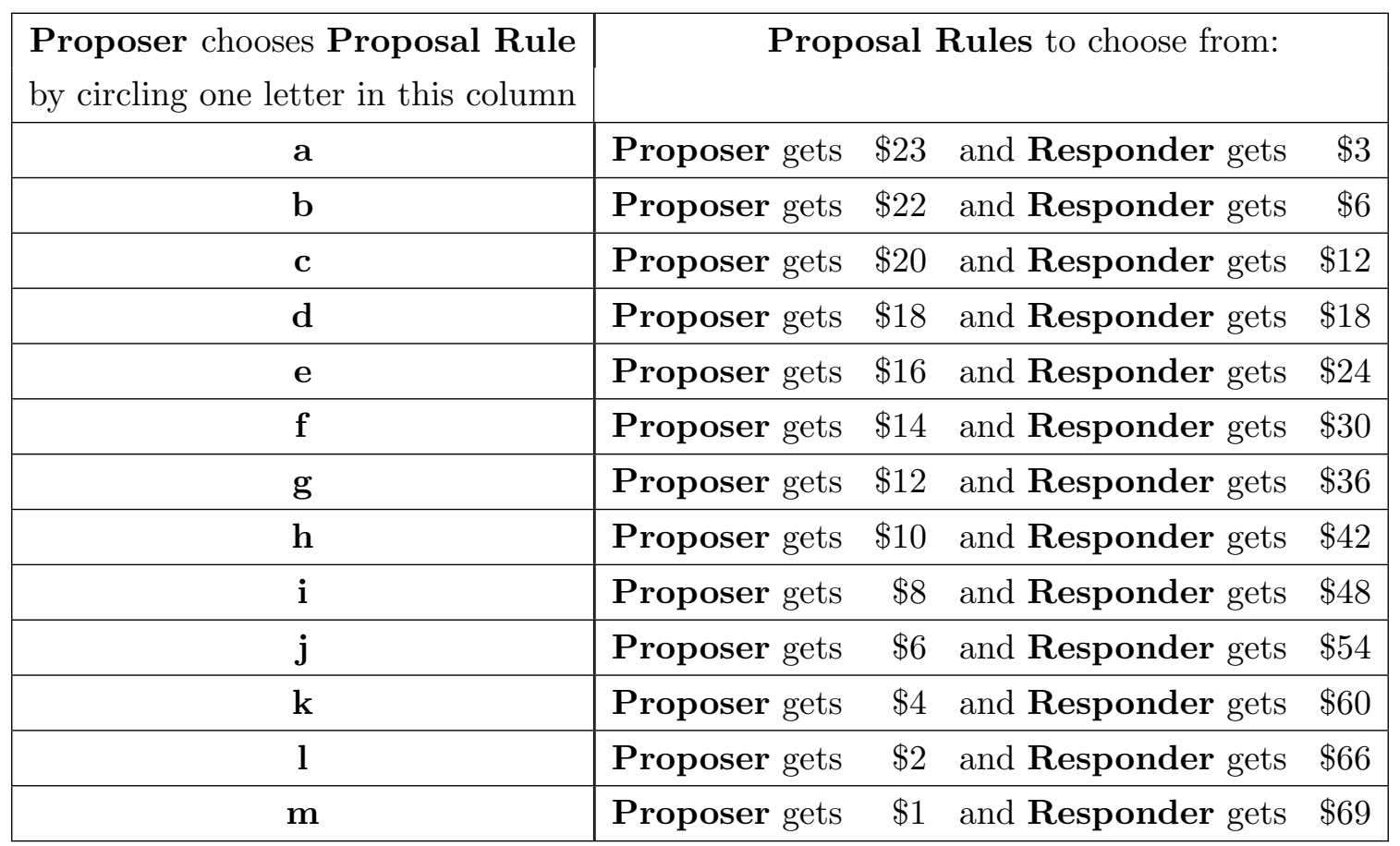




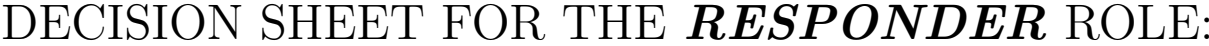

Suppose you are the Responder. Circle either Accept or Reject in the last column. Please complete the table for each possible alternative.

\begin{tabular}{|l|llll|cc|}
\hline & \multicolumn{2}{|c|}{$\begin{array}{c}\text { If you are the Responder and the } \\
\text { Proposer chooses Proposal Rule.. }\end{array}$} & \multicolumn{2}{c|}{$\begin{array}{c}\text {..then I choose to } \\
\text { (circle one for Proposal Rule): }\end{array}$} \\
\hline $\mathbf{a}$ & Proposer gets & $\$ 23$ and Responder gets & $\$ 3$ & Accept & Reject \\
\hline b & Proposer gets & $\$ 22$ & and Responder gets & $\$ 6$ & Accept & Reject \\
\hline $\mathbf{c}$ & Proposer gets & $\$ 20$ & and Responder gets & $\$ 12$ & Accept & Reject \\
\hline $\mathbf{d}$ & Proposer gets & $\$ 18$ & and Responder gets & $\$ 18$ & Accept & Reject \\
\hline $\mathbf{e}$ & Proposer gets & $\$ 16$ & and Responder gets & $\$ 24$ & Accept & Reject \\
\hline $\mathbf{f}$ & Proposer gets & $\$ 14$ & and Responder gets & $\$ 30$ & Accept & Reject \\
\hline $\mathbf{g}$ & Proposer gets & $\$ 12$ & and Responder gets & $\$ 36$ & Accept & Reject \\
\hline $\mathbf{h}$ & Proposer gets & $\$ 10$ & and Responder gets & $\$ 42$ & Accept & Reject \\
\hline $\mathbf{i}$ & Proposer gets & $\$ 8$ & and Responder gets & $\$ 48$ & Accept & Reject \\
\hline $\mathbf{j}$ & Proposer gets & $\$ 6$ & and Responder gets & $\$ 54$ & Accept & Reject \\
\hline $\mathbf{k}$ & Proposer gets & $\$ 4$ & and Responder gets & $\$ 60$ & Accept & Reject \\
\hline $\mathbf{l}$ & Proposer gets & $\$ 2$ & and Responder gets & $\$ 66$ & Accept & Reject \\
\hline $\mathbf{m}$ & Proposer gets & $\$ 1$ & and Responder gets & $\$ 69$ & Accept & Reject \\
\hline
\end{tabular}




\section{DECISION SHEET FOR THE PROPOSER ROLE:}

Suppose you are the Proposer. Choose one and only one alternative from the following table. Please circle the letter of your choice from the first column.

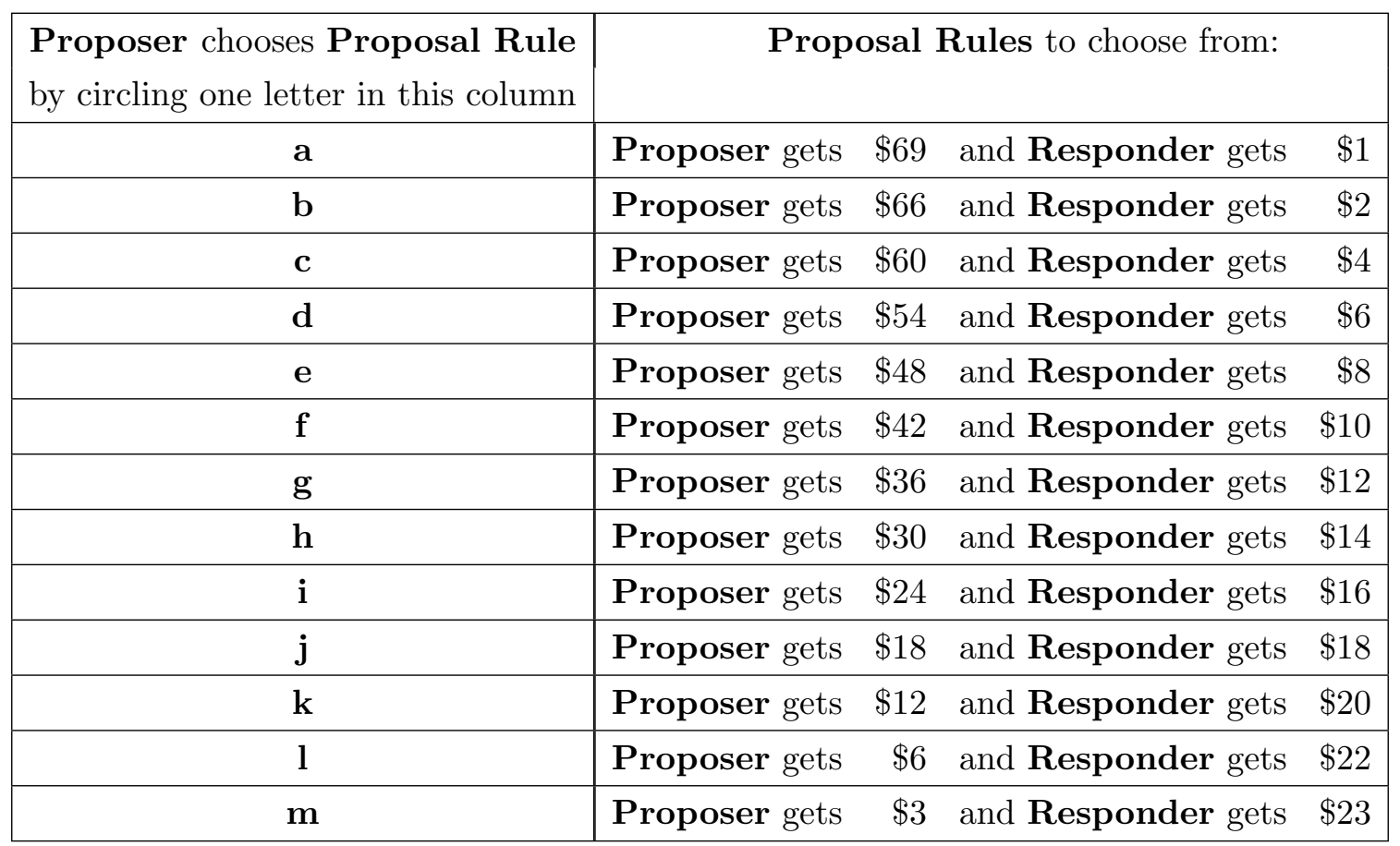




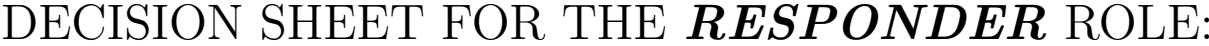

Suppose you are the Responder. Circle either Accept or Reject in the last column. Please complete the table for each possible alternative.

\begin{tabular}{|l|llll|cc|}
\hline & \multicolumn{2}{|c|}{$\begin{array}{c}\text { If you are the Responder and the } \\
\text { Proposer chooses Proposal Rule.. }\end{array}$} & \multicolumn{2}{c|}{$\begin{array}{c}\text {..then I choose to } \\
\text { (circle one for Proposal Rule): }\end{array}$} \\
\hline $\mathbf{a}$ & Proposer gets & $\$ 69$ & and Responder gets & $\$ 1$ & Accept & Reject \\
\hline b & Proposer gets & $\$ 66$ & and Responder gets & $\$ 2$ & Accept & Reject \\
\hline $\mathbf{c}$ & Proposer gets & $\$ 60$ & and Responder gets & $\$ 4$ & Accept & Reject \\
\hline $\mathbf{d}$ & Proposer gets & $\$ 54$ & and Responder gets & $\$ 6$ & Accept & Reject \\
\hline $\mathbf{e}$ & Proposer gets & $\$ 48$ & and Responder gets & $\$ 8$ & Accept & Reject \\
\hline $\mathbf{f}$ & Proposer gets & $\$ 42$ & and Responder gets & $\$ 10$ & Accept & Reject \\
\hline $\mathbf{g}$ & Proposer gets & $\$ 36$ & and Responder gets & $\$ 12$ & Accept & Reject \\
\hline $\mathbf{h}$ & Proposer gets & $\$ 30$ & and Responder gets & $\$ 14$ & Accept & Reject \\
\hline $\mathbf{i}$ & Proposer gets & $\$ 24$ & and Responder gets & $\$ 16$ & Accept & Reject \\
\hline $\mathbf{j}$ & Proposer gets & $\$ 18$ & and Responder gets & $\$ 18$ & Accept & Reject \\
\hline $\mathbf{k}$ & Proposer gets & $\$ 12$ & and Responder gets & $\$ 20$ & Accept & Reject \\
\hline $\mathbf{l}$ & Proposer gets & $\$ 6$ & and Responder gets & $\$ 22$ & Accept & Reject \\
\hline $\mathbf{m}$ & Proposer gets & $\$ 3$ & and Responder gets & $\$ 23$ & Accept & Reject \\
\hline
\end{tabular}


Suppose you are the Proposer. Choose one and only one alternative from the following table. Please circle the letter of your choice from the first column.

\begin{tabular}{|c|c|c|c|c|}
\hline \multirow{2}{*}{$\begin{array}{l}\text { Proposer chooses Proposal Rule } \\
\text { by circling one letter in this column } \\
\mathbf{a}\end{array}$} & \multicolumn{4}{|c|}{ Proposal Rules to choose from: } \\
\hline & Proposer gets & $\$ 11.50$ & and Responder gets & $\$ 2.50$ \\
\hline b & Proposer gets & $\$ 11$ & and Responder gets & $\$ 5$ \\
\hline c & Proposer gets & $\$ 10$ & and Responder gets & $\$ 10$ \\
\hline d & Proposer gets & $\$ 9$ & and Responder gets & $\$ 15$ \\
\hline e & Proposer gets & $\$ 8$ & and Responder gets & $\$ 20$ \\
\hline f & Proposer gets & $\$ 7$ & and Responder gets & $\$ 25$ \\
\hline $\mathrm{g}$ & Proposer gets & $\$ 6$ & and Responder gets & $\$ 30$ \\
\hline $\mathbf{h}$ & Proposer gets & $\$ 5$ & and Responder gets & $\$ 35$ \\
\hline i & Proposer gets & $\$ 4$ & and Responder gets & $\$ 40$ \\
\hline j & Proposer gets & $\$ 3$ & and Responder gets & $\$ 45$ \\
\hline $\mathrm{k}$ & Proposer gets & $\$ 2$ & and Responder gets & $\$ 50$ \\
\hline 1 & Proposer gets & $\$ 1$ & and Responder gets & $\$ 55$ \\
\hline $\mathbf{m}$ & Proposer gets & $\$ 0.50$ & and Responder gets & $\$ 57.50$ \\
\hline
\end{tabular}




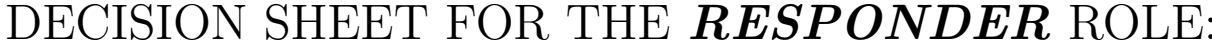

Suppose you are the Responder. Circle either Accept or Reject in the last column. Please complete the table for each possible alternative.

\begin{tabular}{|c|c|c|c|c|c|c|}
\hline & \multicolumn{4}{|c|}{$\begin{array}{l}\text { If you are the Responder and the } \\
\text { Proposer chooses Proposal Rule.. }\end{array}$} & \multicolumn{2}{|c|}{$\begin{array}{c}\text {...then I choose to } \\
\text { (circle one for Proposal Rule): }\end{array}$} \\
\hline $\mathbf{a}$ & Proposer gets & $\$ 11.50$ & and Responder gets & $\$ 2.50$ & Accept & Reject \\
\hline b & Proposer gets & $\$ 11$ & and Responder gets & $\$ 5$ & Accept & Reject \\
\hline c & Proposer gets & $\$ 10$ & and Responder gets & $\$ 10$ & Accept & Reject \\
\hline d & Proposer gets & $\$ 9$ & and Responder gets & $\$ 15$ & Accept & Reject \\
\hline $\mathbf{e}$ & Proposer gets & $\$ 8$ & and Responder gets & $\$ 20$ & Accept & Reject \\
\hline f & Proposer gets & $\$ 7$ & and Responder gets & $\$ 25$ & Accept & Reject \\
\hline $\mathrm{g}$ & Proposer gets & $\$ 6$ & and Responder gets & $\$ 30$ & Accept & Reject \\
\hline $\mathbf{h}$ & Proposer gets & $\$ 5$ & and Responder gets & $\$ 35$ & Accept & Reject \\
\hline $\mathbf{i}$ & Proposer gets & $\$ 4$ & and Responder gets & $\$ 40$ & Accept & Reject \\
\hline $\mathbf{j}$ & Proposer gets & $\$ 3$ & and Responder gets & $\$ 45$ & Accept & Reject \\
\hline k & Proposer gets & $\$ 2$ & and Responder gets & $\$ 50$ & Accept & Reject \\
\hline 1 & Proposer gets & $\$ 1$ & and Responder gets & $\$ 55$ & Accept & Reject \\
\hline $\mathbf{m}$ & Proposer gets & $\$ 0.50$ & and Responder gets & $\$ 57.50$ & Accept & Reject \\
\hline
\end{tabular}


Suppose you are the Proposer. Choose one and only one alternative from the following table. Please circle the letter of your choice from the first column.

\begin{tabular}{|c|c|c|c|c|}
\hline \multirow{2}{*}{$\begin{array}{l}\text { Proposer chooses Proposal Rule } \\
\text { by circling one letter in this column } \\
\mathbf{a}\end{array}$} & \multicolumn{4}{|c|}{ Proposal Rules to choose from: } \\
\hline & Proposer gets & $\$ 57.50$ & and Responder gets & $\$ 0.50$ \\
\hline b & Proposer gets & $\$ 55$ & and Responder gets & $\$ 1$ \\
\hline c & Proposer gets & $\$ 50$ & and Responder gets & $\$ 2$ \\
\hline d & Proposer gets & $\$ 45$ & and Responder gets & $\$ 3$ \\
\hline e & Proposer gets & $\$ 40$ & and Responder gets & $\$ 4$ \\
\hline f & Proposer gets & $\$ 35$ & and Responder gets & $\$ 5$ \\
\hline $\mathrm{g}$ & Proposer gets & $\$ 30$ & and Responder gets & $\$ 6$ \\
\hline $\mathbf{h}$ & Proposer gets & $\$ 25$ & and Responder gets & $\$ 7$ \\
\hline i & Proposer gets & $\$ 20$ & and Responder gets & $\$ 8$ \\
\hline j & Proposer gets & $\$ 15$ & and Responder gets & $\$ 9$ \\
\hline $\mathrm{k}$ & Proposer gets & $\$ 10$ & and Responder gets & $\$ 10$ \\
\hline 1 & Proposer gets & $\$ 5$ & and Responder gets & $\$ 11$ \\
\hline $\mathbf{m}$ & Proposer gets & $\$ 2.50$ & and Responder gets & $\$ 11.50$ \\
\hline
\end{tabular}




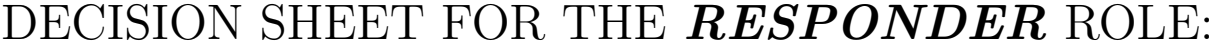

Suppose you are the Responder. Circle either Accept or Reject in the last column. Please complete the table for each possible alternative.

\begin{tabular}{|c|c|c|c|c|c|c|}
\hline & \multicolumn{4}{|c|}{$\begin{array}{l}\text { If you are the Responder and the } \\
\text { Proposer chooses Proposal Rule.. }\end{array}$} & \multicolumn{2}{|c|}{$\begin{array}{c}\text {...then I choose to } \\
\text { (circle one for Proposal Rule): }\end{array}$} \\
\hline $\mathbf{a}$ & Proposer gets & $\$ 57.50$ & and Responder gets & $\$ 0.50$ & Accept & Reject \\
\hline b & Proposer gets & $\$ 55$ & and Responder gets & $\$ 1$ & Accept & Reject \\
\hline c & Proposer gets & $\$ 50$ & and Responder gets & $\$ 2$ & Accept & Reject \\
\hline d & Proposer gets & $\$ 45$ & and Responder gets & $\$ 3$ & Accept & Reject \\
\hline $\mathbf{e}$ & Proposer gets & $\$ 40$ & and Responder gets & $\$ 4$ & Accept & Reject \\
\hline f & Proposer gets & $\$ 35$ & and Responder gets & $\$ 5$ & Accept & Reject \\
\hline $\mathrm{g}$ & Proposer gets & $\$ 30$ & and Responder gets & $\$ 6$ & Accept & Reject \\
\hline $\mathbf{h}$ & Proposer gets & $\$ 25$ & and Responder gets & $\$ 7$ & Accept & Reject \\
\hline $\mathbf{i}$ & Proposer gets & $\$ 20$ & and Responder gets & $\$ 8$ & Accept & Reject \\
\hline $\mathbf{j}$ & Proposer gets & $\$ 15$ & and Responder gets & $\$ 9$ & Accept & Reject \\
\hline k & Proposer gets & $\$ 10$ & and Responder gets & $\$ 10$ & Accept & Reject \\
\hline 1 & Proposer gets & $\$ 5$ & and Responder gets & $\$ 11$ & Accept & Reject \\
\hline $\mathbf{m}$ & Proposer gets & $\$ 2.50$ & and Responder gets & $\$ 11.50$ & Accept & Reject \\
\hline
\end{tabular}




\section{C.2: Incentivized Beliefs}

\section{ADDITIONAL INSTRUCTIONS}

\section{Procedure}

In this section, you will be asked what do you think is the percent chance that each of the displayed Proposal Rules is Rejected by the Responder. Please provide a number between 0 and 100 - the percentage chance with which you think the Responder will Reject the Proposal Rule.

You will make 12 predictions for different proposal rules in total. One of them will be chosen to determine your payment. Your payment in this round will depend on your decisions and a chance.

\section{Payment}

You will be able to earn up to $\$ \mathbf{1 0}$ in addition to your earnings in the decision stage. You will receive the additional payment if you are able to outperform the random machine in predicting the behavior of other participants in the room. After you submit all your predictions, your earnings will be calculated according to the following procedure:

(1) One of the proposal rules will be chosen at random

(2) One participant from the session (excluding you) will be picked at random and her decision will be retrieved

(3) Your prediction error is computed as ((your prediction)/100 $)^{2}$ if the decision picked by the selected participant is Accept and $(1-\text { (your prediction)/100 })^{2}$ if the decision picked by the selected participant is Reject

(4) A uniform random number will be drawn form the interval from 0 to 1

(5) You will receive $\$ 10$ (in addition to money you already earned) if your prediction error is below random number and will not receive any additional payment otherwise.

\section{Examples}

We now consider some examples.

EXAMPLE: Assume you believe that $50 \%$ of Responders rejected the proposal rule under consideration. We will consider three cases.

Assume your predict that there is a $50 \%$ chance a Responder will Reject the offer.

If the randomly retrieved decision was Reject, your prediction error is $\left(1-\frac{50}{100}\right)^{2}=$ $.5^{2}=.25$. Since the probability a random number is drawn between 0.25 and 1 is $75 \%$, you will get $\$ 10$ with $75 \%$ probability and $\$ 0$ with $25 \%$ probability. If the randomly 
retrieved decision was Accept, your prediction error is $\left(\frac{50}{100}\right)^{2}=.5^{2}=.25$. Since the probability a random number is drawn between 0.25 and 1 is $75 \%$, you will get $\$ 10$ with $75 \%$ probability and $\$ 0$ with $25 \%$ probability. Assuming that with probability .5 the randomly retrieved decision is Reject and with probability .5 is Accept. Hence, your probability of winning the additional prize is $.5 \times .75+.5 \times .75=.75$. That is you will earn extra $\$ 10$ with probability $75 \%$.

Assume instead that you report that with $0 \%$ probability a Responder will Reject the offer. If the randomly retrieved decision was Reject. Hence, your prediction error is $\left(1-\frac{0}{100}\right)^{2}=1^{2}=1$. Since the probability a random number is drawn between 1 and 1 is $0 \%$, you will get $\$ 10$ with $0 \%$ probability and $\$ 0$ with $100 \%$ probability. If the randomly retrieved decision was Accept. Hence, your prediction error is $\left(\frac{0}{100}\right)^{2}=0^{2}=0$. Since the probability a random number is drawn between 0 and 1 is $100 \%$, you will get $\$ 10$ with $100 \%$ probability and $\$ 0$ with $0 \%$ probability. That is you are winning the prize with probability $100 \%$ in this case. Assuming that with probability .5 the randomly retrieved decision is Reject and with probability .5 is Accept. Hence, your probability of winning the additional prize is $.5 \times 0+.5 \times 1=.5$. That is you will earn extra $\$ 10$ with probability $50 \%$. Which is lower than probability of winning if you would report your real expectation $(75 \%)$.

Assume instead that you report that with $100 \%$ probability a Responder will Reject the offer. If the randomly retrieved decision was Reject. Hence, your prediction error is $\left(1-\frac{100}{100}\right)^{2}=0$. Since the probability a random number is drawn between 0.0 and 1 is $100 \%$, you will always get paid is the retrieved decision if Reject. If the randomly retrieved decision was Accept. Hence, your prediction error is $\left(\frac{100}{100}\right)^{2}=1^{2}=1$. In this case you will never get paid is the retrieved decision if Accept. That is you are winning the prize with probability $0 \%$ in this case. Assuming that with probability .5 the randomly retrieved decision is Reject and with probability .5 is Accept. Hence, your probability of winning the additional prize is $.5 \times 1+.5 \times 0=.5$. That is you will earn extra $\$ \mathbf{1 0}$ with probability $50 \%$. Which is lower than probability of winning if you would report your real expectation $(75 \%)$.

As we illustrated above in order to maximize your probability of winning please honestly report the probability of winning which would be as close as possible to the real frequency of rejecting the offer. Since any deviation from honest report will only decrease your probability of winning. 
You can continue now

Good luck! 